\begin{tabular}{|c|c|}
\hline MAY 121997 (21 ENGINERRING DATA TRANSMITTAL & 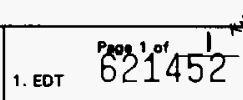 \\
\hline
\end{tabular}

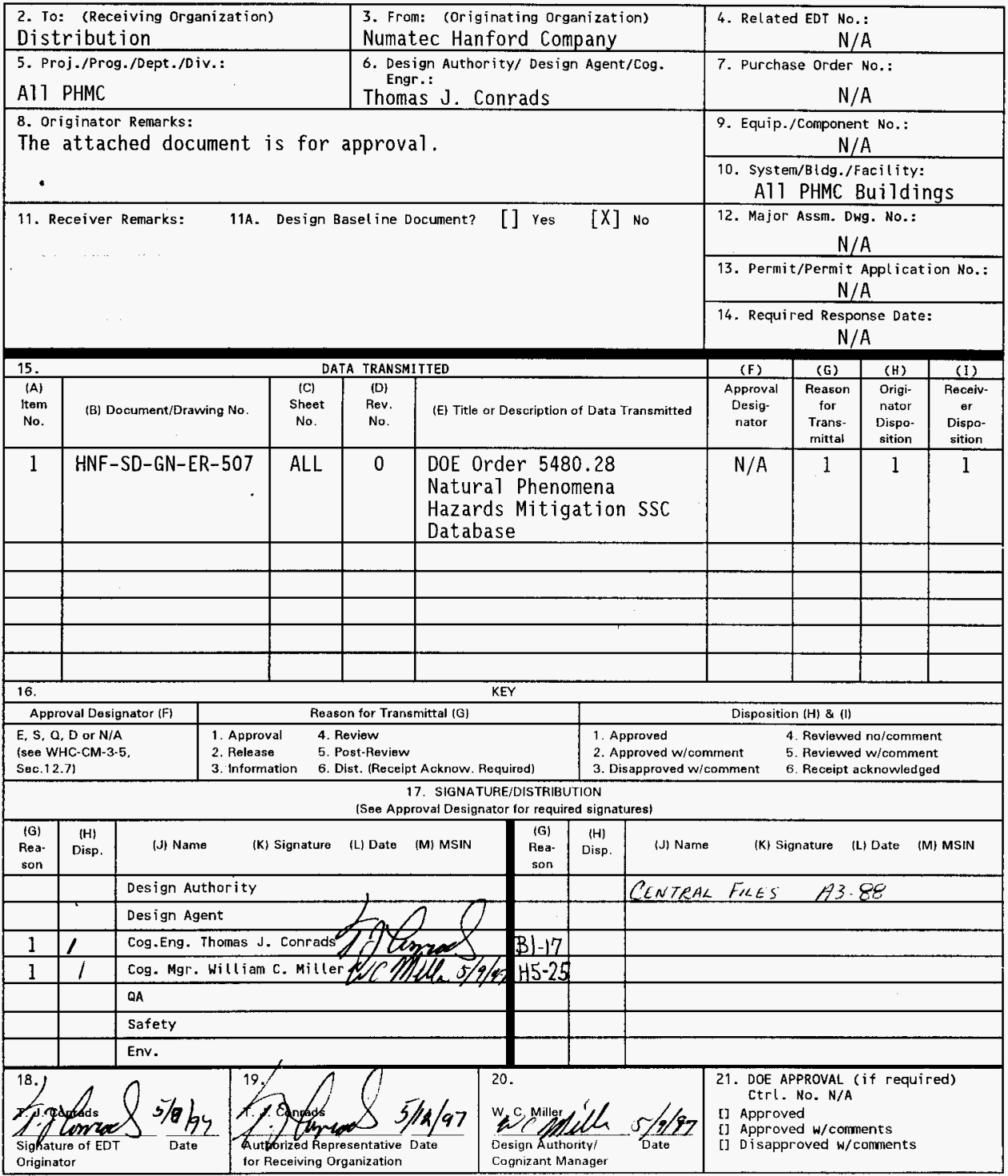




\section{DOE Order 5480.28 Natural Phenomena Hazards Mitigation System, Structure, Component Database}

\section{T. J. Conrads}

Numatec Hanford Company, Richland, WA 99352

U.S. Department of Energy Contract DE-AC06-96RL13200

$\begin{array}{lll}\text { EDT/ECN: } & 621452 & \text { UC: } 607 \\ \text { Org Code: } & 8 C 400 & \text { Charge Code: } \\ \text { B\&R Code: } & \text { YN0100000 } & \text { Tota1 Pages: } 7675 \mathrm{KN}\end{array}$

Key Words: Buildings, Seismic, Natural Phenomena Hazards, DOE Order 5480.28 , Inventory List

Abstract: This document describes the Prioritization Phase Database that was prepared for the Project Hanford Management Contractors to support the implementation of DOE Order 5480.28. Included within this document are three appendices which contain the prioritized list of applicable Project Hanford Management Contractors Systems, Structures, and Components. These appendices include those assets that comply with the requirements of DOE Order 5480.28 , assets for which a waiver will be recommended, and assets requiring additional information before compliance can be ascertained.

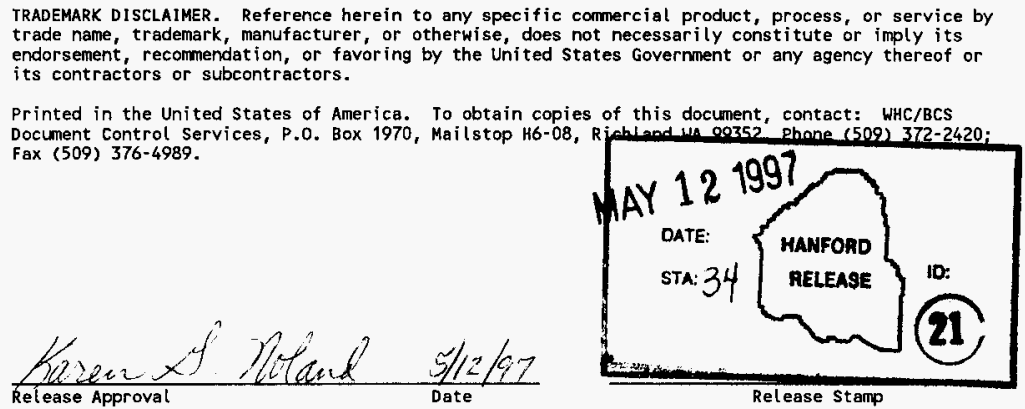




\section{DOE ORDER 5480.28 \\ NATURAL PHENOMENA HAZARD MITIGATION SYSTEM, STRUCTURE, COMPONENT DATABASE \\ PROJECT HANFORD MANAGEMENT CONTRACTORS OWNED AND LEASED SSCS}

HNF-SD-GN-ER-507, Rev. 0

Prepared For:

U.S. Department of Energy

Richland Operations Office

Richland, Washington

Prepared by

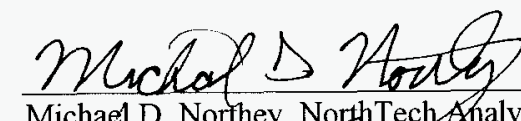

Michael D. Northey, NorthTech Analysis and Design

Approved by

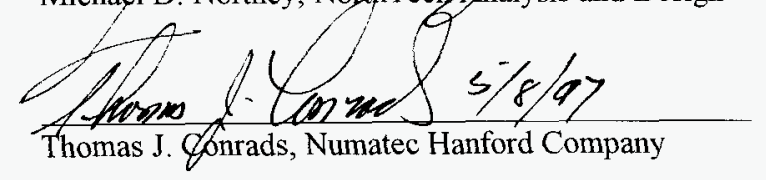




$$
\text { HNF SD-GN-ER-5OH, REV }{ }_{* N}
$$

This page intentionally left blank. 


\section{EXECUTIVE SUMMARY}

The U.S. Department of Energy (DOE) Order 5480.28, Natural Phenomena Hazards Mitigation, requires that the contractors shall establish an implementation plan to structurally evaluate and upgrade existing structures, systems, and components. This plan should incorporate a prioritized schedule for the evaluation of existing structures, systems. and components because it is recognized that there are time, funding, and programmatic mission considerations that preclude near-term compliance for all assets.

Revision 0 of the implementation plan for DOE Order 5480.28, WHC-SP-1175 (WHC 1996), included a database of applicable assets and described the process by which the assets should be prioritized. This document describes the process used to complete the preliminary database, to prioritize the assets, and to categorize them as either acceptable, requiring further action, unacceptable, or as assets that should be exempted from the DOE Order 5480.28 requirements. Included as appendices are the results of this process. These appendices will be included in a subsequent revision of the implementation plan, which will be known as HNF-SP-1175, Revision 1, Fluor Daniel Hanford Implementation Plan for DOE Order 5480.28, Natural Phenomena Hazards Mitigation. 
HNF-SD-GN-ER-507, REV 0

This page intentionally left blank. 


\section{CONTENTS}

1.0 INTRODUCTION $\ldots \ldots \ldots \ldots \ldots \ldots \ldots \ldots \ldots \ldots \ldots \ldots \ldots \ldots \ldots \ldots \ldots$

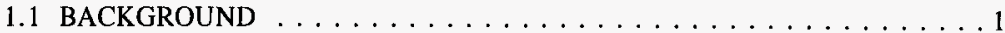

1.2 PRIORITIZATION PROCESS $\ldots \ldots \ldots \ldots \ldots \ldots \ldots \ldots \ldots$

2.0 NATURAL PHENOMENA HAZARDS EVALUATION DATABASE $\ldots \ldots \ldots . .3$

3.0 INHERENTLY RUGGED OR UNIMPORTANT STRUCTURES

ARE LISTED IN APPENDIX A . . . . . . . . . . . . . 8

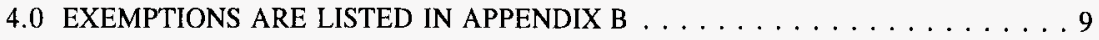

5.0 ASSETS REQUIRING FURTHER EVALUATION ARE LISTED

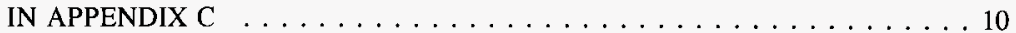

5.1 PERFORMANCE CATEGORIES $\ldots \ldots \ldots \ldots \ldots \ldots \ldots \ldots \ldots$

5.2 OCCUPANT CONSIDERATIONS $\ldots \ldots \ldots \ldots \ldots \ldots \ldots \ldots$

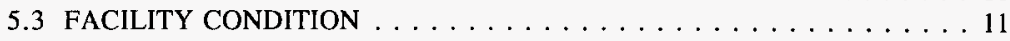

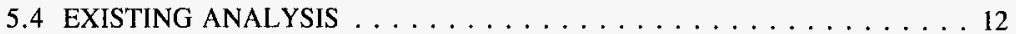

6.0 REFERENCES . . . . . . . . . . . . . . . . . . . . . 14

\section{LIST OF APPENDIXES}

A COMPLIANCE BASELINE FOR NEW AND EXISTING BUILDINGS AND STRUCTURES IN COMPLIANCE WITH DOE ORDER $5480.28 \ldots \ldots \ldots$. A-1

B COMPLIANCE BASELINE FOR EXISTING BUILDINGS AND STRUCTURES WHERE AN EXEMPTION OR DEVIATION

IS BEING REQUESTED $\ldots \ldots \ldots \ldots \ldots \ldots \ldots \ldots$. . . . . . . . .

C COMPLIANCE BASELINE AND PRIORITIZATION SCHEDULE FOR EXISTING BUILDINGS AND STRUCTURES REQUIRING NATURAL

PHENOMENA HAZARDS MITIGATION 


\section{HNF-SD-GN-ER-507, REV 0 \\ LIST OF FIGURES}

1. Natural Phenomena Hazards Prioritization Worksheet $\ldots \ldots \ldots \ldots \ldots$

\section{LIST OF TABLES}

1. Facilities Natural Phenomena Hazard Compliance

Database Structure . . . . . . . . . . . . . . . .4

2 Project Hanford Management Contractors Asset Matrix $\ldots \ldots \ldots \ldots \ldots$ 
HNF-SD-GN-ER-507, REV 0

\section{LIST OF TERMS}

$\begin{array}{ll}\text { ATC } & \text { Applied Technology Council } \\ \text { DOE } & \text { U.S. Department of Energy } \\ \text { FEMA } & \text { Federal Emergency Management Agency } \\ \text { NPH } & \text { natural phenomena hazard } \\ \text { PC } & \text { performance category } \\ \text { PHMC } & \text { Project Hanford Management Contractor } \\ \text { SSCs } & \text { structures, systems, and components } \\ \text { WHC } & \text { Westinghouse Hanford Company }\end{array}$


HNF-SD-GN-ER-507, REV 0

This page intentionally left blank. 


\subsection{INTRODUCTION}

\subsection{BACKGROUND}

The policy of the U.S. Department of Energy (DOE) is to design, construct, and operate facilities so that onsite workers, the public, and the environment are protected from Natural Phenomena Hazards (NPH). The NPH are unexpected acts of nature that may pose a threat or danger to workers, the public, or to the environment. Examples of NPH at the Hanford Site are earthquakes, extreme winds (hurricane and tornado), snow, flooding, volcanic ashfall, and lightning strike.

Fluor Daniel Hanford, Inc. is following a phased and graded approach program for compliance with DOE Order 5480.28, Natural Phenomena Hazards Mitigation. Activities are being accomplished in four phases.

Mobilization. Development of NPH structural design criteria for new and existing facilities, as well as a strategy and plan for implementation

Prioritization. Prioritization of existing facilities and issuance of an implementation plan required by DOE Order 5480.28.

Evaluation. Evaluation of NPH vulnerability of existing facilities not known to be in compliance with DOE Order 5480.28; assurance that designs for new structures, systems, and components (SSCs) and modifications for existing facilities are in compliance

Upgrade. Structural upgrade of non-compliant facilities, if necessary, when justified by a risk-benefit analysis.

The activities and the level of depth, rigor, and thoroughness in accomplishing them are determined by applying a graded approach. The basis for the graded approach is the designation of facilities and structures into one of five performance categories based on safety function, mission, and cost.

The release of implementation plan, WHC-SP-1175 Revision 0, on September 26, 1996 (WHC 1996), and a subsequent approval by the U.S. Department of Energy Richland Operations Office on November 19, 1996, marked completion of the mobilization phase. This document develops the process for the prioritization phase and is used in the development of the prioritized list of Project Hanford Management Contractors' SSCs. That list is included in the appendices attached to this document. All the applicable Project Hanford Management Contractors' (PHMCs) assets and their status of compliance with the requirements of DOE Order 5480.28 are listed in the appendices. 
HNF-SD-GN-ER-507, REV 0

\subsection{PRIORITIZATION PROCESS}

The process of prioritization identifies sites of greatest vulnerability to NPH effects and identifies existing buildings and structures of importance in terms of safety, mission, and cost. These important buildings and structures are then prioritized, based on their importance to safety [i.e. performance category (PC), occupancy considerations, facility condition, and existing structural analysis].

Facilities with low NPH vulnerability because of inherent ruggedness or benign site conditions are eliminated from further consideration, and they are listed in Appendix A. Appendix B contains the list of those facilities and structures that satisfy an exemption criteria. The remaining facilities and structures are included in Appendix C.

Appendix $\mathrm{C}$ lists the facilities and structures by priority, based on grading the parameters mentioned above. At the time this document was prepared, those assets for which only limited information was available are also included in Appendix C. These assets are designated PCy, and many are expected to be moved to the lists in Appendices A or B as information becomes available.

The following sections describe the DOE Order 5480.28 NPH SSC Database and the process by which the appendices were developed using Access ${ }^{1}$ software.

1 Trademark of the Microsoft Corporation. 
HNF-SD-GN-ER-507, REV 0

\subsection{NATURAL PHENOMENA HAZARDS EVALUATION DATABASE}

The NPH survey sheets to compile the information necessary to perform the prioritization of the applicable SSCs were distributed to all PHMC facility managers. The process for compilation of this information is delineated in WHC-SP-1175, Rev. 0 (WHC 1996). A preliminary DOE Order 5480.28 NPH SSC Database is included in WHC 1996. The database included in this document contains updated information that has been accumulated using the NPH survey form since the release of WHC-SP-1175, Rev. 0 .

Table 1 lists the structure and drivers of the NPH database fields that are included for each asset. The fields in the database are completed from the information gathered using the NPH survey sheets and from subsequent evaluations made based on the contents of the asset records. Figure 1 shows a copy of the NPH Prioritization Worksheet (survey form). 


\section{HNF-SD-GN-ER-507, REV 0}

Table 1. Facilities Natural Phenomena Hazard Compliance Database Structure. (2 Sheets)

\begin{tabular}{|c|c|c|c|c|c|}
\hline \multirow{2}{*}{ DATABASE FIELDS } & \multirow{2}{*}{ ENTER } & \multicolumn{3}{|c|}{ FIELD REQUIRED BY } & \multirow{2}{*}{ SOURCE OF DATA } \\
\hline & & PEMA & 5480.28 & Other & \\
\hline $\begin{array}{l}\text { SITE IDENTIFICATION } \\
\text { State } \\
\text { Coutnty } \\
\text { Agency } \\
\text { Site name } \\
\text { Number of facilities }\end{array}$ & $\begin{array}{l}\text { Code } \\
\text { Name } \\
\text { Code } \\
\text { Name } \\
\text { No. } \\
\end{array}$ & $\begin{array}{l}\text { Yes } \\
\text { Yes } \\
\text { Yes } \\
\text { Yes }\end{array}$ & & & \begin{tabular}{|l} 
Constant \\
WA \\
- TBD \\
DOE \\
Hanford Site \\
-- TBD
\end{tabular} \\
\hline $\begin{array}{l}\text { FACILITY IDBNTIFICATION } \\
\text { Area location } \\
\text { Facility number } \\
\text { Facility name }\end{array}$ & $\begin{array}{l}\text { Name } \\
\text { No. } \\
\text { Name }\end{array}$ & $\begin{array}{l}\text { Yes } \\
\text { Yes }\end{array}$ & & $\begin{array}{l}\text { Prioritization } \\
\text { Prioritization } \\
\text { Prioritization }\end{array}$ & $\begin{array}{l}\text { FacilitiesCORE } \\
\text { FacilitiesCORE } \\
\text { FaciliticsCoRE }\end{array}$ \\
\hline $\begin{array}{l}\text { FACILITY CLASSIFICATION } \\
\text { New or Existing } \\
\text { Type: bldg, mobile, etc } \\
\text { Nuclear or Non-nuclear } \\
\text { Occupancy class } \\
\text { Essential } \\
\text { Historic } \\
\text { Status: active, surplus, etc. }\end{array}$ & $\begin{array}{l}\text { N/E } \\
\text { Code } \\
\text { Code } \\
\text { Code } \\
\text { Code } \\
\text { Code } \\
\text { Code }\end{array}$ & $\begin{array}{l}\text { Yes } \\
\text { Yes } \\
\text { Yes }\end{array}$ & $\begin{array}{l}\operatorname{Sec} 10 \\
\sec 3 a \\
\operatorname{Sec} 5 a\end{array}$ & Prioritization & $\begin{array}{l}\text { FacilitiesCORE } \\
\text { IH Safety Fire } \\
\text { FacilitiesCORE }\end{array}$ \\
\hline $\begin{array}{l}\text { OCCUPANT IDENTIFICATION } \\
\text { Owned, leased, other } \\
\text { Responsible contractor } \\
\text { Facility manager } \\
\text { Using programs }\end{array}$ & $\begin{array}{l}\text { Code } \\
\text { Name } \\
\text { Name } \\
\text { Name }\end{array}$ & & $\begin{array}{l}\operatorname{Sec} 4 \\
\operatorname{Sec} 4\end{array}$ & $\begin{array}{l}\text { Accountability } \\
\text { Entry } \\
\text { Funding }\end{array}$ & $\begin{array}{l}\text { FacilitiesCORE } \\
\text { FacilitiesCORE } \\
\text { FacilitiesCORE }\end{array}$ \\
\hline $\begin{array}{l}\text { DESIGN HISTORY } \\
\text { Building code used } \\
\text { Year code issued } \\
\text { Year construction complete } \\
\text { Year last modified } \\
\text { Year last evaluated } \\
\text { Evaluation document number } \\
\text { Margin of safety }\end{array}$ & $\begin{array}{l}\text { Cofte } \\
\text { Year } \\
\text { Year } \\
\text { Year } \\
\text { Year } \\
\text { No. } \\
\text { No. }\end{array}$ & Yes & & $\begin{array}{l}\text { Prioritization } \\
\text { Prioritization } \\
\text { Prioritization } \\
\text { Prioritization } \\
\text { Prioritization } \\
\text { Prioritization } \\
\text { Prioritization }\end{array}$ & FacilitiesCORE \\
\hline $\begin{array}{l}\text { FACILITY DESCRIPTION } \\
\text { Covered floor space } \\
\text { Number of floors } \\
\text { Poundation soil type } \\
\text { Substructure type } \\
\text { Superstructure type }\end{array}$ & $\begin{array}{l}\mathrm{m}^{2} 2 \\
\text { No. } \\
\text { Code } \\
\text { Code } \\
\text { ATC-21 }\end{array}$ & $\begin{array}{l}\text { Yex } \\
\text { Yes } \\
\text { Yes } \\
\text { Yes } \\
\text { Yes }\end{array}$ & & $\begin{array}{l}\text { Prioritization } \\
\text { Prioritization } \\
\text { Prioritization }\end{array}$ & FacilitiesCORE \\
\hline $\begin{array}{l}\text { FACILITY CONDITION } \\
\text { Foundation soil } \\
\text { Substructure } \\
\text { Superstructure } \\
\text { Exterior (non-stiuctural) } \\
\text { Interior (non-structural) } \\
\text { Structural accidents } \\
\text { ATC rating }\end{array}$ & $\begin{array}{l}\text { Code } \\
\text { Code } \\
\text { Code } \\
\text { Code } \\
\text { Code } \\
\text { Code } \\
\text { Scote }\end{array}$ & & & $\begin{array}{l}\text { Prioritization } \\
\text { Prioritization } \\
\text { Prioritization } \\
\text { Prioritization } \\
\text { Prioritization } \\
\text { Prioritization } \\
\text { Prioritization }\end{array}$ & \\
\hline $\begin{array}{l}\text { SAFETY IMPORTANCE } \\
\text { Number of accupants } \\
\text { Number of visitors } \\
\text { Hazardous materials } \\
\text { Hazard category } \\
\text { Hazard class } \\
\text { Safety class } \\
\text { Performance category } \\
\text { FEMA high risk code } \\
\text { SC-1 SSCE } \\
\text { SAR document number }\end{array}$ & $\begin{array}{l}\text { No. } \\
\text { No. } \\
\text { Nane } \\
\text { No. } \\
\text { Code } \\
\text { No. } \\
\text { No. } \\
\text { Code } \\
\text { No. } \\
\text { No. }\end{array}$ & Yes & $\begin{array}{l}\operatorname{Sec} 11 a(5) \\
\operatorname{Sec} 3 \mathrm{a} \\
\operatorname{Sec} 3 \mathrm{a} \\
\operatorname{Sec} 3 \mathrm{a} \\
\operatorname{Sec} 3 \mathrm{a} \\
\operatorname{Sec} 10 \mathrm{~b} \\
\operatorname{Sec} 7 f\end{array}$ & $\begin{array}{l}\text { Prioritization } \\
\text { Prioritization } \\
\text { Prioritization } \\
\text { Order } 5480.23 \\
\text { Order } 5481.1 \mathrm{~B} \\
\text { Prioritization }\end{array}$ & $\begin{array}{l}\text { FacilitiesCORE } \\
\text { SAR } \\
\text { SAR } \\
\text { SAR } \\
\text { SAR } \\
\\
\text { SAR } \\
\text { SAR }\end{array}$ \\
\hline
\end{tabular}


Table 1. Facilities Natural Phenomena Hazard

Compliance Database Structure. (2 Sheets)

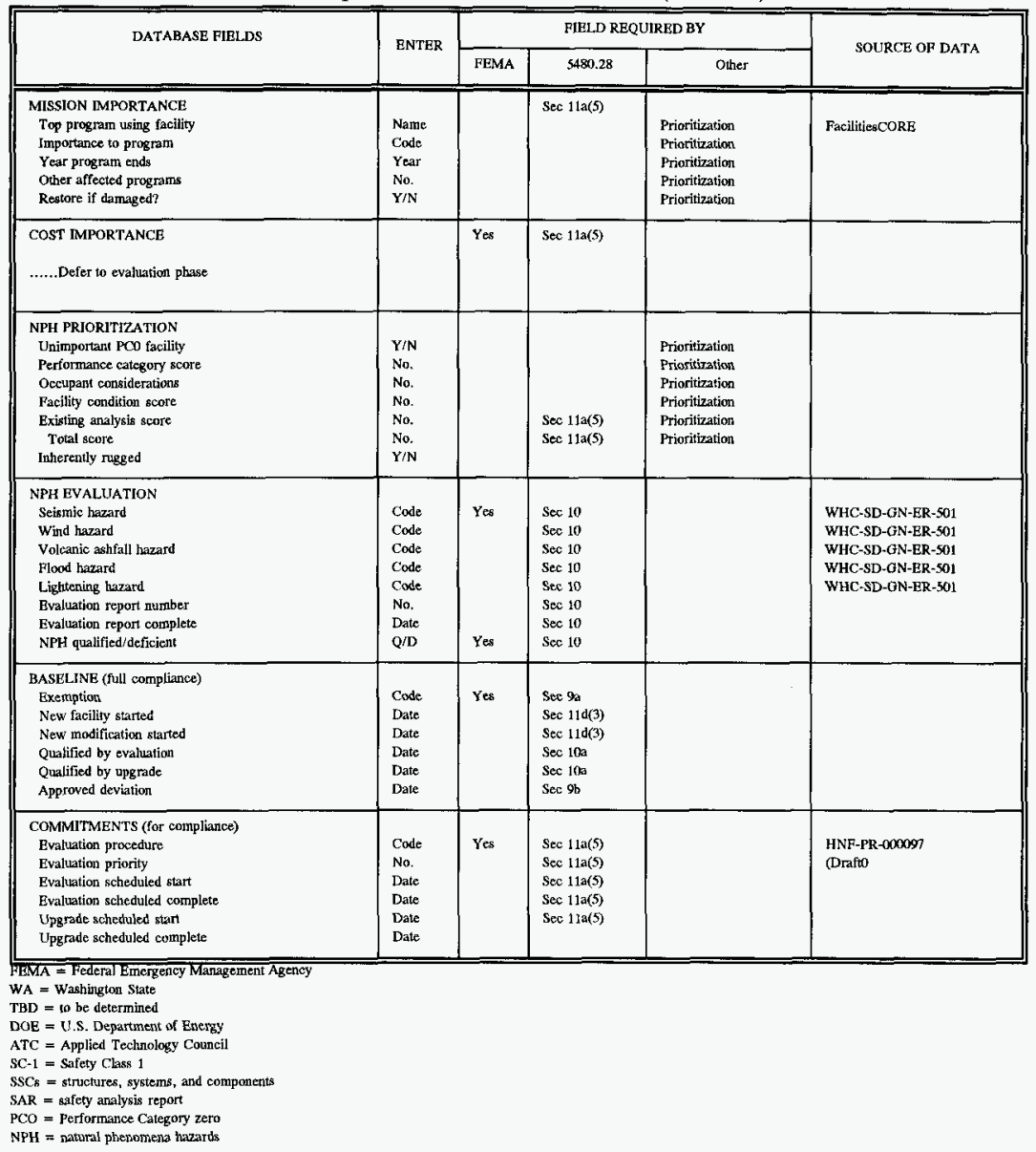

Conrads, T.J., Engineering Design and Evaluations, HNF-PR-000097 (Draft), Numatec Hanford Corporation, Richland, Washington.

Tallman, A.M. 1996, Natural Phenomena Hazards, Hanford Site, South Central, WHC-SD-GN-ER-501, Westingthouse Hanford Company, Richland, Washington. 
Figure 1. Natural Phenomena Hazards Prioritization Worksheet.

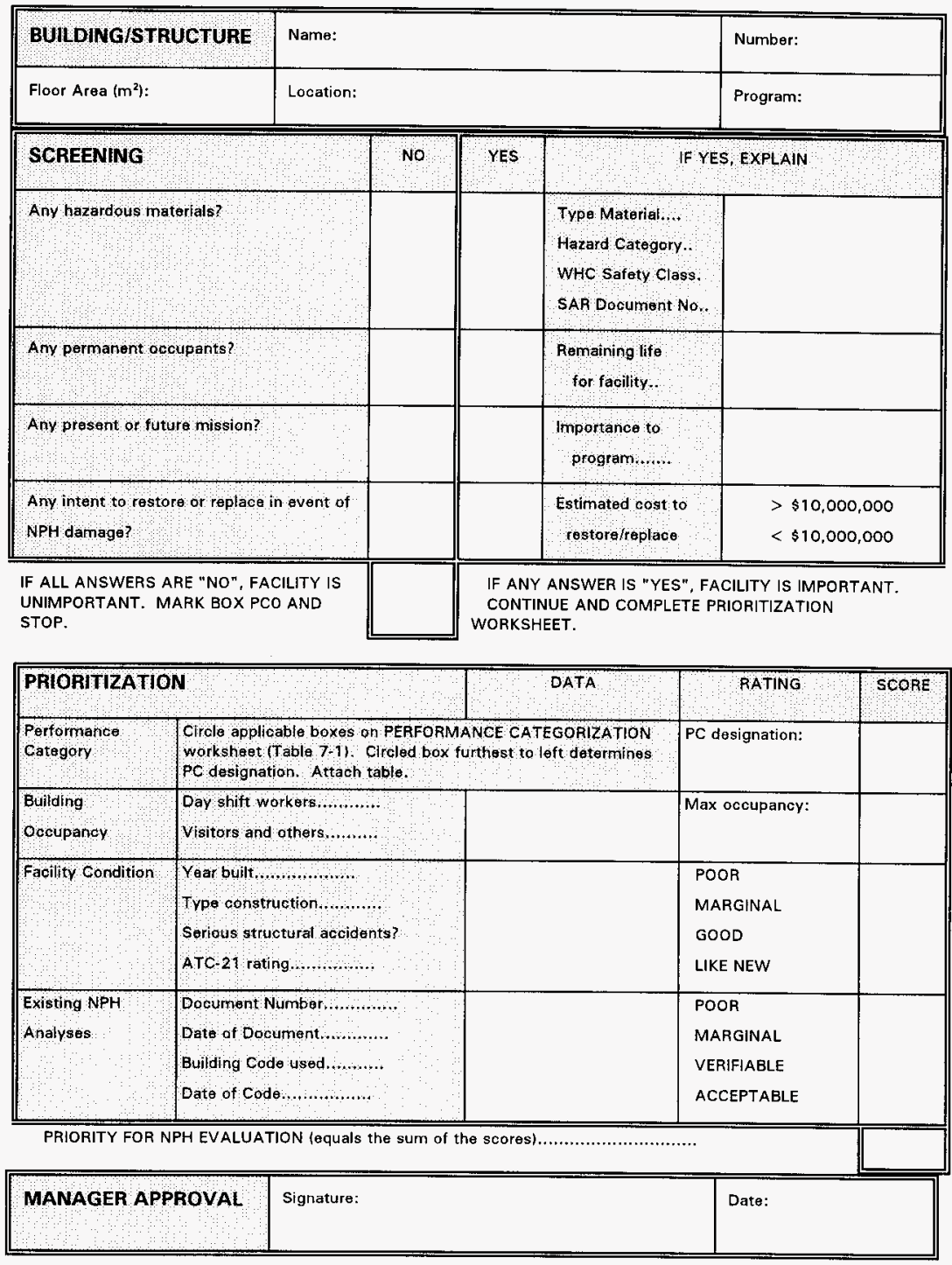


Table 2 shows a matrix of the Project Hanford Management Contractors with a listing of their managed assets as a function of exemption status. The exemption status was determined after information from completed NPH survey sheets had been incorporated into the database. Since Lockheed Martin Hanford Company (LMHC) was not able to return any survey sheets, all LMHC assets were assigned an E0, non-exempt status. At the time of the release of this document, the assignment of the responsible contractor for the 157 assets listed under "Transition Buildings" was under negotiation, and therefore are also assigned a non-exempt status, E0.

Table 2. Project Hanford Management Contractors Asset Matrix.

\begin{tabular}{|c|c|c|c|c|c|c|c|}
\hline $\begin{array}{l}\text { Exempt } \\
\text { Status }\end{array}$ & $\begin{array}{l}\text { Transition } \\
\text { Buildings }\end{array}$ & BWHC & DESH & DYN & LMHC & RFSH & Totals \\
\hline EO* & 182 & 46 & 2 & 57 & 545 & 28 & 860 \\
\hline E1 & & 169 & 20 & 133 & & 47 & 369 \\
\hline E3 & & 44 & 14 & 115 & & 22 & 195 \\
\hline E5 & & 4 & & 38 & & 13 & 55 \\
\hline E8 & & 2 & 5 & 9 & & 1 & 17 \\
\hline Total & 182 & 265 & 41 & 352 & 545 & 111 & 1496 \\
\hline \multicolumn{8}{|c|}{$\begin{array}{l}\text { BWHC - Babcock \& Wilcox Hanford Company } \\
\text { DESH - DUKE Engineering Services } \\
\text { DYN - Dyncorp } \\
\text { LMHC - Lockheed Martin Hanford Corporation } \\
\text { RFSH - RUST Federal Services of Hanford }\end{array}$} \\
\hline
\end{tabular}


HNF-SD-GN-ER-507, REV 0

\subsection{INHERENTLY RUGGED OR UNIMPORTANT STRUCTURES ARE LISTED IN APPENDIX A}

Appendix A is the Compliance Baseline for New and Existing Buildings and Structures Shown to be in Compliance with DOE Order 5480.28.

Appendix A identifies those SSCs shown to be in compliance with requirements of DOE Order 5480.28. As discussed in WHC-SP-1175, Rev. 0 ( WHC 1996, Section 8.2), unimportant facilities are identified as PC0, and inherently rugged facilities are PC1 SSCs with a priority score less than 6 . The process by which the priority score is derived is discussed in WHC-SP-1175 (WHC 1996, Section 5.0), Unimportant facilities designated as PC0 must meet all of the following screening criteria:

- No hazardous materials

- No permanent occupants

- No present mission

- No intent to restore or replace.

Using Access, the DOE Order 5480.28 NPH Evaluation database was queried to list all assets with $\mathrm{PCO}$ in the performance category field. The resulting list was then updated to replace the null value in the "HOW COMPLY" field, shown in Appendix A, to "Unimportant". The database was then queried to list all PC1 assets with a priority score less than 6. The resulting list was then updated to replace the null value in the "HOW COMPLY" field to "Inh. Rugged". An Access report was then designed to generate the hard copy of this information. Appendix A contains that report. The criteria used in the report query was to list all assets with either "Unimportant" or "Inh. Rugged" in the "HOW COMPLY" field of the NPH Evaluation database.

At the end of the prioritization phase, Appendix A identifies only unimportant and inherently rugged SSCs that are eliminated from further consideration by Section 11.a(5) of the DOE Order 5480.28. 
HNF-SD-GN-ER-507, REV 0

\subsection{EXEMPTIONS ARE LISTED IN APPENDIX B}

Appendix B is the Compliance Baseline for Existing Buildings and Structures Where an Exemption or Deviation is Being Requested.

Appendix B lists only facilities that are designated PC-1, that are exempt by Federal Emergency Management Agency (FEMA )criteria, for which a prioritization worksheet is submitted by the program manager or landlord, and that have a priority score greater than 5 . Generally, facilities in Appendix B have low priority scores; consequently, they also have low evaluation priority.

Exemptions are authorizations to exclude something from requirements. Deviations are authorizations to depart from particular requirements, and comply in a more appropriate, specified manner. Deviations are usually granted before-the-fact; when they are granted after-the-fact, they are often referred to as waivers. Exemptions and deviations are requested in accordance with Section 9 of DOE Order 5480.28, and must be approved in writing by DOE.

The FEMA eliminates facilities meeting one or more of the nine exemption criteria from further consideration (see ICSSC RP-4 and Table 5-2 of FEMA-handbook ICSSC TR17). During prioritization, four FEMA exemption criteria were found to be applicable to SSCs at the Hanford Site. Table 1 shows these applicable exemption criteria and the number of SSCs meeting the criteria and for which exemptions are being requested. For each SSC, the FEMA code justifying the exemption request is shown in the second column of Appendix B.

None of the FEMA exemptions are applicable "for buildings which require a seismic performance objective beyond Substantial Life-Safety because of agency mission requirements" (ICSSC RP-4, Section 1.3). Consequently, at the Hanford Site the FEMA exemptions are not applicable to PC-2, PC-3 or PC-4 facilities. Assets with a Performance Category higher than PC-1 are considered essential and are designated $\mathrm{Z1}$ in the ESSENTIAL field in the database. 


\section{HNF-SD-GN-ER-507, REV 0}

\subsection{ASSETS REQUIRING FURTHER EVALUATION ARE LISTED IN APPENDIX C}

Appendix $\mathrm{C}$ is the Compliance Baseline and Prioritized Schedule for Existing Buildings and Structures Requiring NPH Evaluation.

At the end of the prioritization phase, Appendix $C$ contains all existing assets not listed in Appendices A or B. These facilities are ranked by their priority score for those assets that had a completed worksheet, and followed by the assets where insufficient information for prioritization was available.

The priority score is established by using the information returned with the worksheet. The four factors that form the basis of the prioritization are

- Performance Category

- Occupancy Considerations

- Facility Condition

- Existing Analysis.

\subsection{PERFORMANCE CATEGORIES}

DOE Order 5480.28 requires that, for the purposes of NPH design and evaluation, facilities/SSCs be placed in one of five performance categories. Performance categories are established for facilities/SSCs on the basis of their safety, mission, and cost significance. Performance categories are the basis for the graded approach used in this implementation plan, and are discussed in detail in WHC-1175, Rev.0 (WHC 1996).

Performance categorization is considered appropriate for scaling the potential impact of an NPH event on an existing building or structure. Prioritization scores for the potential building/structure hazard are assigned, based on the following performance categories.

\begin{tabular}{cc}
$\begin{array}{c}\text { Performance } \\
\text { Category }\end{array}$ & Score \\
\cline { 2 - 2 } PC4 & 10 \\
PC3 & 5 \\
PC2 & 3 \\
PC1 & 1 \\
PC0 & 0
\end{tabular}

\subsection{OCCUPANT CONSIDERATIONS}

The higher performance categories (PC4, PC3, and PC2) are mostly concerned with the risks to the offsite population and to onsite personnel. For example, PUREX would 
probably be designated as a PC3 building; whereas an office building is typically designated as a $\mathrm{PC} 1$ structure. Occupancy considerations in this prioritization process emphasize the life safety of workers, visitors, and possibly other occupants of a building.

The occupancy loading of a building is representative of the relative risk to building occupants because of structural failure or collapse during or after a NPH event. Occupancy is defined as the number of people continuously occupying the building for more than 2 hours.

Prioritization scores are assigned based on the maximum allowed building occupancy, N. That is, the sum of the normal shift staff plus visitors in conference rooms, auditoriums, etc.

$\begin{array}{cc}\text { Occupancy } N & \frac{\text { Score }}{10} \\ \mathrm{~N}>100 & 5 \\ 10 \leq \mathrm{N} \leq 100 & 1 \\ 1 \leq \mathrm{N}<10 & 0 \\ 0 & \end{array}$

\subsection{FACILITY CONDITION}

Facility condition encompasses the design capacity of the facility for NPH loads and any degradation of the structure or the foundation that may have reduced that capacity. Buildings and structures normally deteriorate with age or are modified to accommodate a new mission. The rate of deterioration is a function of the maintenance program and any unusual historical structural incidents such as accidents, restorations, and modifications. With nominal maintenance and no major structural incident, and for the purpose of prioritization for NPH evaluation, the facility can be assumed to deteriorate as follows.

- First 5 years. Facility condition should be considered like new.

- Age 6-10 years. Facility condition should be considered good.

- Age 11-25 years. Facility condition should be considered marginal.

- After 25 years. Facility condition should be considered poor.

If the facility has experienced a serious structural accident or major modification, the above deterioration schedule may not be conservative. In this case, a field walkdown to determine the facility condition is indicated. A widely recognized methodology has been developed by the Applied Technology Council (ATC) and endorsed by FEMA. This approach is set forth in ATC 1988.

The procedure is a ranking process to develop the relative capacity of structures to prioritize their detailed evaluation or to determine if detailed evaluation is even necessary. A 


\section{HNF-SD-GN-ER-507, REV 0}

primary evaluation criteria under this methodology is based on the type of structure. The ATC-21 (ATC 1988) identifies 12 building categories and the relative seismic resistance of each type.

The historical performance of these classifications of structures is known and includes assessment of degradation mechanisms such as rusting of structural steel frames, mortar degradation in masonry structures, wood rot, etc. This methodology assigns a rating based on the sum of values assigned to evaluation attributes. The higher the ATC rating, the greater the inherent seismic and other NPH resistance.

Prioritization scores for the facility condition are assigned, based on facility age when there has been no serious structural accident. If there has been a serious structural accident, then the prioritization score should be based on the ATC-21 ratings. If that is not feasible, a prioritization score of 10 should be assigned. The schedule for scoring follows.

\begin{tabular}{|c|c|c|c|}
\hline Facility & ATC-21 & Facility & \\
\hline Age (years) & Rating & Condition & $\underline{\text { Score }}$ \\
\hline$>25$ & $<0$ & Poor & 10 \\
\hline $25-11$ & $0-3$ & Marginal & 5 \\
\hline $10-6$ & $>3$ & Good & 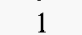 \\
\hline$<5$ & & Like new & 0 \\
\hline
\end{tabular}

\subsection{EXISTING ANALYSIS}

The methodology of NPH evaluation, especially for seismic loads, has evolved significantly over the past 20 years. Consequently, the quality of an evaluation of a structure's capability to adequately resist NPH loads is largely a function of its age. Other important quality considerations are whether the evaluation uses the correct NPH criteria and codes and has been or can be verified. A four-tier classification is used to describe the quality of NPH structural documentation.

- Poor. Documentation has not been found or is older than 20 years. The 20year-old analysis restriction is based on the issue of the 1976 Uniform Building Code (UBC) where unified seismic analysis methodology was promulgated.

- Marginal. Documentation exists and is dated after 1976. Such analyses may or may not comply with NPH requirements found in UCRL-15910.

- Verified. Documentation exists, was performed after 1990, and has been verified by external review. UCRL-15910, issued June 1990, contained the mandatory NPH methodology and a graded approach upon which DOE Order 5480.28 is built. These quality evaluations approach compliance with NPH requirements of UCRL-15910. 
- Acceptable. Documentation exists and is supported by retrievable, verifiable calculations that comply with NPH requirements in UCRL-15910. Such an evaluation was performed to currently acceptable methods, acceptable NPH levels (current seismic response spectra, ANSI or equivalent wind speeds, etc.), and consensus codes (ASME, AISC, ACI, etc.).

Care should be taken that the entire structure and foundation have been adequately evaluated. Where several NPH structural and geotechnical reports exist, the documentation classification should reflect the quality of the least adequate of the necessary evaluations.

The condition of documentation is important mainly for the higher performance categories, PC4, PC3, and PC2. External review (e.g., DOE, Defense Nuclear Facility Safety Board) of NPH designs and evaluations is typically performed only on the higher performance category facilities and SSCs. On the other hand, analyses for PC1 facilities and SSCs can be assumed as acceptable, based on the completion of construction in accordance with the UBC.

Prioritization scores for existing analyses are assigned based on the quality of documentation and on the performance category as follows.

Documentation

$\begin{array}{lclc}\text { Quality } & \underline{\mathrm{PC} 3.4} & \underline{\mathrm{PC} 2} & \underline{\mathrm{PC} 1} \\ \text { Poor } & 10 & 5 & 0 \\ \text { Marginal } & 5 & 3 & 0 \\ \text { Verified } & 1 & 1 & 0 \\ \text { Acceptable } & 0 & 0 & 0\end{array}$

Appendix $\mathrm{C}$ contains those facilities where compliance has not been determined for the following conditions.

(1) Facility records exist in the database for which there is no matching prioritization worksheet. These records were in the various predecessor Hanford Site databases that were compiled to form the present NPH Compliance Database. The unconfirmed assets are believed to include such things as abandoned or demolished buildings, aborted projects, duplicate entries, items transferred to other contractors, and facilities for which the responsible contractor has not submitted a prioritization worksheet.

(2) Data may not be readily available to complete the prioritization worksheet. For example the age of the facility, the building code used, or the existing NPH analysis may not be available to the field.

(3) Prioritization is a screening process and several of the worksheet entries are subjective. Importance to program, number of visitors, facility condition and completeness of existing NPH analysis are examples of subjective data. 
HNF-SD-GN-ER-507, REV 0

\subsection{REFERENCES}

Applied Technology Council, 1988, Rapid Visual Screening of Buildings for Potential Seismic Hazards, ATC-21, ( also issued by the Federal Emergency Management Agency as FEMA 154, July 1988), Applied Technology Council, Redwood City, California.

Conrads, T.J., Engineering Design and Evaluations, HNF-PR-000097 (Draft), Numatec Hanford Corporation, Richland, Washington.

DOE Order 5480.28, 1993, Natural Phenomena Hazards Mitigation, U.S. Department of Energy, Washington, D.C.

DOE, year, Management Plan to Implement E.0. 12941, Seismic Safety of Existing Federally Owned or Leased Buildings, U. S. Department of Energy, Washington, D.C.

ICSSC RP-4, 1994, "Standards of Seismic Safety for Existing Federally Owned or Leased Buildings," Interagency Committee On Seismic Safety in Construction, Gaithersburg, Maryland.

ICSSC TR-17, 1995, How to Suggestions for Implementing Executive Order I294I on Seismic Safety of Existing Federal Buildings, A Handbook, Interagency Committee On Seismic Safety in Construction, Gaithersburg, Maryland.

UBC, 1976, Uniform Building Code, International Conference of Building Officials, Whittier, California.

UCRL-15910, 1990, Design and Evaluation Guidelines for DOE Facilities Subjected to Natural Phenomena Hazards (superseded by DOE Order 5480.28), University of California Lawrence Radiation Laboratory, Livermore, California.

Tallman, A.M. 1996, Natural Phenomena Hazards, Hanford Site, South Central, WHC-SD-GN-ER-501, Westingthouse Hanford Company, Richland, Washington.

WHC 1996, Westinghouse Hanford Company Implementation Plan for U.S. Department of Energy (DOE) Order 5480.28, Natural Phenomena Hazards Mitigation, WHC-SP-1175, Revision 0, Westinghouse Hanford Company. 
HNF-SD-GN-ER-507, REV 0

APPENDIX A

COMPLIANCE BASELINE FOR NEW AND EXISTING

BUILDINGS AND STRUCTURES IN COMPLIANCE

WITH DOE ORDER 5480.28 
HNF-SD-GN-ER-507, REV 0

This page intentionally left blank. 
APPENDIX A. COMPLIANCE BASELINE FOR NEW AND EXISTING BUILDINGS AND STRUCTURES IN COMPLIANCE WITH DOE ORDER 5480.28. TOTAL ASSETS $=326$

\begin{tabular}{|c|c|c|c|c|c|c|c|c|c|c|c|c|}
\hline $\begin{array}{l}\text { WHEN } \\
\text { COMPLY }\end{array}$ & $\begin{array}{l}\text { HOW } \\
\text { COMPLY }\end{array}$ & IDENTIFIER & DESCRIPTON & CONTR & PROJECT & $\begin{array}{l}\text { YEAR } \\
\text { BUILT }\end{array}$ & $\begin{array}{l}\text { YEAR } \\
\text { SURPLUS }\end{array}$ & $\begin{array}{l}\text { AREA } \\
\text { SQA } \\
\text { METER }\end{array}$ & $\begin{array}{l}\text { PERF. } \\
\text { CAT. }\end{array}$ & $\begin{array}{l}\text { TOTAI } \\
\text { OCCUP }\end{array}$ & $\begin{array}{l}\text { FACIUTY } \\
\text { CONDITION } \\
\text { RATING }\end{array}$ & $\begin{array}{l}\text { EXISTING } \\
\text { ANALYSIS } \\
\text { RATTNG }\end{array}$ \\
\hline 15-May-97 & Inh. Rugged & 1161 & NITROGEN BOTTLE STORAGE & $\overline{\text { DYN }}$ & $\overline{G P F}$ & 1987 & & 45 & $\overline{\mathrm{PC} 1}$ & $\overline{2}$ & $\overline{\mathrm{GOOD}}$ & $\overline{\text { POOR }}$ \\
\hline 15-Mny-97 & Inh. Rugged & 1162 & FLAMMABLE STORAGE & DYN & $\overline{G P F}$ & 1987 & 2017 & 827 & PC1 & 8 & GOOD & POOR \\
\hline $15+$ May-97 & Inh. Rugged & 1164 & METAIS BUILDKG & DYN & GPF & 1987 & & 186 & PC1 & 2 & GOOOD & FOOR \\
\hline 15-May-97 & Unimportant & 1170 & BUS TERMINAL & DYN & GPF & & & 6416 & PCO & 0 & & \\
\hline 15-Mny-97 & Inh. Rugged & $1172 \mathrm{~A}$ & SERVICE STATTON & DYN & GPF & 1981 & 2012 & 28 & PCi & 0 & GOOD & ACCEPTABLE \\
\hline 15-May-97 & Unimportant & 1173 & MOTOR STORES BLDG & $\overline{D Y N}$ & GPF & & & 3216 & $\mathrm{PCO}$ & $\overline{0}$ & & \\
\hline $15-$ May-97 & Unimportant & 1174 & BULK PETROLEUM STORAGE FACIUTY & DYN & GPF & & & 2275 & PCO & 0 & & \\
\hline 15-May-91 & Unimportant & 1175 & STORAGE & DYN & GPF & & & 220 & PCO & 0 & & \\
\hline 15-May-97 & Unimportant & 1176 & TIRE STORAGE BUILDING & $\overline{D Y N}$ & GPF & & & 73 & PCO & 0 & & \\
\hline 15-May-97 & Unimportant & 1177 & STTORAGE BŪLDTNGG & DYN & GPF & $?$ & 2002 & 71 & $\mathrm{PCO}$ & 0 & POOR & POOR \\
\hline 15-May-97 & Unimportent & 1179 & ROAD CREW STOKORAGE & DYN & Glp: & & & 93 & PCO & 0 & & \\
\hline 15-May-97 & Inh. Kugged & $119 \mathrm{KE}$ & EXHAUST AIR SAMPLE BUILDING & DESH & SNF & 1755 & & 98 & FCI & 0 & GOOD & POOR \\
\hline 15. May-97 & Inh. Rugged & $1614 \mathrm{KE}$ & ENVIRONMENTAL MONITORING STATTON & DESH & SNF & 1955 & & 6 & PC1 & 0 & GOOD & $\overline{\mathrm{POOOR}}$ \\
\hline 15-May-97 & Inh. Ruggod & $165 \mathrm{KE}$ & COLUMBIA RIVER MONTTORING & DESH & SNF & 1955 & 2002 & 2455 & PCl & $\bar{q}$ & GOOD & POOR \\
\hline 15-May-97 & Inh. Rugged & $165 \overline{K W}$ & POWER CONTROL BUILDING & DESH & SNF & 1955 & 2002 & 510 & $\overline{\mathrm{PCI}}$ & 0 & GOOD & POOR \\
\hline $15-$ May-97 & Inh. kugged & 166AKE & MATERTAL STORAGE BUILDING & DESH & SNF & 1964 & & 27 & PC1 & 0 & GOOD & POOK \\
\hline \begin{tabular}{|l|} 
15-May-97 \\
\end{tabular} & \begin{tabular}{|l|} 
Unimportant \\
\end{tabular} & $1705 \mathrm{KE}$ & EFFLUENT WATER TREATMENT PILOT PLANT & DESH & SNF & & & 130 & PCO & 0 & & \\
\hline 15-May-97 & Inh. Rugged & 1706KEL & DEVELOPMENT LABOORATORY COOLANT SYSTEM & DESH & SNF & 1964 & & 255 & $\mathrm{PCI}$ & 0 & GOOD & POOR \\
\hline 15-May-97 & Inh. Ruggod & 1713KE & SHOP BUILDING AREA & DESH & SNF & $?$ & & 18 & PC1 & 0 & GOOD & $\overline{\mathrm{P} O \overline{O R}}$ \\
\hline $75-M a y-97$ & Unimportant & $1713 \mathrm{~kW}$ & WARAHOUSE & DESH & SNF & & & 74 & FCO & 0 & & \\
\hline 15-May-97 & Inh. Rugged & $1714 \mathrm{KE}$ & OIL AND PAINT STORAGE SHED & DESH & SNF & & & 76 & PC1 & 0 & GOOD & POOR \\
\hline $15-M_{n y-97}$ & Unimportant & $1714 \mathrm{KW}$ & OILPAINT STORAGE & DESH & SNF & & & 19 & $\mathrm{PCO}$ & 0 & & \\
\hline 15-May-97 & Inh. Ruggad & 18IKE & RIVER PUMPHOUSE & DESH & SNF & 1955 & & 393 & $\mathrm{PCl}$ & 0 & GOOD & POOR \\
\hline 15-May-97 & Inh. Rugged & $182 \mathrm{~B}$ & PUMF HOUSE BUILDING & DYN & GPF & 1960 & 2001 & 1365 & PC1 & 6 & GODD & POOR \\
\hline 15-May-97 & Inh. Rugged & $182 \mathrm{D}$ & PUMP HOUSE BUILDING & DYN & GPF & 1960 & 2000 & 1365 & PCl & 6 & GOOD & POOR \\
\hline 15-May-97 & Inh. Rugged & 183-2KE & BASINS/SEDIMENTATKON & DESH & SNF & 1955 & & 21015 & $\overline{\mathrm{PCI}}$ & 0 & GOOD & POOR \\
\hline 15-May-97 & Inh. Kugged & $183-3 \mathrm{KE}$ & BASIN/FILTERS & DESH & SNF & 1955 & & 6073 & PCI & $\overline{0}$ & GOOD & POOK \\
\hline 15-May-97 & Inh. Rugged & $183.1 \mathrm{KE}$ & HEADHOUSE/CKLORIVE & DESH & SNF & 1955 & 2002 & 1075 & PCI & 9 & GODD & POOK \\
\hline 15-Mey-97 & Inh. Ruggod & $183.1 \mathrm{KW}$ & HEADHOUSE/CHLORINE & DESH & SNF & 1955 & & 309 & PC1 & 0 & GOOD & POOR \\
\hline 15-May-97 & Int. Rugged & $183,5 \mathrm{KE}$ & LIME FGEDER BUILDING & DESH & $\overline{\text { SNF }}$ & 1955 & & 98 & PC1 & 0 & GOOD & POOR \\
\hline 15-Mny-977 & Inh. Rugged & $183,6 \mathrm{KE}$ & LYME FEEDER BUILDING & DESH & SNF & 1955 & & 98 & PCI & $\overline{0}$ & GOOD & POOR \\
\hline 15-May-97 & Inh. Kugged & $183 B$ & TRANSFORUER SUBSTATION & DYN & GPF & 1960 & & 6052 & PCI & 3 & GODD & FOOR \\
\hline 15-May-97 & Inh. Rugged & $1908 \mathrm{~K}$ & OUTFALL STRUCTURE & DESH & SNF & 1955 & & 14 & PC1 & 0 & GOOD & POOR \\
\hline 15-Mny-97 & Inh. Rugged & I908KE & EFFLUENT WATER MONTTORING STATION & DESH & SNF & 1955 & & $1 \overline{3}$ & $\overline{\mathrm{P}} \overline{\mathrm{Cl}}$ & 0 & GOOD & POOR \\
\hline 15-May-97 & Unimportant & $190 \mathrm{~kW}$ & HIGH B̈AY STORAGE AREA & DESH & SNF & & & 1269 & $\mathrm{PCO}$ & 0 & & \\
\hline 15-Mny-97 & Thb. Rugged & $2025 \mathrm{EC}$ & EIF TRUCK LOAD-IN FACILITY & RFSH & DEF & 1995 & & & FCI & 5 & IKE NEW & POOK \\
\hline
\end{tabular}




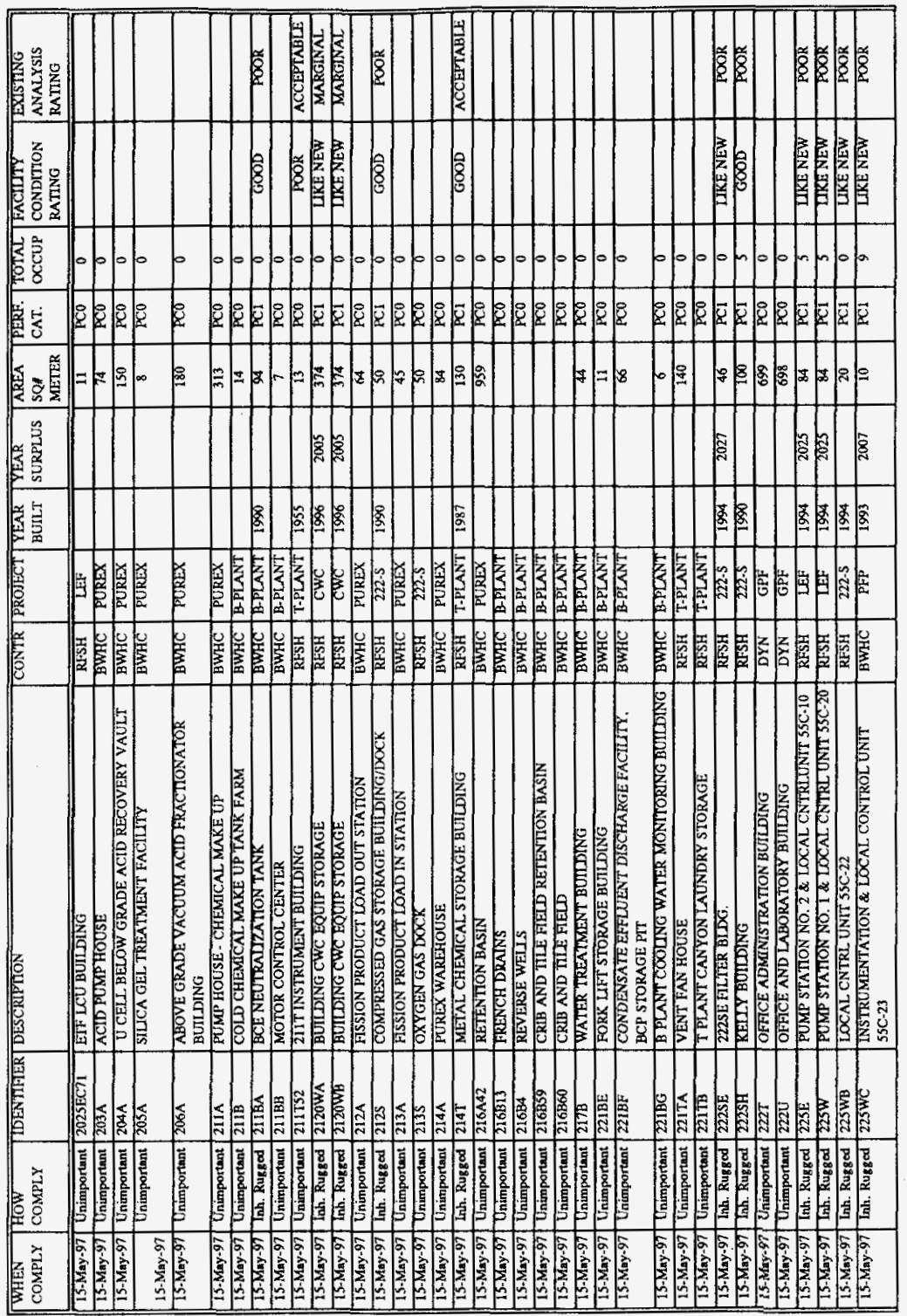




\begin{tabular}{|c|c|c|c|c|c|c|c|c|c|c|c|c|}
\hline $\begin{array}{l}\text { WHEN } \\
\text { COMPLY }\end{array}$ & $\begin{array}{l}\text { HOW } \\
\text { COMPLY }\end{array}$ & TIDENTIFER & DESCRIYTTON & CONTR & PROAECT & $\begin{array}{l}\text { YEAR } \\
\text { BUILT }\end{array}$ & $\begin{array}{l}\text { YEAR } \\
\text { SURPLUS }\end{array}$ & $\begin{array}{l}\text { AREA } \\
\text { SQM } \\
\text { METER }\end{array}$ & $\begin{array}{l}\text { PERF. } \\
\text { CAT. }\end{array}$ & $\begin{array}{l}\text { TOTAL } \\
\text { OCCUP }\end{array}$ & $\begin{array}{l}\text { FACIDTYY } \\
\text { CONDITION } \\
\text { RATING } \\
\end{array}$ & $\begin{array}{l}\text { EXISTING } \\
\text { ANALYSIS } \\
\text { RATING } \\
\end{array}$ \\
\hline 15-May-97 & Unimportant & $234 \mathrm{ZC}$ & STOKAGE BUILDING & BWHC & PFP & & & 10 & $\overline{\mathrm{PCO}}$ & $\overline{0}$ & & \\
\hline 15-May-97 & Unimportant & 2412361 & SETIUNG TANK & BWHC- & PFP & & & & FOO & 0 & & \\
\hline 15-May-97 & Unimportant & $2412 \mathrm{~B}$ & $\begin{array}{l}\text { OPEN AREA SLAB STRUCTURE, URANIUM } \\
\text { STORAGE, PFP COOLING TOWERS }\end{array}$ & BWHC & PFP & & & 100 & $\mathrm{PCO}$ & 0 & & \\
\hline 15-May-97 & Unimportant & $2412 \mathrm{G}$ & 241ZG CHANGE FACILITY & BWHC & PFP & & & 20 & $\mathrm{PCO}$ & 0 & & \\
\hline 15-May-97 & Unimportant & 241ZRB & WASTE WATER RETENTION BASIN & BWHC & PFP & & & & PCO & 0 & & \\
\hline 15-May-97 & Inh. Rugged & $242 A 81$ & WATER SERVICE BUILDING & RFSH & $242 \mathrm{~A}$ & 1989 & & 52 & $\mathrm{PCl}$ & 0 & GOOD & VERIFIED \\
\hline 15-May-97 & Unimportant & 242ALII & LERF STORAGE BUILDING & RTSH & LEF & & & 46 & PCO & $\overline{0}$ & & \\
\hline 15-May-97 & Unimportant & 242AL42 & PUREX PDD/ASD NO 2 STEAM RETENTION BASIN $\# 2$ & RFSH & & & & & $\mathrm{PC0}$ & $\overline{0}$ & & \\
\hline 15.Mny-91 & Unimportant & $242 \mathrm{ALA3}$ & 242A EVAP NO 1STEAM RETENTION BASIN II & RFSH & LEF & & & & PCO & 0 & & \\
\hline 15-Mey-97 & Unimportant & 242ALA4 & 242 A EVAP NO 2 STEAM RETENTION BASIN 12 & RFSH & LEF & & & & $\mathrm{PC} 0$ & 0 & & \\
\hline 15-May-97 & Inh. Rusged & $242 Z \mathrm{~A}$ & MONITORING BUILDING & BWHC & PFP & 1989 & 2007 & 40 & PCI & $\overline{9}$ & GOOD & FOOR \\
\hline 15-Mny-97 & Tnh. Ruggad & $2432 A$ & LOW LEVEL WASTE STORAGE & BWHC & PFP & 1992 & 2007 & 80 & PCI & 9 & UKE NEW & $\overline{\mathrm{POOR}}$ \\
\hline 15-May-97 & Inh. Rugged & $243 \mathrm{ZB}$ & COOLNG TOWERS AND CONCRETE PAD & BWHC & PFP & 1993 & & 20 & PCI & 0 & DKE NEW & VERIFIABLE \\
\hline 15-May-97 & Unimportant & $252 \mathrm{~A}$ & $\begin{array}{l}\text { ELECTRICAL SWITCHING STATION 13.8KV, } \\
\text { STRUCTURE }\end{array}$ & BWFC & PUREX & & & & $\overline{\mathrm{PCO}}$ & 0 & & \\
\hline 15-May-97 & Unimportant & $2701 \mathrm{AB}$ & PUREX BADGE HOUSE & BWHC & PUREX & & & 220 & $\overline{P C 0}$ & 0 & & \\
\hline 15-May-97 & Unimportant & $2701 \mathrm{EC}$ & GUARDSTATON FOR 209E BADGE HOUSE/209-E & DYN & GPF & & & 12 & PCO & 0 & & \\
\hline 15-May-97 & Unimportunt & $2701 \mathrm{M}$ & OFFCE BUILDING & DYN & GPF & & & 29 & $\overline{\mathrm{PCO}}$ & 0 & & \\
\hline 15-Mny-97 & Unimportant & $2701 \bar{Z}$ & ODD BADGE HOUSE (LAY-AWAY STATUS) & BWHC & PFP & & & & $\overline{\mathrm{PCO}}$ & 0 & & \\
\hline 15-May-97 & Unimportant & $27012 B$ & FXCLUSION AREA BADGE HOUSE & BWHC & PFP & & & 13 & $\overline{P C 0}$ & 0 & & \\
\hline 15-May-97 & Unimportant & $2704 \mathrm{C}$ & OFFCE BUIDDING & DYN & GPF & & & 98 & $\mathrm{PCO}$ & 0 & & \\
\hline 15-May-97 & Inh. Rugged & $2706 \mathrm{TA}$ & BUILDING EQUTPMENT DECONTAM & RFSH & T-PLANT & 1993 & & & $\mathrm{PCl}$ & $\overline{\mathbf{0}}$ & DKE NEW & ACCEPTABLE \\
\hline 15-May-97 & Unimportant & $2706 \mathrm{Z}$ & SEPTTC TANK & BWHC & PFP & & & & $\mathrm{PCO}$ & 0 & & \\
\hline 15-May-97 & Unimportant & $2707 E$ & CHANGE HOUSE & $\overline{\mathrm{DYN}}$ & GPF & & & 307 & PCO & $\mathbf{0}$ & & \\
\hline 15-May-97 & Unimportant & $2709 \mathrm{~A}$ & POLEX RAILROAD CUT CHANGE HOUSE & BWHC & POREX & & & 16 & PCO & 0 & & \\
\hline 15-May-97 & Unimportant & $2709 \mathrm{~W}$ & OFFICE BUILDING & DYN & GPF & & & 170 & PCO & 0 & & \\
\hline 15-Mny-97 & Unimportant & 2700 & CONDENSATE COLIECTION TANK & BWHC & B-PLANT & & & & $\mathrm{PCO}$ & 0 & & \\
\hline 15-May-97 & Onimportant & $2710 \mathrm{~W}$ & METAL BUIL DING & DYN & GPF & & & 16 & $\mathrm{PC} 0$ & 0 & & \\
\hline $15-M a y-97$ & Unimportant & $2711 \mathrm{~A}$ & AIR COMPRESSOR BUTLDING & BWHC & PUREX & & & 32 & PCo & 0 & & \\
\hline 15-May-97 & Unimportant & $2711 \mathrm{~B}$ & BREATHING AIR COMPRESSOR BUILDING & BWHC & \begin{tabular}{|l|} 
B-PLANT \\
\end{tabular} & & & 10 & $\mathrm{PC} 0$ & $\overline{0}$ & & \\
\hline 15-May-97| & Inh. Kuggod & 2711EA & REGULATED EQUTPMENT SHOP & DYN & GPF & 1991 & 2022 & 3804 & $\overline{\mathrm{PCI}}$ & 5 & UKKE NEW & ACCËPTABLE \\
\hline $\begin{array}{c}\text { V15-May- } \\
97\end{array}$ & Inh. Rugged & $2711 \mathrm{~EB}$ & HEAVY MÖBIL MAINTENANCE SHOP & DYN & GPF & 1995 & 2027 & 4494 & $\overline{P C 1}$ & 5 & IIKE NEW & ACCEPTABLE \\
\hline 15-May-97 & Unimportant & $2712 \mathrm{~A}$ & PUMPHOUSE AIR & BWHC & PUREX & & & 32 & PCO & 0 & & \\
\hline 15-May-97 & Inh. Rugged & $2712 \mathrm{~T}$ & EIECTRICAL INSTRUMENTATON BUILDING & RFSH & T-PLANT & 1988 & & 6 & $\overline{\mathrm{PCI}}$ & 0 & GOOD & ACCEPTABIF \\
\hline 15-May-97 & Unimportant & $2713 \mathrm{E}$ & OFHCE AD̈MINISTRATTON BUILDING & DYN & GPF & & & 384 & $\mathrm{PCO}$ & 0 & & \\
\hline $15 \cdot \operatorname{Mng}^{-9} 97$ & Onimportant & $2714 \mathrm{~A}$ & DRY CHEMCAL WARMHOUSE STORAGE BUILDING & BWHC & PUKEX & & & 270 & FCo & 0 & & \\
\hline 15-May-97 & Unimportant & $2714 \mathrm{E}$ & & BWHC & PUREX & & & & $\mathrm{PCO}$ & 0 & & \\
\hline 15-May-97 & Unimportant & $2715 \mathrm{E}$ & BUTLDDING OIL AND PAINT STORAGE & DYN & GPF & & & 89 & $\overline{P C O}$ & 0 & & \\
\hline $15-\mathrm{May}-97$ & Unimportant & $2715 \mathrm{M}$ & PAINT STORAGE BUILDING & DYN & GPF & & & 36 & $\overline{P C O}$ & 0 & & \\
\hline
\end{tabular}


4

HNF-SD-GN-ER-507, REV 0

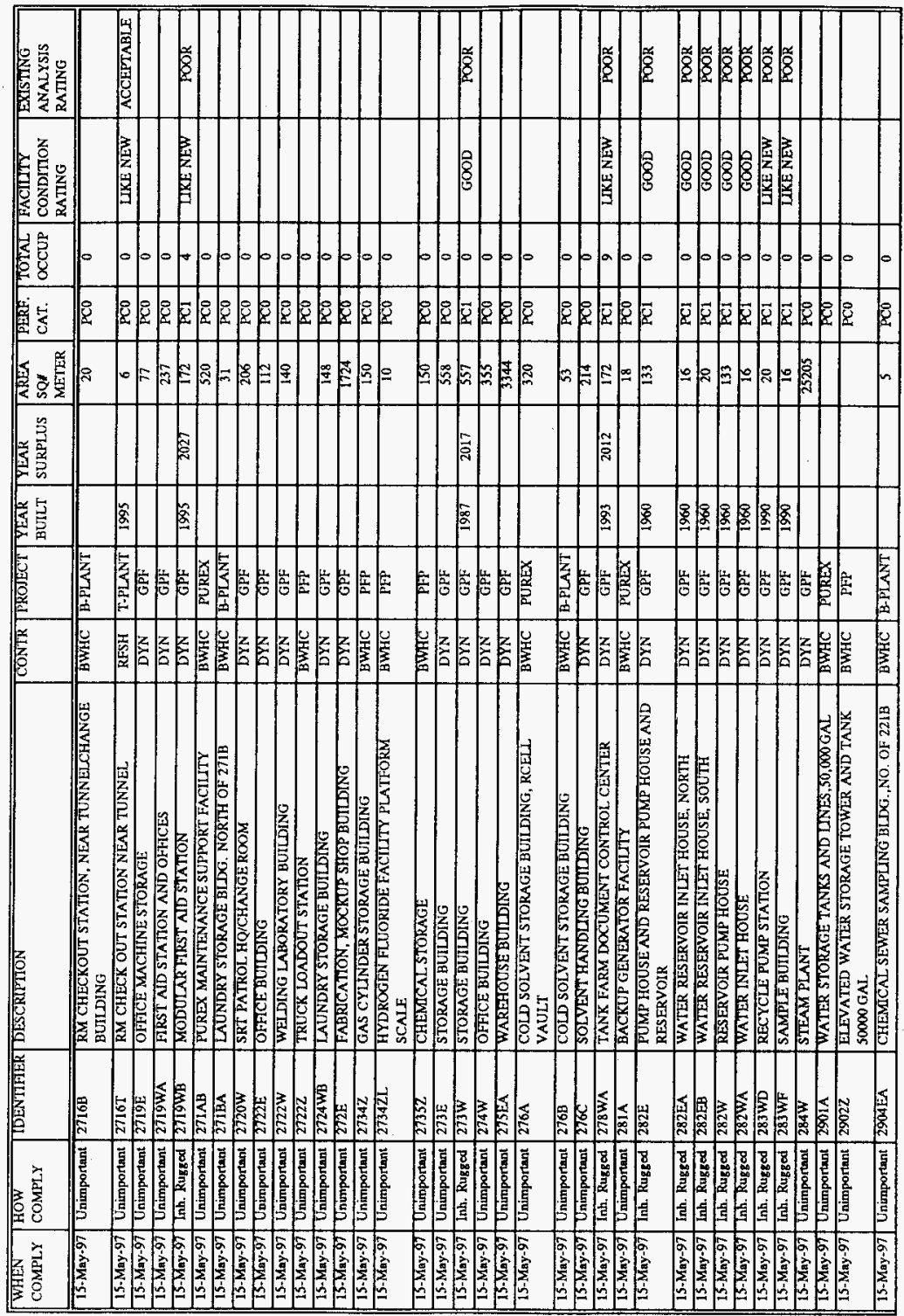




\begin{tabular}{|c|c|c|c|c|c|c|c|c|c|c|c|c|}
\hline $\begin{array}{l}\text { WHES } \\
\text { COMPLY }\end{array}$ & $\begin{array}{l}\text { HOW } \\
\text { COMPLY }\end{array}$ & IDENTIFIR & DESCRPTION & TCONTK & PROJECT & $\begin{array}{l}\text { PEAK } \\
\text { BUILT }\end{array}$ & $\begin{array}{l}\text { YEAAR } \\
\text { SURPLUS }\end{array}$ & \begin{tabular}{|l|} 
AREA \\
SQA \\
METER
\end{tabular} & $\begin{array}{l}\text { PEERF. } \\
\text { CAT. }\end{array}$ & $\begin{array}{l}\text { TOTAL } \\
\text { OCCUP }\end{array}$ & $\begin{array}{l}\text { FACILTY } \\
\text { CONDITION } \\
\text { RATING }\end{array}$ & \begin{tabular}{|l} 
EXISTING \\
ANALYSIS \\
RATING
\end{tabular} \\
\hline 15-Mny-97 & Unimportant & $2904 \mathrm{ZA}$ & RADLATION AND FLOW MONTOORING STATION & BWHC & $\overline{P F P}$ & & & 10 & PCo & $\overline{\overline{0}}$ & & \\
\hline 15-May-97 & Unimportint & $2904 Z \mathrm{ZB}$ & MONTTORING BUIDOING & BWHC & PFP & & & 10 & PCO & 0 & & \\
\hline 15-Mny-97 & Unimportant & $291 \mathrm{~A} 1$ & STACX, 202A MAIN & BWHC & PUREX & & & 17 & $\mathrm{PCO}$ & 0 & & \\
\hline 15-May-97 & Uniuportant & $291 \mathrm{AB}$ & EXYAAUST AIR SAMPLER HOUSE & BWHC & PUREX & & & 6 & $\overline{\mathrm{PCO}}$ & 0 & & \\
\hline 15-May-97 & Unimportant & $29 \mathrm{IAC}$ & EXXAUST AIR SAMPLER HOUSE & BWHC & PUREX & & & 6 & PCO & 0 & & \\
\hline 15.May-97 & Unimportent & 29 IAG & SAMPLE STATIONTH2 & BWLEC & PUREX & & & 10 & PCO & 0 & & \\
\hline 15-May-97 & Unimportant & $29[\mathrm{~A} J$ & SAMPLE STATTON $* 3$ & BWHC & PUREX & & & 8 & $\overline{\mathrm{PCO}}$ & 0 & & \\
\hline 15-May-97 & Unimportant & 29 IAK & TUNNEL SPRAY ENCLOSUURE AND CAISSONS & BWHC & PUREX & & & 5 & $\overline{\mathrm{PCO}}$ & 0 & & \\
\hline 15-May-97 & Unimportant & 291AN & AMMONIA OFFGAS FILTER BLDG & BWHC & PUREX & & & 24 & $\overline{\mathrm{PCO}}$ & 0 & & \\
\hline 15-May-97 & Unimportant & 29IAR & EXHAUST AIR RITER STACK BUILDING & BWHC & PUREX & & & 62 & FCO & 0 & & \\
\hline 15.May-97 & Unimportant & $291 \mathrm{BG}$ & D FLTER INSTRUMENT BLDG & BWHC & B-PLANT & & & 9 & PCO & $\overline{0}$ & & \\
\hline $15-\mathrm{May}-97$ & Unimportant & 2918 & B-PLANT 'F" FILTER & BWHC & B-PLANT & & & 9 & PCO & $\dot{0}$ & & \\
\hline 15-May-97 & Unimportant & $291 \mathrm{BK}$ & INSTRUMENTATION BUILDING & BWHC & B-PLANT & & & 9 & $\mathrm{PCO}$ & 0 & & \\
\hline 15-May-97 & Onimportant & $292 \mathrm{~A}$ & PUREX GASEOUS EFFLUENT MONITORNG BLDG & BWHC & PUREX & & & & FCO & 0 & & \\
\hline 15-May-97 & Unimportant & $292 \mathrm{AA}$ & PR STACK SAMPIE HOUSE & BWFC & PUREX & & & 10 & PCO & 0 & & \\
\hline 15-May-97 & Unimportant & $292 \mathrm{AB}$ & GASES EFFLLNT MONITOR BUILDING & BWHC & PUREX & & & 148 & $\mathrm{PCO}$ & 0 & & \\
\hline 15-Mry-97 & Unimportunt & $293 \mathrm{~A}$ & OFFGAS TREATMENT FACIUTYY & BWHC & PUREX & & & 160 & $\overline{P C O}$ & 0 & & \\
\hline 15-Mry-97 & Unimportant & $294 \mathrm{~A}$ & OFFGAS TREATMENT AND MONNTORING STATION & BWHC & PUREX & & & 6 & PCO & 0 & & \\
\hline 15-Moy-97 & Inh. Rugged & $294 \mathrm{~B}$ & BACKFLOW PREVENTOR BUILDING & BWHC & WESF & 1987 & & 29 & PCI & 0 & GOOD & POOR \\
\hline 15-Mey-97 & Unimportant & $295 \mathrm{~A}$ & ASD SAMPIE STATTON & BWHC & PUREX & & & 6 & PC0 & $\overline{0}$ & & \\
\hline 15-May-97 & Unimportant & $295 \mathrm{AA}$ & SCD SAMPIE AND PUMPOUT STATTON & BWHC & PUREX & & & 8 & PCO & $\overline{0}$ & & \\
\hline 15-Mky-97 & Onimportant & $295 \mathrm{AB}$ & PDD SAMPLE STATION & BWHC & PUREX & & & 14 & PCO & 0 & & \\
\hline 15-May-97 & Unimportant & $295 \mathrm{AC}$ & CSL SAMPLE STATTON & BWHC & PUREX & & & 8 & $\mathrm{PC} 0$ & 0 & & \\
\hline 15-May-97 & Unimportant & 295AD & SWL SAMPLE STATION & BWHC & PUREX & & & 8 & $\overline{P C O}$ & 0 & & \\
\hline $15+$ May-97 & Unimportant & 295AE & PDD MONITORING BLDG & BWHC & PUREX & & & 20 & $\mathrm{PCO}$ & $\mathbf{0}$ & & \\
\hline 15-May-97 & Unimportant & $296 \mathrm{AI}$ & STACK, $202 \mathrm{~A}$ N AND Q CEIIS EXHAUST & BWHC & PUREX & & & & $\mathrm{PCO}$ & 0 & & \\
\hline 15-May-97 & Unimportant & $296 \mathrm{A14}$ & STACK, 293A OFF GASTRTMNT/RECOVERY BLDG & BWHC & PUREX & & & & PCo & $\overline{0}$ & & \\
\hline 15-Moy-97 & Unimportant & $296 \mathrm{A2}$ & STACK, 202A GAIIFRY HOODS EXHAUST & BWHC & PUREX & & & & $\overline{P C O}$ & 0 & & \\
\hline 15-May-97 & Unimportant & $296 \mathrm{~A} 24$ & $\begin{array}{l}\text { STACK. AMMONIA-SCRUBBER WASTE } \\
\text { CONCENIRATOR }\end{array}$ & BWHC & PUREX & & & & $\overline{P C O}$ & 0 & & \\
\hline 15-May-97 & Unimportant & $296 \mathrm{~A}^{3}$ & STACK, 202A SAMPLE GALIERY EXHAUST & BWHC & PUREX & & & & PC0 & 0 & & \\
\hline 15-May-97 & Unimportant & 296ASA & STACK, 202A LABORATORY HOODS EXHAUST & BWHC & PUREX & & & & PCO & $\mathbf{0}$ & & \\
\hline 15-May-97 & Unimportant & $296 \mathrm{ASB}$ & STACK, 202A LAB̈BOTACTORY HOODS EXHAUST & BWHC & PUREX & & & & PCO & 0 & & \\
\hline 15.May-97 & Unimportant & $296 \mathrm{A6}$ & STACK, 202A SAMPLE GALIERYK CELL EXHAUST & BWRC & PUREX & & & & $\mathrm{PCO}$ & 0 & & \\
\hline 15-May-97 & Unimportant & $29 \overline{987}$ & $\begin{array}{l}\text { STACK, PUREX WEST SAMP GALLERY/RCEIL } \\
\text { EXHAUST }\end{array}$ & BWHC & PUREX & & & & $\mathrm{PC} 0$ & $\overline{0}$ & & \\
\hline $15-$ May-97 & Unimportant & $296 \mathrm{A8}$ & $\begin{array}{l}\text { STACK, PUREX PLT PIPE, OPERGALEWT RM } \\
\text { EXHAUST }\end{array}$ & BWHC & PUREX & & & & PCO & 0 & & \\
\hline 15-May-97 & Unimportant & $304 \mathrm{~A}$ & $\begin{array}{l}\text { URANIUMM CONCRETION CHANGE } \\
\text { ROOM/LABORATORY }\end{array}$ & BWHC & FFF & & & 18 & PC0 & 0 & & \\
\hline
\end{tabular}




\begin{tabular}{|c|c|c|c|c|c|c|c|c|c|c|c|c|}
\hline $\begin{array}{l}\text { WHEN } \\
\text { COMPLY }\end{array}$ & COMPLY & IDENTIFIER & DESCERTFION & CONTR & PROJECT & $\begin{array}{l}\text { YEAR } \\
\text { BUILT }\end{array}$ & YEAK & $\begin{array}{l}\text { AREA } \\
\text { SQI } \\
\text { METER } \\
\end{array}$ & $\begin{array}{l}\text { PERE. } \\
\text { CAT. }\end{array}$ & $\begin{array}{l}\text { TŌTAL } \\
\text { OCCUP }\end{array}$ & $\begin{array}{l}\text { FACIITY } \\
\text { CONDInON } \\
\text { RATING } \\
\end{array}$ & $\begin{array}{l}\text { EXISIING } \\
\text { ANALYSIS } \\
\text { RATING } \\
\end{array}$ \\
\hline $15-$ May-97 & Unimportant & $\begin{array}{l}\text { 305WELLSI } \\
\text { AN }\end{array}$ & 305 WEL ISIAN WAY, RICHLAND -OFFICE BUILDING & 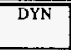 & GPF & & & $\overline{50 \times 0}$ & PCo & 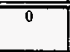 & & \\
\hline $15 \cdot$ May-97 & Unimportant & $\begin{array}{l}\text { 307WEIISI } \\
\text { AN }\end{array}$ & 307 WEI ISIAN WAY, RICHILAND WAREHOUSE & DYN & GPF & & & 5000 & $\overline{P C O}$ & 0 & & \\
\hline 15. May-97 & Init. Rugged & 310 & BUILDING TREATED EFFLUENT DISROSL & RFSH & LEF & 1994 & 2027 & 1255 & PC1 & 9 & LIKE NEW & POOR \\
\hline 15-May-97 & Inh. Kugged & 312 & RIVER PUMP HOUSE & DYN & GPF & 1960 & & 44 & Fi & 0 & GOOD & POOR \\
\hline 15.May-97 & Unimportant & 321 & ENGINEERING DEVELOPMENT LAB & DYN & $\overline{\mathrm{GPF}}$ & 1944 & & 2552 & $\mathrm{PCO}$ & $\overline{0}$ & & \\
\hline 15-May-97 & Unimportarit & $324 \mathrm{~A}$ & CHEMICAL ENGINEARING BUILDING & BWHC & FFF & 1959 & & 24 & PCO & 0 & & \\
\hline 15-May-97 & Inh. Rugged & 3240 & EFFLUENT MONTTORING STATION & BWHC & FFF & 1992 & & 92 & FCI & $\overline{0}$ & IKEE NEW & ACCEPTABIF \\
\hline 15-Mny-97| & Unimportant & 334 & $\begin{array}{l}\text { PROCESS SEWER MONITOR FACIUTYY. CHEXICAL } \\
\text { STORAGE HUILDING }\end{array}$ & BWHC & FFF & 1961 & & 28 & ROO & 0 & & \\
\hline 15-May-97 & Unimportint & $334 \AA$ & $\begin{array}{l}\text { WASTE ACID STORAGE BUILDING, TANKS } \\
\text { (2TANKS), CHEMICAL WASTE RECIEVING } \\
\end{array}$ & BWHC & FFF & 1959 & & 28 & $\overline{P C O}$ & 0 & & \\
\hline 15-Mby-97 & Inh. Kugged & 342 & COLLECTON SUMP 1 - 300 AREA TEDF & RFSH & LEF & 1994 & 2027 & 21 & FCI & 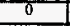 & DKE NEW & POOR \\
\hline 15-May-97 & Inh. Rugged & $342 \mathrm{~A}$ & INSTR/ELEC BUIILDING SHOP & RFSH & LEF & 1994 & 2027 & 16 & PC1 & 0 & IIKE NEW & POOR \\
\hline 15-May-97 & Inh. Ruggad & $3506 \mathrm{~A}$ & POWERHOUSE MAINTENANCE & $\overline{\mathrm{DYN}}$ & GPF & 1951 & 2007 & 174 & FC1 & 3 & GOOD & POOR \\
\hline 15-May-97 & Inh. Rugzad & $3506 \mathrm{~B}$ & MAINTENANCE SHOP & DYN & $\mathrm{GPF}$ & 1973 & 2007 & 80 & FC1 & 4 & GOODD & POOR \\
\hline 15-May-97 & loh Kugged & $3621 \mathrm{~B}$ & EMERGENCY GENERATOR HOUSING & DYN & GPF & 1986 & & 37 & PC1 & 0 & GOOD & POOR \\
\hline 15-May-97 & Inh. Rugged & $3621 \mathrm{C}$ & EMERGENCY GENERATOR HOUSING & $\overline{D Y N}$ & GPF & 1986 & & 24 & $\overline{\mathrm{FC1}}$ & 0 & GOOD & POOR \\
\hline 15-May-97 & Inh. Rugged & 36210 & EMERGENCY GENERATOKOR BUILING & DYN & GPF & 1974 & & 237 & $\overline{\mathrm{PCI}}$ & 0 & GOOD & POOK \\
\hline 15-Mny-97 & Unimportent & $3701 \mathrm{~L}$ & GUARD HOUSE, WISCONSIN ST. & DYN & GPF & & & 126 & $\mathrm{PCO}$ & 0 & & \\
\hline 15-Mny-97 & Unimportant & 3710A & OLLSTORAGE BUIDING & DYN & GPF & 1961 & & 25 & $\mathrm{FCO}$ & 0 & & \\
\hline 15-May-97 & Unimportant & 3715 & STORAGE BUILDINGSTORAGE BUILDING & DYN & GPF & 1959 & & 595 & $\mathrm{PCO}$ & 0 & & \\
\hline 15-May-97 & Unimportant & $37 \mathrm{ITC}$ & MATERIAIS ARCHIVE BUILDING & DYN & GPF & 1970 & & 214 & PCO & $\overline{0}$ & & \\
\hline 15 -May-97 & Unimportant & 3718 & $\mathrm{R}+\mathrm{U}$ SERVICES & DYN & GPF & 1951 & & 355 & $\mathrm{PCO}$ & 0 & & \\
\hline 15-May.97 & Unimportant & $3718 \mathrm{E}$ & STOKARAE BULDING & BWHC & FFF & 1964 & & 1219 & FCO & 0 & & \\
\hline 15-May-97 & Inh. Rugged & $3719 \mathrm{~A}$ & MODULAR FRST AID STATION & DYN & GPF & 1994 & 2027 & 172 & PC1 & 3 & LKE NEW & POOR \\
\hline 15-May-97 & \begin{tabular}{|l|} 
Unimportant \\
\end{tabular} & 3721 & CLASSIFIFD INCINERATOR FACIITTY & DYN & GPF & 1965 & & 22 & $\mathrm{PCO}$ & 0 & & \\
\hline 15-May-97 & Unimportant & $3726 \mathrm{~A}$ & EMERGENCY AIR BOTTLE BLDG (ATTTO 3701D) & DYN & GPF & & & 37 & $\mathrm{PCO}$ & 0 & & \\
\hline 15-May-97 & Unimporiant & 3727 & ARCHIVE FISSILEVAULT & DYN & GPF & 1975 & & 81 & PCO & 0 & & \\
\hline 15-May-97 & Unimportant & 3732 & STORAGE BUIIDING & DYN & GPF & 1949 & & 127 & $\overline{\mathrm{PCO}}$ & 0 & & \\
\hline $15-M_{6 y-97}$ & Unimportant & 3734 & MAINTENANCE STORAGE BUILDING & DYN & GPF & 194 & & 22 & $\mathrm{PCO}$ & 0 & & \\
\hline 15+Mny-97 & Enimportant & $3734 \mathrm{~A}$ & PAINT STORAGE BUILDING & DYN & $\overline{\mathrm{GPF}}$ & 1944 & & 73 & $\overline{\mathrm{PCO}}$ & $\mathbf{0}$ & & \\
\hline 15-May-97 & Oaimportunt & 3765 & OFFCE BULDDING & DYN & GPF & 1955 & & 1131 & PCO & 0 & & \\
\hline 15-May-97 & Inh. Rugged & 382 & PUMP HOUSE BUIDDING & DYN & GPF & 1943 & & 106 & $\mathrm{PCl}$ & $\overline{0}$ & GOOD & POOR \\
\hline 15-May-97 & Inh. Rugged & $382 \mathrm{~B}$ & WATER TANK & $\overline{D Y N}$ & GPF & 1990 & & 136 & $\overline{\mathrm{PCI}}$ & 0 & UKE NEW & POÓR \\
\hline 15-Mry-97 & Inh. Rugged & 3906 & SANITARY \& PROCCESS LJFT BUILDING & DYN & GPF & 1960 & & 26 & PC1 & 0 & GOOD & POŌE \\
\hline 15-May-97 & Inih. Kugged & $3906 A$ & SANITARY SEWER CONTROL BUILDING & DYN & GPF & 1960 & & 174 & RCI & 0 & GOON & POOR \\
\hline 15-May-97 & Inh. Rugged & $3906 \mathrm{~B}$ & SANITARY SEWER LUFT & DYN & GPF & 1960 & & 23 & $\mathrm{PCl}$ & $\mathbf{0}$ & GOOD & POOR \\
\hline 15-May-97 & Inh, Ruggad & 402 & SODIUMM STORAGE BUILDING & 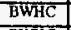 & FFIF & 1996 & & 1310 & PC1 & 0 & UKE NEW & VERIFIABLE \\
\hline $15-\mathrm{May}-97$ & Unimportant & $409 \mathrm{~A}$ & CLOSED LOOP HEAT DUMP, EAST & BWHC & FFTF & & & 74 & $\overline{P C O}$ & $\mathbf{0}$ & & \\
\hline
\end{tabular}




\begin{tabular}{|c|c|c|c|c|c|c|c|c|c|c|c|c|}
\hline $\begin{array}{l}\text { WHEN } \\
\text { COMPLY }\end{array}$ & $\begin{array}{l}\text { HOW } \\
\text { COMPLY }\end{array}$ & IDENTTFER & DESCRIPTION & $\overline{\text { CONTR }}$ & PROJECT & $\begin{array}{l}\text { YEAR } \\
\text { BUILT }\end{array}$ & $\begin{array}{l}\text { YEAR } \\
\text { SURPLUS }\end{array}$ & $\begin{array}{l}\text { AREA } \\
\text { SQ } \\
\text { METER }\end{array}$ & $\begin{array}{l}\text { PERF. } \\
\text { CAT. }\end{array}$ & $\begin{array}{l}\text { TOTAL } \\
\text { OCCUP }\end{array}$ & $\begin{array}{l}\text { FACILTY } \\
\text { CONDITION } \\
\text { RATING }\end{array}$ & $\begin{array}{l}\text { EXISTYING } \\
\text { ANALYYSIS } \\
\text { RATING }\end{array}$ \\
\hline 15-May-97 & Unimportant & $409 \mathrm{~B}$ & CLOSED LOOP HEAT DUMP, EAST & BWHC & $\overline{\text { FFIF }}$ & & & $\overline{74}$ & $\overline{\mathrm{PCC} 0}$ & $\overline{0}$ & & \\
\hline 15.May-97 & Inh. Rugged & 427 & FUELS \& MATERIAL EXAMNATIONFACIUTYFMEF & BWHC & FFF & 1984 & 2037 & T5794 & Fe1 & 4 & GOOD & VERTFIABCE: \\
\hline $15-\mathrm{May}-97$ & Inh. Rugged & $427 \mathrm{~A}$ & $\begin{array}{l}\text { ARGON/HYDROGEN MIXING } \\
\text { BUILDINGARGON/HYDROGEN MIXING BUILDING }\end{array}$ & BWHC & FFF & 1984 & 2037 & 13 & $\overline{P C 1}$ & 0 & UIKE NEW & FOOR \\
\hline \begin{tabular}{|l|}
$15-\mathrm{May}-97$ \\
\end{tabular} & Unimportant & $432 \mathrm{~A}$ & GAS BOTTTLE STORAGE & BWHC & FFTF & & & 93 & PCO & 0 & & \\
\hline 15-May-97 & Unimportant & $4701 \mathrm{~A}$ & FORMER GUARD STATION & BWHC & FFTF & & & 134 & PCO & 0 & & \\
\hline \begin{tabular}{|l|}
$15-$ May-97 \\
\end{tabular} & Unimportant & $4701 B$ & $\begin{array}{l}\text { GUARD STATION, GRANT AVE.UNKKNOWN } \\
\text { BUILDING }\end{array}$ & DYN & GPF & & & 59 & PCO & 0 & & \\
\hline $\mid 15-$ May-97 & Inh. Ruggod & $4701 \mathrm{C}$ & $\begin{array}{l}\text { GUARD STATION, HAYES ST. GAURD STATION - } \\
\text { FFTF }\end{array}$ & BWपC & FFF & 1984 & 2037 & 167 & $\overline{\mathbf{P C 1}}$ & 7 & $\overline{G O O D}$ & MARGINAL \\
\hline 15-May-97 & Unimportant & $4704 \mathrm{~N}$ & SECURTTY MAINTENANCE SHOP OFFCE BUILDING & DYN & GPF & & & 744 & $\mathrm{PCO}$ & 0 & & \\
\hline 15-May-97 & Inh. Ruggod & $4709 \mathrm{~A}$ & FIRE STATION, HOSE STORAGE & DYN & GPF & 1984 & 2007 & 837 & PC1 & 5 & GOOD & FOOK \\
\hline 15-May-97| & Unimportant & 4719 & MEDICAAL AID STATION & DYN & GPF & & & 171 & PCO & $\overline{0}$ & & \\
\hline 15-May-97 & Unimportant & 4726 & JANITOWALA STORAGE & BWHC & FFIF & & & 30 & FCo & 0 & & \\
\hline 15-May-97 & Unimportant & 47328 & WAREHOUSE & BWHC & FFTF & & & 2230 & $\mathbf{P C 0}$ & $\overline{0}$ & & \\
\hline 15-May-97 & Unimportant & $4732 \mathrm{C}$ & WAREHOUUSE & BWHC & FFTF & & & 1859 & FCO & \% & & \\
\hline 15-May-97 & Unimportant & $47 \overline{3} 4 \bar{C}$ & WAREHOUSE (SPECIAL TOOLS) STORAGE BUILDING & DYN & GPF & & & 735 & Fo & 0 & & \\
\hline 15-May-97 & Uaimportant & $4734 \mathrm{D}$ & WAREHOUSE & DYN & GPF & & & 735 & PCO & 0 & & \\
\hline \begin{tabular}{|l|}
$15-M a y \cdot 97$ \\
\end{tabular} & Unimportant & $480 \mathrm{~A}$ & WATER WELL NO. 1 & BWHC & FFTF & & & 15 & PCo & 0 & & \\
\hline 15-May-97 & Unimportant & $480 \mathrm{~B}$ & WATER SUPPLY WELL HOUSE & BWHC & FFTF & & & 15 & PCO & 0 & & \\
\hline 15-May-97 & Unimportaint & 4800 & WATER SUPPY WELL HOUSE NO. 3 & BWHC & FFTF & & & 25 & FCO & 0 & & \\
\hline \begin{tabular}{|l|} 
I5-May-97 \\
\end{tabular} & Inh. Rugged & $483 \mathrm{~B}^{-1}$ & $\begin{array}{l}\text { WATER TREATMIENT BUILDINGWATERR } \\
\text { TREATMENT BUILDING }\end{array}$ & BWHC & FFF & 1984 & & 42 & FCI & 0 & GOOD & ACCEPTABDE \\
\hline 15-May-97 & Unimportant & $4842 \mathrm{~B}$ & SWITCH WATER PUMPHOUSEWATER PUMP HOUSE & BWHC & FFTF & & & 28 & $\overline{\mathrm{FCO}}$ & $\dot{0}$ & & \\
\hline 15-May-97 & Unimportant & 4843 & AIKALAI METAL STORAGE & BWHC & FFTF & & & 150 & PCO & 0 & & \\
\hline 15-May-97 & Inh. Rugged & $604 \mathrm{~A}$ & YAKIMA BARRICADE, PATROL SENTRY & DYN & GPF & 1985 & 2017 & 22 & FI & $T$ & GOOD & POOR \\
\hline 15-May-97 & Unimportant & 604G & WYE BARRICADE, PATROL, UTTUTYY BUILDING & DYN & GPF & & & 9 & $\mathrm{PCO}$ & 0 & & \\
\hline \begin{tabular}{|l|}
$15-M a y-97$ \\
\end{tabular} & Unimportinnt & 6265 & $\begin{array}{l}\text { ENVIRO NMENTAL SUPPORT T IABORATORY UTILTY } \\
\text { BLDG }\end{array}$ & RFSH & HAS & 1993 & 2024 & 335 & PCO & 0 & IIKE NEW & MAKGINAL \\
\hline 15-May-97 & Unimportant & $6265 \mathrm{~A}$ & SOLD WASTE STORAGE BUIDDING & RFSH & HAS & 1993 & 2024 & 67 & PCO & 0 & DKKENEW & POOK \\
\hline 15-Mny-97 & Unimporthnt & $6266 \mathrm{~A}$ & $\begin{array}{l}\text { CONTAMINATED UQULD WASTE RETENTION } \\
\text { VAULT }\end{array}$ & RFSH & HAS & 1993 & 2024 & 67 & $\overline{\mathrm{PCO}}$ & 0 & LIKE NEW & VERIFIED \\
\hline 15-May-97 & Inh. Ruszod & 6267 & ENVIRONMENTAL SAMPLE ARCHIVE BUILDING & RFSH & HAS & 1993 & 2024 & 167 & PCI & 0 & LIKE NEW & MARGINAL \\
\hline 15-May-97 & Inh. Kuzzed & 6268 & SAMPLE EQUIPMEN'T CIEANING (Euture) & RFSH & HAS & 1995 & 2026 & 223 & FCI & 4 & LKEENEW & ACCEPTABLE \\
\hline 15-Mny-97 & Inh. Rugged & 6269 & MOBIIE LABORATORY STORAGE & RESH & HAS & 1993 & 2024 & 632 & PCl & 9 & LXKE NEW & MARGINAL \\
\hline 15-May-97 & Inh. Rugged & 6653 & SAMPLE AND MONITORING BUIIDING & RFSH & LEF & 1994 & 2025 & 47 & PC1 & 5 & LIKE NEW & POOR \\
\hline 15-Mny-97 & Inh. Rugzed & 6701 & WYE BARRICADE GUARD HOUSE & DYN & GPF & 1992 & 2027 & 24 & $\overline{\mathrm{PC} I}$ & 1 & UKE NEW & FOR \\
\hline 15-Mny-97 & Thh. Kugged & 67018: & SR240/2WIBARRCADE & DYN & GPF & 7994 & 2017 & 2 & PCI & 1 & IIKE NEW & POOR \\
\hline 15-Mny-97 & Unimportent & MOO06 & MOBIL L OFFCE (377 TRL 1) & DYN & GPF & 1974 & & 62 & PCO & 0 & & \\
\hline 15-Mny-97 & Unimportant & MO009 & MOBILE OFFCE (3745 TRL 1) & DYN & GPF & 1977 & & 62 & $\mathrm{PCO}$ & 0 & & \\
\hline 15-May-97 & Unimportant & MOŌI & TANTTOR STGE/OFC @ 284E & DYN & GPF & 1976 & 2007 & 62 & PCO & 0 & MARGINAL & POOR \\
\hline
\end{tabular}




\begin{tabular}{|c|c|c|c|c|c|c|c|c|c|c|c|c|}
\hline & & 0 & 0.2 .1 & & & & dVyM & HSTY & 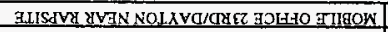 & S900N & \begin{tabular}{|l|l|} 
Ingrodumg $\mathrm{n}$ \\
\end{tabular} & L6, *KEWN-SI \\
\hline & & 0 & $0.0 \mathrm{~d}$ & 79 & & & dram & HSTO & FOHHO TIISOWYTIIVAL & tgrow & 1truodanu & L6-KWW-SI \\
\hline & & 0 & $0.0 \mathrm{dI}$ & 29 & & & dVaM & HSEY & 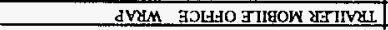 & Strow & ztrupodanu & $26-6 \times W \cdot W \cdot 51$ \\
\hline & & e & $0,2 \times$ & $\varepsilon s$ & & & swam & HSTH & 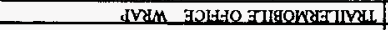 & thon & guruoduryn & L6-KEW-S1 \\
\hline $800 \mathrm{~d}$ & MaN BXXI & $\varepsilon$ & 129 & Is & $\angle 00 z$ & $\varepsilon 66 \mathrm{I}$ & तथा & HSHA & 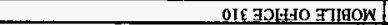 & EthoN & posing 'yuI & $L 6-k=N-S I$ \\
\hline good & g000 & $\varepsilon$ & LOd & SIS & 2.02 & $\$ 861$ & Fd 9 & NXG & 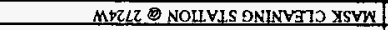 & ZTHON & pos8ny - पण & L6-kwW-SI \\
\hline & & 0 & $0,0 \mathrm{~d}$ & 951 & & 2861 & $\operatorname{sd} 0$ & NSO & (S THL LIWU) GOHAO TTIRON & $5650 W$ & guevoduning & L6-KWW-SI \\
\hline & & 0 & $02 \mathrm{~d}$ & zII & & $286 \mathrm{~L}$ & $\mathrm{JdQ}$ & NSOC & 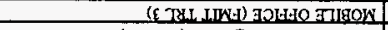 & profon & $\begin{array}{ll}\text { inspodurana } \\
\end{array}$ & L6-k0y-SI \\
\hline & & 0 & $0,02 \mathrm{~d}$ & $z_{\ell \mathrm{I}}$ & & & Id 0 & NKa & 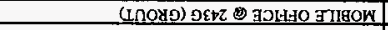 & $26 \varepsilon O N$ & Trentodung & L6-KaW-SI \\
\hline & & 0 & $0.0 \mathrm{~d}$ & $z L I$ & & & JdD 0 & NXO & 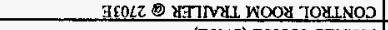 & L8EOW & 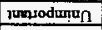 & L6-KEW'SI \\
\hline & & 0 & $0.2 \mathrm{~d}$ & $z L I$ & & & 메을 & NXI & 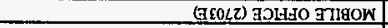 & t8EON & proprodurion & L6-KEWT-SI \\
\hline & & 0 & 028 & 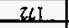 & & & tdid & N60 & 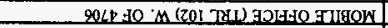 & $66 \mathrm{gON}$ & 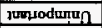 & $66^{-K 5 y} W-5 I$ \\
\hline & & 0 & 020 & $z<\mathrm{I}$ & & & IdD $D$ & NXa & 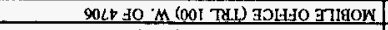 & BLEOW & provodumun & $\angle 6-K=\mathrm{W}-\mathrm{SI}$ \\
\hline & & 0 & $0.2 \mathrm{~d}$ & $\iota^{+}$ & & & Ad 0 & nXG & (LILZ) WOO४ ЗอNWHO JTIRONA & ILEON & jugprodmen & L6-KEW-5I \\
\hline & & 0 & $0.0 \mathrm{~d}$ & ZII & & & sdo & $\mathrm{NAO}$ & SOLT IO 'M 3DHAO ETIRON & ¿\$६ON & gronioduring & $\angle 6-\operatorname{SuHN}-\mathrm{SI}_{1}$ \\
\hline & & 0 & $02 d$ & 82 & & & Add & $\mathrm{N} \Omega \mathrm{G}$ & 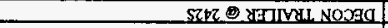 & 93600 & zurpodunun & L6-SesW-SI \\
\hline & & 0 & $0,0 \mathrm{~d}$ & $\frac{8 \tau}{2}$ & & & sdD & nAd & 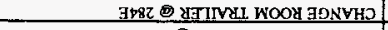 & Dz\&ON & Trmodung 0 & $\angle 6^{-K k} \mathrm{~W} \cdot \mathrm{SI}$ \\
\hline & & $\frac{0}{0}$ & 0.021 & $\frac{82}{25}$ & & & LNYId-g & $2 \mathrm{Hing}$ & HLOZ Q RTIVII JONVHO dMS & zzkoN & 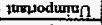 & $\angle 6-\mathrm{SEW}-\mathrm{SI}$ \\
\hline & & 0 & 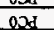 & $\frac{58}{78}$ & & & $\frac{\mathrm{AdO}}{\mathrm{sdO}}$ & $\frac{N R Q}{N S O}$ & 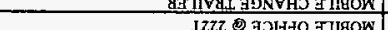 & sison & 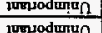 & 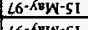 \\
\hline TYNISYVW & MAN TXYI & 6 & IDd & $9 \varepsilon$ & & to65 & WMS & HSSA & 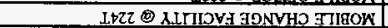 & $68 \mathrm{zON}$ & posingy - परा & 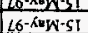 \\
\hline TVNIOYYK & MIN उत्रता & 6 & זוגלו & 98 & & 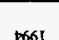 & wins & HSSH & $\begin{array}{r}\text { XFIdWO2 } \\
\end{array}$ & | & pef8nx & $x=0$ \\
\hline & & 0 & 020 & OLL & & 2665 & STAI & JHMg & 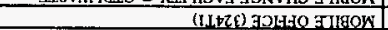 & DLZON & ztrompodunun & 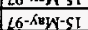 \\
\hline $40 O_{A I}$ & MaN สXXII & $s$ & iDd & $z L I$ & & 1661 & सहा & HSFम & 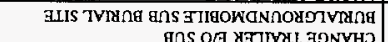 & $6970 \mathrm{~W}$ & po88ny - vu & \\
\hline & & 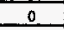 & $0.2 \mathrm{~d}$ & $Z L I$ & & 0661 & AdD & NAg & 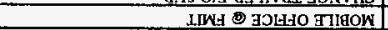 & [9zow & zusyodunun & 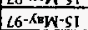 \\
\hline & & $\theta$ & $02 d$ & 241 & & 0661 & IdP & באה & 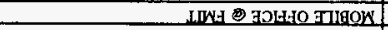 & 0920W & 2urpodumun & $66-x+x-5]$ \\
\hline $\mathrm{yOOAd}$ & 0000 & $t$ & $10 d$ & $z L I$ & 1002 & 0661 & IdD & NXC & 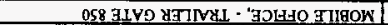 & EszOW & 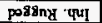 & L6-KEW-SI \\
\hline $800 \mathrm{~d}$ & 0000 & 0 & I0d & $z 41$ & $\angle 000$ & 00651 & Id 9 & NXI & 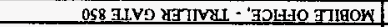 & zSzON & posing पष्य & C6-KUWLSI \\
\hline $800 \mathrm{~d}$ & 0000 & 8 & 10d & $Z L I$ & $L 002$ & 0661 & $\mathrm{dd} D$ & $\mathrm{NSO}$ & 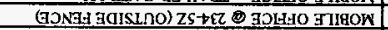 & $6+20 \mathrm{~N}$ & pos?ng 'quI & 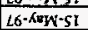 \\
\hline 8000 & 9002 & 6 & i2d & $Z \angle L$ & 2002 & 0661 & 392 & $N \alpha Q$ & 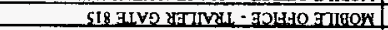 & $8+200$ & 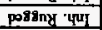 & 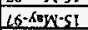 \\
\hline HoOd & 0000 & 6 & IDd & $z_{L 1}$ & $\angle 00 z$ & $066 \mathrm{I}$ & tad & $\mathrm{NAO}$ & 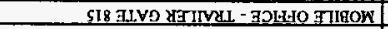 & $9+20 N$ & posing 'чU & $16-6 \ln w$ \\
\hline $\mathrm{NOOA}$ & 0000 & 8 & IXI & थ! & 2002 & 0661 & dde & $\mathrm{NhO}$ & 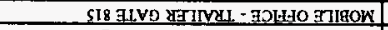 & stzon & po82ny ' पण & $\angle 6-K \nabla W-S I$ \\
\hline $800 \mathrm{~d}$ & 9000 & 0 & I:2I & 852 & $\angle 102$ & $106 \mathrm{I}$ & HdD & NAG & $2 S$ t $\varepsilon Z$ Q & Dt2ON & polsing पुपा & $\angle 6-\hat{x} \mathrm{EW}-\mathrm{SI}$ \\
\hline 8000 & 9000 & I & 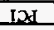 & ILI & 4102 & 9861 & $\$ d Q$ & sug & 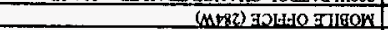 & $0+20 w$ & posing quy & 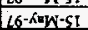 \\
\hline Yyo0d & a009 & I & 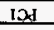 & ILI & $L 102$ & $986 \mathrm{I}$ & AdD & $\mathrm{NAC}$ & 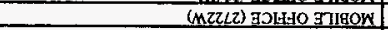 & SEzow & pos?ng *4य] & 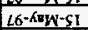 \\
\hline & & e & $0.2 \mathrm{~d}$ & 29 & & & ANDO & $\mathrm{NAC}$ & 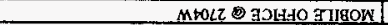 & IzZOW & Yrewodung 0 & $\angle \sigma^{-}-\hat{\omega n y}$ \\
\hline & & 0 & $00 \mathrm{~d}$ & 29 & $z 00 z$ & & ddD & NSG & 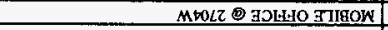 & sIzOW & Jumiodumen & $\angle 6-S B W \cdot 5 T$ \\
\hline & & 0 & 020 & $\angle+1$ & & & HAD & $\mathrm{NSG}$ & 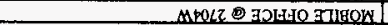 & $9500 \mathrm{~N}$ & 7usphodumun & $6 \sigma^{-60} \mathrm{~W}_{\mathrm{K}}-\mathrm{S}_{1}$ \\
\hline & & 0 & 0.01 & $z L I$ & & 1861 & IdO & NSO & 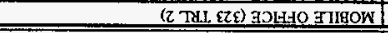 & ISOOW] & juovodunion & $\angle 6-\overline{S H W} \mathrm{SI}$ \\
\hline $\begin{array}{l}\text { ONIIVY } \\
\text { SISXTVNV } \\
\text { ONILSIXI }\end{array}$ & $\begin{array}{r}\text { DNLUY } \\
\text { NOLIGNOJ } \\
\text { NIIIVYS }\end{array}$ & $\begin{array}{l}\text { dnDDo } \\
\text { TYLOL }\end{array}$ & $\begin{array}{r}\text { IVD } \\
\text { ISTRA }\end{array}$ & 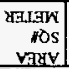 & $\begin{array}{r}\text { snzdras } \\
\text { yved } \\
\end{array}$ & $\begin{array}{l}\text { L7Lר } \\
\text { YYis }\end{array}$ & aroudd & X.LNOO) & NoLdarosad & MALLLNAGI & $\begin{aligned} \text { NTAWOS } \\
\text { MOH }\end{aligned}$ & $\begin{aligned} X \supset \text { JWOO } \\
\text { NFHM }\end{aligned}$ \\
\hline
\end{tabular}




\begin{tabular}{|c|c|c|c|c|c|c|c|c|c|c|c|c|}
\hline $\begin{array}{l}\text { WFEN } \\
\text { COMPLY }\end{array}$ & $\begin{array}{l}\text { HOW } \\
\text { COMPLY }\end{array}$ & TIDENTIFER & DESCRIFTON & CONTR & TRKOJECT & $\begin{array}{l}\text { YEAR } \\
\text { BUILT }\end{array}$ & $\begin{array}{l}\text { YEAAR } \\
\text { SURPLUS }\end{array}$ & $\begin{array}{l}\text { AREA } \\
\text { SQA } \\
\text { METER }\end{array}$ & $\begin{array}{l}\text { PERF. } \\
\text { CAT. }\end{array}$ & TOTAL & $\begin{array}{l}\text { FACIIITY } \\
\text { CONDITION } \\
\text { RATTNG }\end{array}$ & $\begin{array}{l}\text { EXISTING } \\
\text { ANALYSIS } \\
\text { RATTNG }\end{array}$ \\
\hline 15-Miny-97 & Unimportant & MOS37 & CHANGE TRAILER (O) 22LA & BWHC & B-PLANT & & & $\overline{40}$ & $\overline{100}$ & 8 & & \\
\hline 15-May-97 & Unimportant & |MOS31 & MOBILE OFC @ BALTIMORE N/O 4TH & DYN & GPF & & & 156 & PCO & 0 & & \\
\hline 15-May-97 & \begin{tabular}{|l|} 
Únimpontant \\
\end{tabular} & MO553 & MOBILE OFFICE ON BALTIMORE N/OATH & DYN & GPF & & & 156 & $\mathrm{PCO}$ & 0 & & \\
\hline 15-May-97 & \begin{tabular}{|l|} 
Unimportant \\
\end{tabular} & MO55s & ESCORT TRAILER @ GATE 814 & DYN & GPF & & & 67 & PCO & $\overline{0}$ & & \\
\hline 15-May-97 & \begin{tabular}{|l|} 
Unimportant \\
\end{tabular} & MO559 & ESCORT TRALLR MOBIIIE OFFCE (ESCORT) & DYN & GPF & & & 52 & $\mathrm{PCO}$ & 0 & & \\
\hline 15-May-97 & \begin{tabular}{|l|} 
Tnimportant \\
\end{tabular} & MO662 & FELD MOBILE @ SLAB YARD & DYN & GPF & & & 48 & PCO & 0 & & \\
\hline 15-May-97 & Unimportant & MO705 & JANITORIAL STORAGE @ $284 \mathrm{E}$ & DYN & GPF & & & 48 & $\mathrm{PCO}$ & 0 & & \\
\hline 15-May-97? & \begin{tabular}{|l|} 
Unimportant \\
\end{tabular} & MO709 & MOBILE OFFCE ALSO KNOWN AS 377TRL & DYN & GPF & 1966 & & 39 & PCO & 0 & & \\
\hline 15-May-97 & Unimportant & MO712 & PESTICIDE STORAGE - $201 \mathrm{~W}$ & DYN & $\overline{\mathrm{GPF}}$ & & & 39 & PCO & 0 & & \\
\hline I5-May-97 & \begin{tabular}{|l|} 
Unimportant \\
\end{tabular} & MO713 & GENWATER TANK @ LANDFILL & DYN & GPF & & & 48 & PCO & 0 & & \\
\hline 15-May-97 & \begin{tabular}{|l|} 
Inh. Rugged \\
\end{tabular} & MO722 & MOBILE OFFCE $\triangle 224 \mathrm{U}$ & DYN & GPF & 1992 & 2007 & 172 & PC1 & 0 & UKE NEW & POOR \\
\hline 15-May-97 & Unimportant & MO739 & $\begin{array}{l}\text { SWP CHANGE TRAILR @ 2706TCHANGE TRAILER - } \\
2706 \mathrm{~T}\end{array}$ & RFSH & T.PLANT & & & 48 & $\mathrm{PCO}$ & 0 & & \\
\hline 15-May-97] & Inh. Rugzed & MO741 & HPT OFFCE - 340HPTOFFCE & RESH & LEF & 1993 & 109 & 22 & PCI & 4 & ZKEENEW & POOR \\
\hline 15-May-97 & \begin{tabular}{|l|} 
Onimportant \\
\end{tabular} & M0747 & MOBILE OFFICE $@ 200$ AREA ETF & DYN & GPF & & & 1152 & $\mathrm{PCO}$ & $\mathbf{0}$ & & \\
\hline 15-Mny-97 & \begin{tabular}{|l|} 
Unimportant \\
\end{tabular} & M0829 & MOBILE OFFCE @ BALTIMORE N/O 4TH & DYN & GPF & & & 156 & $\mathrm{PCO}$ & 0 & & \\
\hline 15-May-97 & \begin{tabular}{|l|} 
Unimportant \\
\end{tabular} & MO835 & MOBILE OFFICE & RFSH & T.PLANT & & & 67 & $\mathrm{PCO}$ & 0 & & \\
\hline 15-May-97 & \begin{tabular}{|l|} 
Tnimportant \\
\end{tabular} & MO845 & LUNCHROOM TRAILER @ SLAB YARD & DYN & GPF & & & 67 & PCO & 0 & & \\
\hline 15-May-97 & \begin{tabular}{|l|} 
Unimportant \\
\end{tabular} & MO901 & MOBIIE OFFCE @ 4TH \& BALTIMORERAKA:2911E) & DYN & GPF & & & 125 & $\mathrm{PCO}$ & 0 & & \\
\hline 15-May-97 & Unimportant & MO902 & MOBILE OFFICE SHOP (306 TRL 7 ) & DYN & GPF & 1976 & & 125 & $\mathrm{PCO}$ & $\overline{0}$ & & \\
\hline 15-May-97 & \begin{tabular}{|l|} 
Unimporlant \\
\end{tabular} & MOQ⿻013 & MOBILE OFHCE (KMIT TRL 9) & DYN & GPF & 1976 & & 125 & $\mathrm{PCO}$ & 0 & & \\
\hline 15-May-97 & \begin{tabular}{|l|l|} 
Unimportanst \\
\end{tabular} & fMO & MOBILE OFFICE W. OF $47 \% 6$ & DYN & GPF & & & 125 & $\mathrm{PCO}$ & 0 & & \\
\hline 15-May-97 & \begin{tabular}{|l|} 
Unimportent \\
\end{tabular} & MO909 & MOBILE OFFCE @ 222T MOBILE OFFCE (222T) & RFSH & T-PLANT & & & 125 & PCO & 0 & & \\
\hline 15-May-97 & \begin{tabular}{|l|} 
Unimportant \\
\end{tabular} & M0921 & MOBBIIE OFFCE @ 4TH \& BALTIMORE(29I1E) & DYN & GPF & & & 125 & $\mathrm{PCO}$ & 0 & & \\
\hline 15-May-97 & \begin{tabular}{|l|l} 
Unimportant \\
\end{tabular} & M0926 & MOBIIE OFFCE (FMTT TRL 4) & DYN & GPF & 1977 & & 125 & $\mathrm{PCO}$ & 0 & & \\
\hline T5-May-97] & Onimportant & MO932 & MOBILE OFHCE (2911E) & DYN & GPF & & & 143 & PCO & 0 & & \\
\hline 15-May-97 & \begin{tabular}{|l|} 
Unimportant \\
\end{tabular} & MO933 & MÖB̈ILE OFHCE (FMIT TRL. 10) & DYN & GPF & 1975 & & 134 & $\mathrm{PCO}$ & 0 & & \\
\hline 15-May-97 & \begin{tabular}{|l|} 
Inh. Rugged \\
\end{tabular} & MO934 & MOBILE OFFICE (284E) & DYN & GPF & 1986 & 1999 & $6 \overline{2}$ & PCi & 2 & GOOD & POOR \\
\hline 15-May-97 & \begin{tabular}{|l|} 
Unimportant \\
\end{tabular} & MOO935 & MOBILE OFFCE (3763 TRL 1) & DYN & GPF & 1977 & & 107 & $\overline{\mathrm{PCO}}$ & 0 & & \\
\hline 15-May-97 & \begin{tabular}{|l|} 
Unimportant \\
\end{tabular} & MO967 & MOAILE OFFCE @ 224B & DYN & GPF & & & 67 & $\mathrm{PCO}$ & 0 & & \\
\hline 15-May-97 & \begin{tabular}{|l|} 
Unimportant \\
\end{tabular} & M0978 & MOBILE OFFICE & DYN & GPF & & & 134 & $\mathrm{PCO}$ & 0 & & \\
\hline 15-May-97 & \begin{tabular}{|l|} 
Unimportant \\
\end{tabular} & MO990 & MOBILE OFFCE (2SIW) & DYN & GPF & & & 65 & $\mathrm{PCO}$ & $\mathbf{0}$ & & \\
\hline 15-May-97) & \begin{tabular}{|l|} 
Unimportant \\
\end{tabular} & TKS50 & COOLING WATER TANK AND PIT & BWHC & WESF & & & & PCO & 0 & & \\
\hline
\end{tabular}


HNF-SD-GN-ER-507, REV 0

This page intentionally left blank.

A-12 
HNF-SD-GN-ER-507, REV 0

\section{APPENDIX B}

COMPLIANCE BASELINE FOR EXISTING BUILDINGS AND STRUCTURES WHERE AN EXEMPTION OR DEVIATION IS BEING REQUESTED 
HNF-SD-GN-ER-507, REV 0

This page intentionally left blank.

B-2 
Appendix B. Compliance Baseline For Existing Buildings and Structures Where An Exemption or Deviation is Being Requested. Total Assets $=310$

\begin{tabular}{|c|c|c|c|c|c|c|c|c|c|c|c|c|}
\hline $\begin{array}{l}\text { WHEN } \\
\text { COMPLY }\end{array}$ & $\begin{array}{l}\text { Exempt } \\
\text { Status }\end{array}$ & TIDENTIFIER & DESCAPTION & CONIRA & Project & $\begin{array}{l}\text { YEAR } \\
\text { BUILT }\end{array}$ & $\begin{array}{l}\text { YEAR } \\
\text { SURPLUS }\end{array}$ & $\begin{array}{l}\text { AREA } \\
\text { SQH } \\
\text { METERS }\end{array}$ & $\begin{array}{l}\text { Perf. } \\
\text { Cat. }\end{array}$ & $\begin{array}{l}\text { TOTAL } \\
\text { OCCUP }\end{array}$ & $\begin{array}{l}\text { FACILIYY } \\
\text { CONDITION } \\
\text { RATHNG }\end{array}$ & $\begin{array}{l}\text { EXISIING } \\
\text { ANALYSIS } \\
\text { RATING }\end{array}$ \\
\hline 15-Mny-97 & EI & 1169 & CHEMICAL STORAGE FACIUTY & $\overline{\overline{\text { DYN }}}$ & $\overline{\overline{G P F}}$ & 1953 & & 214 & $\overline{\mathrm{PCl}}$ & 0 & POOR & $\overline{\text { POOR }}$ \\
\hline 15-May-97 & EI & TFOKRER & WATER STUDIES RECIRCULATKON BUIDDING & DESH & SNF & 1955 & & 232 & $\mathrm{PC1}$ & 0 & MARGINAL & POOK \\
\hline 15-May-97 & El & $181 \mathrm{~B}$ & RIVER PUMP HOUSE - RAW WATER & DYN & GPF & 1960 & & 795 & PCl & 0 & POOR & POOR \\
\hline $\mid 15-$ May-97 & EI & $181 \bar{D}$ & RTVER PUMP HOUSE & DYN & GPF & 1960 & & 795 & PCI & 0 & POOR & POOR \\
\hline 15-May-97 & El & $1901 Y$ & VALVE HOUSE & DYN & GPF & .1960 & & 35 & PCl & 0 & MARGINAL & FOOR \\
\hline 15-Mny-97 & El & 19012 & VALVE HOUSE & DYN & GPF & 1960 & & 35 & PCT & 0 & MARGINAL & POOR \\
\hline $\mid$ 15-May-97| & EI & $202 \mathrm{~A}$ & PUREX CANYON AND SERVICE FACILITY & BWKC & PUREXX & 1956 & & 12080 & PCI & 0 & TOOKR & POOR \\
\hline 15-May-97 & EI & $207 \mathrm{~A}$ & SOUTH RETENTION BASIN & RFSH & $242 \mathrm{~A}$ & 1977 & & & $\mathrm{PCI}$ & 0 & MARGINAL & VERTIED \\
\hline 15-May-97 & $\overline{\mathrm{E}} \mathbf{I}$ & $207 \mathrm{~B}$ & RETENTIION BASIN & BWHC & B-PLANT & 1966 & & 3806 & PCI & 0 & POOR & POOKR \\
\hline 15-May-97 & ET & $207 \mathrm{BA}$ & MONITORING BUILDING & BWHC & B-PLANT & 1986 & & 6 & PCI & 0 & MARGINAL & PODR \\
\hline 15-May-97 & El & $207 \mathrm{SL}$ & WATZR RETENTION BASIN & RFSH & $222-5$ & 1952 & 2027 & 260 & PCI & 0 & POOR & POOR \\
\hline \begin{tabular}{||c|}
$15-$ Mny-97 \\
\end{tabular} & EI & $212 \mathrm{~B}$ & HISSION PRODUCTS LOAD OUT STATION & BWHC & B-PLANT & 1968 & & 408 & PCI & 0 & POOR & ACCËPABLE \\
\hline 15-Miny-97 & $\overline{\text { EII }}$ & 216164 & RETENTKON BASIN & BWHC & B-PLANT & 1974 & & & PC1 & 0 & MARGINAL & POOR \\
\hline 15-May-97 & EI & 218E18 & INACTIVEPUREX TUNNELI & BWHC & PUREX & 1956 & & 800 & PCI & 0 & POOR & POOR \\
\hline 15-May-97 & EI & 218E19 & PUREX TUNNEL 2 & BWHC & PUREX & 1964 & & 3200 & PCI & 0 & MARGINAL & POOR \\
\hline 15-May-97 & El & $221 \bar{B} A$ & 15 INCH COOOLING WATER MONITOR BLDG. & BWHC & B-PLANT & 1966 & & 6 & PC1 & 0 & POOR & POOR \\
\hline 15-Mny-97 & EI & $2218 B$ & PROCESS STEAM AND CONDENSATE BUILDING & BWHC & B-PLANT & 1968 & & 28 & $\overline{\mathrm{PCI}}$ & O & POOR & ROOR \\
\hline 15-May-97 & EI & 221BC & SWP CHANGEHOUSE & BWHC & B-PLANT & 1973 & & 8 & PCI & 0 & MARGINAL & POOK \\
\hline 15-May-97 & EI & $2218 D$ & LAUNDRY STORAGE BUUILDING & BWHC & B-PLANT & 1973 & & 40 & $\overline{\mathrm{PCI}}$ & 0 & MARGINAL & POOR \\
\hline 15-May-97 & El & $2225 B$ & FILTER BUILDNG, HNAC FILTER HOUSE & $\overline{\mathrm{RFSH}}$ & $222-5$ & 1975 & 2027 & 72 & PCI & 0 & MARGINAL & POOR \\
\hline 15-Mry-97 & EI & $222 \mathrm{SC}$ & FILTER BUILDING, NORTH & RFSH & $222-5$ & 1980 & 2027 & 85 & $\overline{\mathrm{PCI}}$ & 0 & MARGINAL & POOK \\
\hline 15-May-97 & El & $2225 \mathrm{D}$ & SOLD WASTE HANDLING AND STORAGE FACIUTY & RFSH & $222-5$ & 1983 & 2027 & 30 & PCI & 0 & MARGINAL & FOOR \\
\hline 15-May-97 & Ei & $222 \mathrm{SF}$ & EQUPMENT STORAGE, SERVICE BUILDING & RFSH & $222-5$ & 1982 & 2027 & 70 & PC1 & 0 & MARGINAL & POOR \\
\hline 15-May-97 & EI & $225 \mathrm{BA}$ & KI FLTER PIT & BWHC & WESF & 1973 & & 59 & FCI & $\overline{0}$ & MARGINAL & ACCEPTABLE \\
\hline 15-May-97 & E1 & $225 \mathrm{BC}$ & COMPRESSOR FACILTY & BWHC & WESF & 1973 & & 94 & FC1 & 0 & MARGINAL & POOR \\
\hline |15-Mry-97 & ET & $225 \mathrm{BD}$ & ENCAPSULAIION WASTE MONITORING AND SAMPLE BUIDDING & BWHC & WESF & 1973 & & 22 & PCI & 0 & MARGINAL & POOR \\
\hline |15-May-97 & E1 & 2312 & MATERIALS ENGINEERING LABORATORY & BWEC & PFP & 1940 & & 4870 & PCI & 0 & POOR & POOR \\
\hline \begin{tabular}{||c|}
$15-$ May-97 \\
\end{tabular} & EI & $242 A 702$ & TURBINE BUILDING & RFSH & $242 \mathrm{~A}$ & 1977 & & 980 & PCI & 0 & MARGINAL & POOR \\
\hline 15-May-97 & EI & $2706 \mathrm{~T}$ & EQUUPMENT DECONTAMINATIONBUILDING & RFSH & T-PLANT & 1959 & & 370 & PC1 & 0 & POOR & ACCEPTABLE \\
\hline 15-May-97 & EI & 27TSE & PAINT STORAGE BUILDING & BWHC & B-PANI & 1965 & & 30 & PCT & 0 & POOR & POOK \\
\hline 15-May-97 & El & 271SED & PAINT STORAGE FACILITY & DYN & GPF & 1980 & & 54 & $\mathrm{PCl}$ & 0 & MARGINAL & POOR \\
\hline 15-May-977 & EI & 272BA & DRYY MATERIAL STTORAGE B̈UIDING & BWHC & B-PLANT & 197 & & 89 & PCI & 0 & POOR & POOR \\
\hline 15-May-97 & EI & $272 \overline{B B}$ & INSULATION STORAGE BUILDING & $\overline{\mathrm{BWHC}}$ & B-PLANT & 1971 & & 79 & $\overline{\mathrm{PCI}}$ & 0 & FOOK & POOR \\
\hline 15-May-97 & EI & $27342 \mathrm{~A}$ & GAS SIOKAGE & BWHC & PFF & $?$ & & 10 & PCI & 0 & POOR & POOR \\
\hline 15-May-97 & El & $2734 \mathrm{ZB}$ & GAS STORAGE & BWHC & PFP & $?$ & & 10 & PCI & 0 & POOOR & POOR \\
\hline 15-Mny-97 & E1 & $27392 \mathrm{C}$ & GAS STORAGE BUILDING & BWHC & PFP & 7 & & 10 & PCI & 0 & POOR & POOR \\
\hline 15-Mny-97 & E1 & $2734 Z \mathrm{Z}$ & PROCCESS GAS STŌRAGE & BWHC & PFP & 7 & & 10 & PCl & 0 & POOR & POOR \\
\hline
\end{tabular}




\begin{tabular}{|c|c|c|c|c|c|c|c|c|c|c|c|c|}
\hline $\begin{array}{l}\text { WHEN } \\
\text { COMPLY }\end{array}$ & $\begin{array}{l}\text { Excmpt } \\
\text { Status }\end{array}$ & TDENTMTEK & DESCRPTION & CONTKA & Froject & $\begin{array}{l}\text { YEAR } \\
\text { BUILT }\end{array}$ & $\begin{array}{l}\text { YEAR } \\
\text { SURPLUSS }\end{array}$ & \begin{tabular}{|l|} 
AREA \\
SQ \\
METERS \\
\end{tabular} & Pert. & $\begin{array}{l}\text { TÓTAL } \\
\text { OCCUP }\end{array}$ & $\begin{array}{l}\text { FACILITY } \\
\text { CONDITON } \\
\text { RATING }\end{array}$ & $\begin{array}{l}\text { EUSTING } \\
\text { ANALYSIS } \\
\text { RATING }\end{array}$ \\
\hline 15-May-97 & E1 & $27342 F$ & GAS STORAGE & DWHC & PFP & 7 & & 10 & PCI & 0 & POOR & POOAR \\
\hline 15-May-97 & EI & $27342 \mathrm{G}$ & GAS STOKAGE & BWRC & PFF & $?$ & & 10 & FI & 0 & POOR & POOR \\
\hline $15-M_{n y} \cdot 97$ & E1 & $27342 \mathrm{ZH}$ & GAS STORAGE & BWHC & PFP & $?$ & 2007 & 10 & $\mathrm{PC1}$ & 0 & POOR & POOR \\
\hline 15-May-97 & E1 & $2734 \mathrm{ZJ}$ & LOQUID NITROGEN STOZRAGE TANKK̈BUILDING & BWHC & PFP & $?$ & & 10 & PC1 & 0 & POOR & POOR \\
\hline 15-May-97 & E1 & $2734 \mathrm{ZK}$ & GAS STORAGE & BWHC & PFP & $?$ & & 10 & PCI & 0 & POOR & POOR \\
\hline 15.May.97 & E1 & $277 \mathrm{~T}$ & BLOW DOWN BUILDING & RFSH & T-PLANT & 1977 & & 120 & $\mathrm{PCI}$ & 0 & MARGINAL & ACCEPTABLE \\
\hline 15-May-97 & E1 & 2828 & WATER PUMP HOUSE & BWHC & WESF & 1963 & & 21 & PC1 & 0 & POOR & POOR \\
\hline 15-May-97 & E1 & $282 \mathrm{BA}$ & WATER PUMP HOUSE & BWHC & WESF & 1968 & & 21 & $\overline{\mathrm{PCI}}$ & 0 & $\overline{\mathrm{POO}}$ & POOR \\
\hline 15-May-97 & E1 & $2901 Y$ & 100200 VALVE HOUSE & DYN & GPF & 1960 & & 55 & $\mathrm{PC1}$ & 0 & MARGINAL & POOR \\
\hline 15-May-97 & $\mathbf{E}$ & $291 \mathrm{~A}$ & AIR FILTER/STACK PLENOM & BWHC & PUREX & 7956 & & 250 & PC1 & 0 & POOR & ROOR \\
\hline 15-May-97 & El & 291AE & FILTER CELI /4 & BWHC & PUREX & 1956 & & 420 & $\mathrm{PC1}$ & 0 & POOR & POOR \\
\hline 15-May-97 & E1 & 291BA & EXHAUST AIR SAMPLE HOUSE & BWHC & B-PLANT & 1955 & & 5 & PC1 & 0 & POOR & POOR \\
\hline 15.May.97 & E1 & $291 \mathrm{BB}$ & EXKAUST AIR FLTERS, INSTRUMENTATION HOUSE & BWHC & B-PLANT & 1965 & & 13 & PCI & 0 & POOOR & FOOR \\
\hline 15-May-97 & E1 & $291 T$ & EXHAOST STACK & RFSH & T-PLANT & 1947 & & 100 & PCl & 0 & POOK & ACCEPIABLE \\
\hline 15-May-97 & E1 & $292 \mathrm{~B}$ & STACK MONITORING STATTON & BWHC & B-PLANT & 1944 & & 31 & PCI & 0 & POOK & POOR \\
\hline 15-Mny-97 & E1 & $292 \mathrm{~T}$ & FSSION PRODUCTS RELEASE LABORATORY & RFSH & T-PLANT & 1950 & & 85 & PCI & 0 & POOR & ACCEPTABLE \\
\hline 15-May-97 & E1 & $303 \mathrm{~A}$ & STORAGE BUILDING & BWHC & FFF & 1943 & & 120 & PCI & 0 & POOR & POOR \\
\hline 15.Mny-97 & EI & $303 \mathrm{~B}$ & STORAGE BUTLDING & BWHC & PF & 1943 & & 120 & PC1 & 0 & POOR & FOOK \\
\hline 15-May-97 & E1 & $303 E$ & ESSENTIAL MATERIALS STORAGEBUILDING & BWHC & FFF & 1943 & & 120 & PC1 & 0 & POOR & POOR \\
\hline 15-May-97 & E1 & $303 \mathrm{~F}$ & PUMP HOUSE & BWHC & FFF & 1943 & & 120 & PCI & 0 & POÓR & POOR \\
\hline 15.May-97 & E1 & $303 \mathrm{G}$ & ESSENTIAL MATERIAIS STORAGĒ BUILDING & BWHC & FFF & 1943 & & 120 & $\mathrm{PCI}$ & 0 & POOOR & POOR \\
\hline 15-May-97 & EI & $303 \mathrm{~K}$ & $\begin{array}{l}\text { CONTAMGNATED WASTE STOKAGE FACIUTYY, DECONTAM\& } \\
\text { BATTERY CHARGINGFACIUTY }\end{array}$ & BWHC & FFF & 7943 & & 120 & PCI & 8 & POOR & POOR \\
\hline 15-May-97 & E1 & $303 \mathrm{M}$ & URANIUM OXIDE FACIUITY & BWHC & FFF & 1983 & & 264 & $\mathrm{PC1}$ & 0 & MARGINAL & POOR \\
\hline 15-May-97 & E1 & 304 & URANIUM CONCRETION FACIUTY AND STORAGE AREA & BWHC & FFF & 1954 & & 115 & PCl & 0 & POOR & POOR \\
\hline 15-1May-97 & E1 & 308 & FUELS DEVELOPMENT LABORATORY & BWHC & FFF & 1960 & & 5667 & PCI & 0 & POOR & VERIFIABLE \\
\hline 15-Moy-97 & E1 & $308 \mathrm{~A}$ & FUELS DEVELLOPMENT LABORATOR & BWHC & FFF & 1975 & & 873 & PC1 & 0 & FOOKR & POOR \\
\hline 15-May.97 & E1 & 3135 & 313.5 & BWHC & FFF & 1943 & & 2764 & $\mathrm{PC1}$ & 0 & POOR & POOR \\
\hline 15-May-97 & E1 & $324 \mathrm{~B}$ & CHEMICAL ENGINEERING LAB̄ORATORY Y, ĒXHAUST TTACK & BWHC & FFF & 1965 & & 5 & PC1 & 0 & MARGINAL & MARGINAL \\
\hline 15-May-97 & E1 & $340 \mathrm{~A}$ & WASTE RETENTIONBUILDING 340 ANNEX & RFSH & DEF & 1953 & 1999 & 127 & FCI & $\overline{0}$ & POOR & POOR \\
\hline 15-May-97 & E1 & $340 \mathrm{~B}$ & WASTE LOADOOUT BULLDING & RFSH & LEF & 1953 & 1999 & 112 & $\mathrm{PC1}$ & 0 & POOR & POOR \\
\hline 15-May-97 & E1 & 3706 & INFÖRMATION SERVICES & DYN & GPF & 1944 & & 3485 & PC1 & 0 & POOR & \\
\hline 15-May-97 & E1 & $3707 \mathrm{~F}$ & RADIATION MONITORING BUILDING & RFSH & $\mathrm{LEF}$ & 1964 & 2017 & 13 & $\mathrm{PCI}$ & 0 & POOR & POOK \\
\hline $15 \cdot$ May-97 & $\mathrm{EI}$ & $3707 \mathrm{G}$ & CHANGEHOUSE & BWHC & FFF & 1940 & & 18 & PC1 & 0 & POOK & POOR \\
\hline 15-Mny-97 & E1 & 3711 & MAINTENANCE STORAGE BUILDING & DYN & GPF & 1974 & 2007 & 298 & $\mathrm{PC1}$ & 0 & POOR & POOR \\
\hline 15-May-97 & E1 & 3712 & STORAGE BUILDING & BWHC & FFF & 1961 & & 891 & $\mathrm{PCl}$ & 0 & POOR & POOR \\
\hline 15-May-97 & $\overline{\mathrm{E} 1}$ & 3716 & STORAGE BUIIDING & BWHC & FFF & 1938 & & 298 & PC1 & 0 & POOR & POOR \\
\hline 15-May-97 & E1 & $3718 G$ & STOKAGE BUIDDING & BWHC & FFF & 1978 & & 1219 & $\mathrm{PC1}$ & 0 & MARGINAL & ACCEPTABLE \\
\hline 15-May-97 & E1 & 3723 & SOLVENT AND ACID STORAGE BUILDING & BWHC & FFF & 1972 & & 13 & $P C 1$ & 0 & POOR & POOR \\
\hline 15-May-97 & El & 377 & LABORATORY & DYN & GPF & 1980 & & 336 & PC1 & 0 & POOR & FOOK \\
\hline 15-May-97 & E1 & $4713 \mathrm{D}$ & INTERIM MAINTENANCE AND STORAGE FACIITY & BWHC & FFTF & 1978 & & 587 & FC1 & 0 & MARGINAL & POOR \\
\hline
\end{tabular}


HNF-SD-GN-ER-507, REV 0

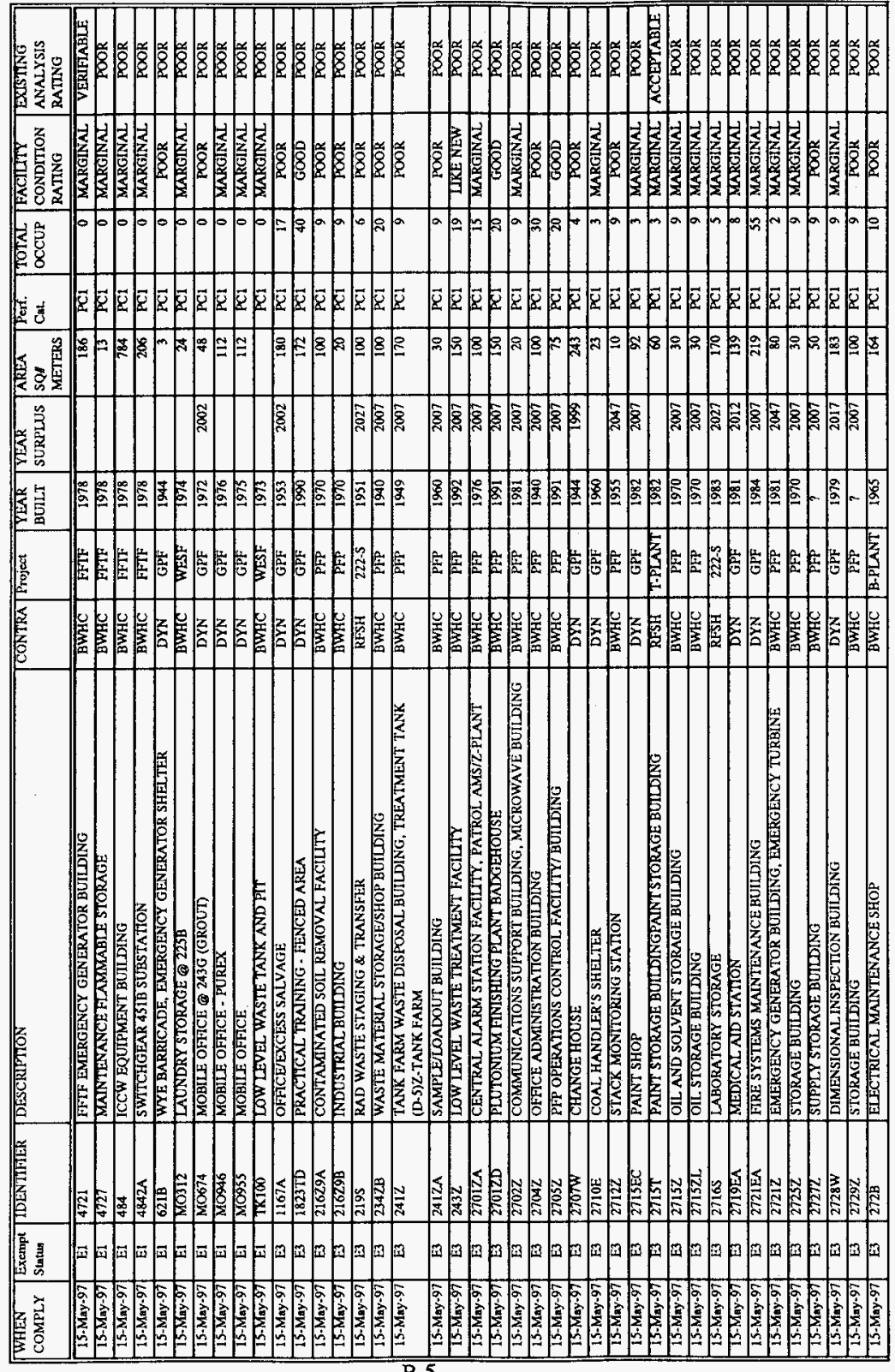




\begin{tabular}{|c|c|c|c|c|c|c|c|c|c|c|c|c|}
\hline $\begin{array}{l}\text { WTHEN } \\
\text { COMPLY }\end{array}$ & $\begin{array}{l}\text { Excanpt } \\
\text { Stahts }\end{array}$ & IDENTIFIER & DESCRIPTION & CONTRA & Project & $\begin{array}{l}\text { YEAR } \\
\text { BUILT }\end{array}$ & $\begin{array}{l}\text { YEAR } \\
\text { SURPLUS }\end{array}$ & \begin{tabular}{|l|} 
ARHA \\
SQA \\
METERS
\end{tabular} & $\begin{array}{l}\text { Perf. } \\
\text { Cat. }\end{array}$ & $\begin{array}{l}\text { TOTAL } \\
\text { OCCUP }\end{array}$ & $\begin{array}{l}\text { FACILIYY } \\
\text { CONDITON } \\
\text { RATING }\end{array}$ & $\begin{array}{l}\text { EXISTING } \\
\text { ANALYSIS } \\
\text { RATING }\end{array}$ \\
\hline 15-May-97 & $\overline{E 3}$ & 27312 & STORAGE BUILDING & BWHC & PFP & 1965 & & 100 & $\overline{\overline{P C I}}$ & $\overline{9}$ & $\overline{\text { POOR }}$ & PिOOKR \\
\hline 15-May-97 & E3 & $27312 A$ & CONTAINER STORAGE BUILDING & $B W H C$ & PFP & 1987 & & 100 & PC1 & 9 & POOR & POOR \\
\hline \begin{tabular}{|l|} 
15-May-97 \\
\end{tabular} & E3 & $27362 \mathrm{Z}$ & PLUTONUUM STORAGE VENTILATTON BUILDING/STRUCTURE & BWHC & PFP & 1977 & 2047 & 71 & PC1 & 5 & MARGINAL & VERIFIABLE \\
\hline \begin{tabular}{|l|} 
15-May-97 \\
\end{tabular} & E3 & $2736 \mathrm{ZC}$ & CARGOO RESTRAINT TRANSTOKRT DOCK & BWHC & PFP & 1980 & 2047 & 160 & $\overline{P C 1}$ & & MARGINAL & POOR \\
\hline \begin{tabular}{|l|l|}
$15-$ May-97 \\
\end{tabular} & E3 & 278AW & TANK FARM DOCUMENT CONTROL CENTER & DYN & GPF & 1994 & 2017 & 172 & $\overline{P C 1}$ & 10 & LAKE NEW & POOR \\
\hline 15-Mny-97 & E3 & $328 \mathrm{~A}$ & SHEET METAL SHOP & DYN & GPF & 1958 & 2012 & 113 & PC1 & 5 & POOR & POOR \\
\hline 15-May-97| & E3 & $3709 \mathrm{~B}$ & FIRE EQUIPMENT STORAGE BUILDING & DYN & GPF & 1977 & 2002 & 5 & $\overline{\mathrm{P}} \mathrm{C} 1$ & 15 & MARGINAL & $\overline{\mathrm{POOOR}}$ \\
\hline \begin{tabular}{|l|}
$15-$ Mny-97 \\
\end{tabular} & $\overline{E 3}$ & 3766 & OFRCE BULDING & DYN & GPF & 1975 & 2017 & 268 & PC! & 24 & MARGINAL & POOR \\
\hline 15-May-97 & $\overline{\mathrm{E3}}$ & 3768 & OFFICE BUILDING & DYN & GPF & 1976 & 2017 & 268 & PC1 & 17 & MARGINAL & POOR \\
\hline $\mid$ 15-May-97 & $\mathrm{E3}$ & 3769 & OFFICE BUILDING & DYN & GPF & 1976 & 2017 & 268 & $\mathrm{PCl}$ & 16 & MARGINAL & POOR \\
\hline 15-May-97 & E3 & 3770 & OFFCE BUIDING & DYN & GPF & 1976 & 2017 & 268 & PCI & 16 & MARGINAL & POOR \\
\hline 15-Mray-97 & E3 & 4707 & 400 AREA SITE SÚPPORT OFFICE & DYN & GPF & 1982 & 2002 & 212 & PC1 & 10 & MARGINAL & POOR \\
\hline 15-May-97 & E3 & 4716 & FFTF TOOL CRIB & BWHC & FFTF & 1978 & & 172 & $\overline{P C l}$ & 8 & MARGINAL & POOR \\
\hline 15-May-97 & E3 & 4802 & $\begin{array}{l}\text { CONSTRUCTIONSUPPORT BUIDDINGOFFICENAREHOUSE } \\
\text { BUILDING }\end{array}$ & BWHC & FFF & 1982 & 2037 & 123 & PCl & 4 & MARGINAL & FOOK \\
\hline 15.May-97 & E3 & 481 & WATER PUMP HOUSE BUILDING & BWHC & FFTF & 1978 & & 146 & $\mathrm{PCl}$ & 1 & MARGINAL & VERPLABIL \\
\hline 15.Mny-97 & $\mathrm{E}$ & $481 \mathrm{~A}$ & WATER PUMP YOUSE BUILDING & BWHC & FFTF & 1978 & & 150 & PC1 & $\mathbf{I}$ & MARGINAI & POOR \\
\hline 15-May-97 & E3 & $604 \mathrm{~F}$ & WYE BARRICADE. PATROL CHECKING STATION & DYN & GPF & 19959 & & 7 & P1 & & POOK & POOK \\
\hline 15-May-97 & $\mathbf{E 3}$ & $609 \mathrm{~B}$ & STORAGE BUILDING & DYN & GPF & 1972 & 1999 & 11 & $\overline{\mathrm{PC} 1}$ & 4 & MARGINAL & POOR \\
\hline 15-May-97 & E3 & $609 \mathrm{C}$ & FIRE DEPARTMENT TRAINING FACILITY & DYN & GPF & 1985 & 2007 & 17 & $\mathrm{PCl}$ & 37 & MARGINAL & $\overline{\mathrm{POOO}} \overline{\mathrm{R}}$ \\
\hline 15-May-97 & $\overline{\mathbf{B}}$ & 609E & STORAGE BUILDING & DYN & GPF & 1988 & 2012 & 15 & $\overline{\mathrm{PCl}}$ & 15 & $\overline{G O O D}$ & $\overline{P O O O R}$ \\
\hline 15-May-97 & E3 & $609 \mathrm{G}$ & FIRE ALARM TESTING BUIDING & DYN & GPF & 1990 & 2005 & 24 & PCI & 47 & GOOD & POOR \\
\hline 15.Mny-97 & E3 & 513 & STORAGE BUILDING & DYN & GPF & 1954 & 1999 & 14 & $\overline{\mathrm{PCI}}$ & 0 & POOR & POOR \\
\hline 15-May-97 & $\overline{E^{3}}$ & $621 \mathrm{~A}$ & YAKIMA BARRICADE EMERGE SECY GENERATOR SHELTER & $\overline{\text { DYN }}$ & $\overline{G P F}$ & 1944 & 1998 & 4 & PCI & I & POOR & $\overline{P O O K}$ \\
\hline $\mid 15 \cdot$ May-97 & $\mathbf{E B}$ & $661 \mathrm{~A}$ & TARGET RANGE CONTROL BUILDING & DYN & GPF & 1982 & 2017 & 169 & PCL & 2 & MARGINAL & POOR \\
\hline 15-May-97 & $\mathbf{E}$ & $7125 \mathrm{WIT}$ & MEDICAL DENTAL BUILOING (SUTTE 4) & DYN & GPF & 1975 & 2012 & 171 & TCI & 8 & MARGINAL & FOOR \\
\hline $15-$ May-97 & E3 & MO001 & MOBILE OFFICE @ PTA & DYN & GPF & 1974 & 2007 & 125 & $\mathrm{PCl}$ & 4 & MARGINAL & POOR \\
\hline 15-May-97 & E3 & M0002 & MOBILE OFFCE $₫$ PTA & DYN & GPF & 1974 & 2007 & 125 & FCI & 4 & MARGINAL & FOOR \\
\hline 15-May-97 & E3 & M0011 & 200W PATROL HEADQUEARTERS - TRAILER2345Z & DYN & GPF & 1977 & 2007 & 147 & $\overline{\mathrm{PCI}}$ & 6 & MARGINAL & POOR \\
\hline 15-May-97 & $\mathbf{E}$ & MOO14 & MOBILEOFFCE@2704Z & DYN & GPF & 1977 & 2007 & 86 & PCI & 6 & MARGINAL & POOR \\
\hline 15-May-97 & E3 & MO015 & MOBILE OFFICE 8 234-5Z & DYN & GPF & 1977 & 2007 & 62 & $\overline{\mathrm{PCI}}$ & 2 & MARGINAL & POOR \\
\hline 15-May-97 & B3 & MO016 & MOBILE OFFCE @ 234-5Z & DYN & GPF & 1976 & 2007 & 62 & PCI & 3 & MARGINAL & POOR \\
\hline 15-May-97 & E3 & MO017 & MOBILE OFFCE @ 234-52 & DYN & $\overline{G P F}$ & 1975 & 2007 & 62 & $\overline{P C l}$ & 4 & MARGINAL & POOR \\
\hline T5-May-97 & $\mathbf{E}$ & 100021 & MOBILE OFFCE (- 2TOM & DYN & GPF & 197 & 2007 & 147 & FCI & $\pi$ & MARGINAL & TOOR \\
\hline 15-May-97 & $\mathbf{E} \mathbf{3}$ & M0027 & MOBILE OFFICE @ 272S & $\overline{D Y N}$ & GPF & 1978 & 2007 & 172 & $\mathrm{PCl}$ & 5 & MARGINAL. & POOR \\
\hline 15-Mny-97 & E3 & M0028 & RAD WORKER TRAINING TRAILER @2704 & DYN & $\overline{G P F}$ & 1978 & 2007 & 258 & FCI & 10 & MARGINAL & POOR \\
\hline 15-Mny-97 & $\overline{E 3}$ & MO029 & MOBILE OFFCE @ 271B & BWHC & B-PLANT & 1986 & & 258 & $\overline{\mathbf{P C l}}$ & 16 & GOOD & POOR \\
\hline iS-May-97 & E3 & MO03! & MOBILE OFFICE $@ 234-52$ & DYN & GPF & 1978 & 2007 & 258 & PCI & 16 & MARGINAL & POOR \\
\hline 15-May-97 & $\bar{E}$ & $\overline{\mathrm{MO032}}$ & MOBILE OFFICE @ 234-5Z & DYN & GPF & 1976 & 2007 & 258 & PCI & 20 & MARGINAL & POOR \\
\hline 15-May-97 & E3 & MO0136 & MOBILE OFFCE (340 TRL I) & RFSH & LEF & 1983 & 1999 & 147 & FCI & 70 & MARGINAL & POOR \\
\hline 15-May-97 & E3 & MO039 & MOBILE OFFCE @ $2704 \mathrm{~S}$ & DYN & GPF & 1979 & 2007 & 258 & $\overline{\mathrm{PCI}}$ & 16 & MARGINAL & POOR \\
\hline
\end{tabular}




\begin{tabular}{|c|c|c|c|c|c|c|c|c|c|c|c|c|}
\hline $\begin{array}{l}\text { WHEN } \\
\text { COMPLY }\end{array}$ & $\begin{array}{l}\text { Excmpt } \\
\text { Statums }\end{array}$ & TDENIIFIER & DESCRIPION & CONTKA & Froject & $\begin{array}{l}\text { YEAR } \\
\text { BUILT }\end{array}$ & $\begin{array}{l}\text { YEAK } \\
\text { SURPLUS }\end{array}$ & \begin{tabular}{l|} 
AREA \\
SQ \\
METERS
\end{tabular} & $\begin{array}{l}\text { Perf. } \\
\text { Cat. }\end{array}$ & $\begin{array}{l}\text { TOTAL } \\
\text { OCCUP }\end{array}$ & $\begin{array}{l}\text { FACILIY } \\
\text { CONDITON } \\
\text { RATING }\end{array}$ & $\begin{array}{l}\text { EXISTING } \\
\text { ANALYSIS } \\
\text { RATING }\end{array}$ \\
\hline 15-May-97 & $\overline{E 3}$ & MO040 & MOBLE OFFICE 6 284E & $\overline{\text { DYN }}$ & $\overline{\text { GPF }}$ & 1979 & 2007 & 258 & PCI & 16 & MARGINAL & $\overline{\text { POOR }}$ \\
\hline 15-May-97 & $\mathbf{E}$ & MOO41 & MOBTE OFFCE $243 \mathrm{G}$ & DYN & GPF & 1979 & 2007 & 172 & PC1 & 8 & MARGINAL & POOR \\
\hline 15-May-97 & E3 & MO042 & MOBILE OFFCE @ 2750E & DYN & GPF & 1979 & 2007 & 258 & PCI & 10 & MARGINAL & FOOR \\
\hline $15-$ Msy-97 & $\overline{E 3}$ & MOOO48 & 200 AREA CENTKAL SIGN SHOP $Q 274 \mathrm{E}$ & DYN & GPF & 1979 & 2007 & 172 & PC1 & 11 & MARGINAL & POOR \\
\hline 15-May-97 & E3 & MO052 & MOBILE OFFCE (333 TRL 1) & BWHC & FFF & 1980 & 1999 & 170 & $\overline{\mathrm{FC1}}$ & 9 & MARGINAL & POOR \\
\hline 15-May-97 & E3 & MOIO1 & MOBILE OFFCE @ I7IIK (FXA I111N) & DESH & SNF & & & 147 & PC1 & 50 & GOOD & POOR \\
\hline 15-May-97 & $\overline{\mathrm{E} 3}$ & MO102 & MOBILE OFHCE TRAILER 1109N & DESH & SNF & & & 147 & PC1 & 50 & GOOD & POOR \\
\hline 15-May-97 & $\overline{E 3}$ & MÖÖB & MOBILE OFFICE $\triangle$ 4TH \& BKLTIMORE $(5 \bar{B})$ & DYN & GPF & 1983 & 2007 & 156 & RCI & 7 & MARGINAL & $\overline{\mathrm{P} O \bar{O} \mathbf{R}}$ \\
\hline 15-May-97 & $\overline{\mathrm{E}} \mathbf{3}$ & MOLOA & OFFCE/CHANGETRANING TRALER @224B & DYN & GPF & 1937 & 2002 & 172 & $\mathrm{PC1}$ & 7 & MARGINAL & POOR \\
\hline 15-May-97 & E3 & MO201 & MOBILEOFFCE $62704 \mathrm{~W}$ & DYN & OPF & 1976 & 1998 & 86 & FET & & MARGINAL & POOR \\
\hline 15-May-97 & E3 & MO203 & GRAPHICS FACIUTY @ 284E (ATT TOMO931) & DYN & GPF & 1987 & 2007 & 61 & $\mathrm{PC1}$ & 8 & MARGINAL & POOR \\
\hline 15-May-97 & E3 & MO206 & MOBILE OFHCE @ B PLANT 22SB & DYN & GPF & 1977 & 2002 & 62 & $\overline{\mathrm{PC1}}$ & 5 & MARGINAL & POOR \\
\hline |15-May-97 & $\mathbf{B}$ & MO214 & (AKA:1701K)MOBILOFFCE TRAILER & DESH & SNF & & & 170 & PC1 & 50 & GOOD & POOR \\
\hline 15-May-97 & $\mathbf{E}$ & MO222 & GUNCLEANING TRAILER @ PTA & DYN & GPF & 1977 & 2007 & 67 & PCI & 3 & MARGNAL & POOK \\
\hline $15-$ May- -97 & $\overline{E 3}$ & MO223 & CHANGE TRALER N/O 272WA & RFSH & SWM & 1982 & & 38 & PC1 & 10 & MARGINAL & POOR \\
\hline 15-May-97 & $\bar{E}$ & MO227 & MÖBIIE OFFCE $Q$ UNSECURĒD CORE AREA & DYN & GPF & 1982 & 2002 & 172 & $\overline{\mathrm{PC} 1}$ & 13 & MARGINAL & POOR \\
\hline $15-$ May- 97 & E3 & MO232 & MOBBILE OFFCE $271 \mathrm{~B}$ & BWHC & B-PLANT & 1986 & & 170 & $\mathrm{PC1}$ & 21 & MARGINAL & POOR \\
\hline $15-$ May-97 & E3 & MO236 & TELECOMMUNICATIONS SHOP (IIS4MOBIL OFFICE (1154) & DESH & SNF & & & 170 & FI & 50 & GOOD & POOR \\
\hline $15-$ May-97 & E3 & MO239 & MOBILE OFFCE & DESH & SNF & & & 170 & $\overline{\mathrm{PC} 1}$ & 50 & GOOD & POOR \\
\hline 15-May-97 & E3 & M0247 & MOBILE OFFICE - TRAILER GATE 815 & DYN & GPF & 1990 & 2007 & 172 & PC1 & To & $\overline{G O O D}$ & POOR \\
\hline $15 \cdot \mathrm{May}-97$ & E3 & MO250 & MOBILE OFFCE Q 234-5Z (OUTSLDE FENCE) & $\overline{D Y N}$ & GPF & 1990 & 2007 & 172 & PC1 & 23 & GOOD & POOR \\
\hline 15-May-97 & E3 & MO251 & MOBILE OFFICE, - TRAILER GATE 850 & DYN & GPF & $19 \times 0$ & 2007 & 172 & PC1 & 12 & अणО & POOR \\
\hline 15-May-97 & E3 & MO254 & MOBILE OFFICE @ 622G & DYN & GPF & 1990 & 2007 & 172 & PC1 & II & GOOD & FOOR \\
\hline 15-May-97 & $\overline{E 3}$ & MO255 & MOHIIE OFFICE Q 622G & $\overline{D Y N}$ & GPF & 1990 & 2007 & 172 & $\overline{\mathrm{PCI}}$ & 14 & $\overline{G O O D D}$ & POOOR \\
\hline $15-\mathrm{M} \Delta y-97$ & $\mathbf{E X}$ & MO256 & MOBILE OFFICE E/O 27IIE & DYN & GPF & 1990 & 2012 & $\sqrt{72}$ & PC1 & II & GOOD & FOOR \\
\hline $15-$ May-97 & $\mathrm{E}^{3}$ & MO257 & MOBILE OFFICE JO 27IIE & DYN & GPF & 1990 & 2012 & 172 & FI & III & GOOD & POOR \\
\hline 15-May-97 & E3 & MO258 & MOBILE OFFCE Q FMI & DYN & GPF & 1990 & 2017 & 172 & PC1 & 15 & GODD & PODR \\
\hline 15-May-97 & E3 & MO259 & MOBILE OFFICE \& FMT & DYN & GPF & 1990 & 2017 & 172 & $\mathrm{FCl}$ & 15 & GOOD & POOR \\
\hline 15.May.97 & $\mathbf{E}$ & MO262 & MOBILE OFFICE @ FMT & DYN & GPF & 1990 & 2017 & 172 & PCI & 15 & GOOD & POOR \\
\hline 15-May-97 & E3 & MO263 & MOBILE OFFCE @ FMIT & DYN & GPF & 1990 & 2017 & Th2 & PCI & 15 & GOOD & POOR \\
\hline 15-May-97 & E3 & MO264 & MOBILE OFFCE @ FMT & DYN & GPF & 1990 & 2017 & 172 & $\overline{\mathrm{PCl}}$ & 15 & GOOD & POOR \\
\hline IS-May-97 & E3 & MO266 & MOBILE OFFICE @ 272AW TANK FARMMS & DYN & $\overline{\mathrm{GPF}}$ & 1990 & 2007 & 172 & PCI & 16 & $\overline{\mathrm{GOOD}}$ & POOR \\
\hline 15-May-97 & E3 & MO267 & MOBILE OFFCE @ 272AW TANK FARMS & DYN & GPF & 1990 & 2007 & 172 & PC1 & 16 & GOOD & POOR \\
\hline 15.May.97 & $\mathbf{E}$ & MO268 & MOBLE OFFCE @ 272AW TANK FARMS & DYN & GPF & 1990 & 2007 & $17 \sqrt{2}$ & PCI & 16 & GOOD & POOKR \\
\hline 15-May-97 & E3 & MO270 & MOBILE OFFCE (3765T) & BWHC & FFF & 1991 & 2001 & 172 & PCI & 20 & GOOD & ACCEPTABLE \\
\hline 15-May-97 & E3 & MO271 & MOBILE OFFICE (3763Ti) & BWHC & FFF & 1941 & 2001 & 172 & PCI & 20 & $\overline{\mathrm{GOO}} \overline{\mathrm{D}}$ & ACCEPTAB̈LE \\
\hline 15-May-97 & E3 & MO275 & MOMILE OFFCE (324T2) & BWHC & FFF & 1991 & 2001 & 170 & $\mathrm{PCl}$ & 20 & GOOD & ACCERTABLE \\
\hline 15.May-97 & $\mathbf{E 3}$ & MO282 & MOBILE OFFICE @ GROUT & DYN & GPF & 1993 & 2007 & 172 & PCI & 18 & DKENEW & POOR \\
\hline 15. May-97 & E3 & $\mathrm{MO} 283$ & MOBILE OFFICE @ GROUT & DYN & GPF & 1993 & 2007 & 172 & $\mathrm{PC1}$ & 18 & LIKE NEW & FOOR \\
\hline 15.Mny-97 & $\mathbf{E}$ & MO284 & MOBILE OFFICE Q GROUT & DYN & GPF & 1993 & 2007 & 172 & $\mathrm{PC1}$ & 18 & UIRE NEW & POD \\
\hline $15-\mathrm{May}-97$ & E3 & MO302 & ARMORER SHOP (MOBILE OFFCE) QPTA & DYN & GPF & 1974 & 2007 & I112. & $\mathrm{PCl}$ & 3 & MARGINAL & FOOR \\
\hline
\end{tabular}




\begin{tabular}{|c|c|c|c|c|c|c|c|c|c|c|c|c|}
\hline $\begin{array}{l}\text { WHEN } \\
\text { COMPLY }\end{array}$ & $\begin{array}{l}\text { Excmpt } \\
\text { Status }\end{array}$ & IDENTHER & DESCRIPTION & COANTRA & Froject & $\begin{array}{l}\text { YEAR } \\
\text { BUILT }\end{array}$ & \begin{tabular}{|l|} 
YEAR \\
SURPLUS
\end{tabular} & \begin{tabular}{|l|} 
AREA \\
SQ \\
METERS
\end{tabular} & $\begin{array}{l}\text { Perf. } \\
\text { Cat. }\end{array}$ & $\begin{array}{l}\text { TOTAL } \\
\text { OCCUP }\end{array}$ & $\begin{array}{l}\text { FACILITY } \\
\text { CONDITION } \\
\text { RATING }\end{array}$ & $\begin{array}{l}\text { EXISTING } \\
\text { ANALYSIS } \\
\text { RATING }\end{array}$ \\
\hline 15-May-97 & $\bar{E}$ & $\overline{M 0303}$ & MOBILE OFFCE W. OFII67A & $\overline{\text { DYN }}$ & $\overline{\mathrm{GPF}}$ & 1975 & 1998 & 112 & PC1 & 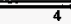 & MARGINAL & FOOR \\
\hline $15-$ May-97 & E3 & M0346 & MOBICEOFFICE 2225 & DYN & GPF & 1977 & 2007 & II2 & FC1 & 11 & MARGINAL & POOR \\
\hline \begin{tabular}{|l|}
$15-M a y-97$ \\
\end{tabular} & E3 & MO347 & MOBLE OFFCE 202 A (ATT'D TO MO948) & DYN & GPF & 1975 & 2002 & 120 & $\overline{\mathrm{PC} 1}$ & 6 & POOR & POOR \\
\hline 15-May-97 & E3 & MO354 & MOBILE OFFICE $2400 \mathrm{E}$ & DYN & GPF & 1974 & 2002 & 120 & $\mathrm{PCl}$ & 8 & POOR & POOR \\
\hline 15.May-97 & E3 & M0355 & MOBILE OFHCE $(202$ (ATTD TO MOS42) & DYN & GPF & 1974 & 2002 & 120 & PCI & 8 & POOR & POOR \\
\hline 15-May-97 & E3 & MO368 & PATROL TRAINING (MOBILE OFFCE) QPIA & DYN & GPF & 1975 & 2007 & 112 & FCI & 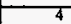 & MARGINAL & POOK \\
\hline \begin{tabular}{|l|}
$15-$ May-97 \\
\end{tabular} & E3 & MO382 & MOBILE OFFCE TRALER - $1721 \mathrm{~K}$ & DESH & SNF & & & 170 & PCI & 50 & GOOD & POOR \\
\hline \begin{tabular}{|l|}
$15 \cdot M a y \cdot 97$ \\
\end{tabular} & $\mathbf{B}$ & MO386 & MOBILE OFFICE @ DMRHF (24006) & DYN & $\overline{G P F}$ & 1986 & 2007 & $\overline{172}$ & PCI & 9 & MARGINAL & PODK \\
\hline \begin{tabular}{|l|}
$15-M a y-97$ \\
\end{tabular} & E3 & MO388 & SRT HEADQUARTERS (2721EA) & DYN & $\overline{G P F}$ & 1989 & 2002 & 12 & PCI & 16 & GOOD & POOR \\
\hline 15-May-97 & E3 & M0393 & MOBILE OFFCE ( 272AWTAMK FARMS & DYN & GPF & 1974 & 2002 & 112 & PC1 & 10 & MARGINAL & POOR \\
\hline 15-May-97 & $\mathbf{E} 3$ & MO401 & MOHILE OFFCE @ 1719K (FKA: 1117N) & DESH & SNF & & & 170 & $\overline{\mathrm{PCI}}$ & 50 & GOOD & POOR \\
\hline \begin{tabular}{|l|}
$15 \cdot$ May-97 \\
\end{tabular} & E3 & MO402 & MOBILE OFFCE TRAILER 1718K & DESH & SNF & & & 170 & $\overline{\mathrm{PCI}}$ & उ0 & GÓOD & $\overline{\text { POŌŔ }}$ \\
\hline $15 \cdot$ May.97 & $\overline{E 3}$ & MO408 & MOBILE OFFCE 9 271B & $\overline{D Y N}$ & GPF & 1983 & 2002 & 258 & $\overline{\mathrm{PCl}}$ & 17 & MARGINAL & POOR \\
\hline \begin{tabular}{|l|}
$15-$ May-97 \\
\end{tabular} & E3 & MOAIO & MOBIL OFFCE 92718 & BWHC & B.PLANT & 1986 & & 250 & RC1 & 16 & GOOD & FOK \\
\hline 15-May-97 & $\bar{E}$ & MO420 & MOBILE OFFICE (PIPEYARD) & DESH & SNF & & & 170 & $\overline{\mathrm{PCl}}$ & 50 & GOOD & POOR \\
\hline \begin{tabular}{|l|}
$15 \cdot$ May $\cdot 97$ \\
\end{tabular} & $\mathbf{E 3}$ & MO428 & MOHLLE FIELD OFFCE @ LERF & BWHC & PFP & 1990 & 2007 & 130 & $\overline{\mathrm{PCI}}$ & 30 & GOOD & POOR \\
\hline 15-May-97 & $\bar{E}$ & MO429 & MOBILE FIELD OFFCE @ IERF & BWHC & $\overline{\text { PFP }}$ & 1990 & 2007 & 130 & $\overline{\mathrm{PCI}}$ & 50 & GOODD & POOR \\
\hline 15-May-97 & E3 & MO433 & MOBILE OFHCECHANGE OFHICE (8 271TMOBILE OFFICE (27T1) & RFFH & T-PLANT & 1994 & 2017 & 180 & PCT & $\frac{12}{12}$ & DKENNEW & ACCEPTABLE \\
\hline 15-May-97 & $\mathbf{E} \mathbf{3}$ & MO434 & MOBILE OFFCE @ 209E & DYN & GPF & 1993 & 20007 & 172 & PCI & 12 & LIKE NEW & FOOOR \\
\hline 15-May-97 & $\bar{E}$ & MO437 & MOBILE OFFICE 272WA & RFSH & SWM & 1995 & & 172 & $\overline{P C 1}$ & 25 & LKRE NEW & MARGINAL \\
\hline $15-\mathrm{May}-97$ & $\mathbf{E 3}$ & MO438 & MOBLE OFFICE 272WA & RFSH & SWM & 1995 & & Th2 & PCI & 32 & TKENEW & MARGINAI \\
\hline $15-$ May-97 & $\mathbf{E}$ & MO442 & MOBILE OFFICE & DESH & SNF & & & 170 & FCI & 30 & GOOD & POOR \\
\hline 15-May-97 & E3 & MO459 & WOMEN'S CHANGE TRAILER & RFSH & T.PLANT & 1996 & & & PC1 & 12 & LIKE NEW & ACCEFTABLE \\
\hline $15-$ May-97 & $\bar{E}$ & MO535 & MOBIIE OFFICE (1155N) & RFFH & SWM & 1978 & & 120 & $\overline{\mathrm{PCT}}$ & $\bar{T}$ & MAR̆GINAL & MARGINAL \\
\hline 15-May-97 & $\bar{E}$ & MO536 & MOBILE STORAGE (1129N) & DYN & $\overline{G P F}$ & 1979 & 2007 & 120 & $\overline{\mathrm{PCI}}$ & 5 & MARGINAL & $\overline{P O O R}$ \\
\hline $15-$ May-97 & $\mathbf{E}^{3}$ & MO\$42 & MOBILE OFFICE @ 202A (ATT'D/LD'DMO355) & DYN & GPF & 1974 & 2002 & T20 & PCI & II & MARGINAL & POOR \\
\hline 15-May-97 & E3 & MO560 & MOBILE OFFCE @ UNSECURED CORE AREA & DYN & GPF & 1979 & 2002 & 156 & PCI & 8 & MARGINAL & POOR \\
\hline 15-May-97 & E3 & MO656 & MOBILE OFFCE TRAILER & DYN & GPF & 1967 & 2007 & 9 & PCI & 4 & MARGINAL & POOR \\
\hline 15-May-97 & E3 & MO744 & MOBILE OFFCE $\odot$ TEDF & RFSH & IEF & 1994 & 2007 & 172 & PCI & 15 & UKE NEW & FOOR \\
\hline 15-May-97 & $\mathbf{E}^{3}$ & MO745 & MOHIL OFFCE S TLDF & RFSH & DEF & 1994 & 2007 & 772 & PCI & 13 & UKENEW & POOR \\
\hline 15-May-97 & E3 & MO834 & MOBILE OFFCE & BWHC & PFP & 1970 & 2007 & 70 & PC1 & 9 & MARGINAL. & POOR \\
\hline 15-May-97 & E3 & MO838 & MOBILE OFFCE @ 4TH \& BALTMORE & DYN & GPF & 1978 & 2002 & i12 & PC1 & 13 & FOOK & FOOR \\
\hline 15-May-97 & $\overline{\mathrm{E} 3}$ & MO839 & MÖBILE @ ZPLANT & BWHC & PFP & 1970 & 2007 & 70 & PC1 & $\overline{9}$ & MARGINAL & $\overline{\mathrm{POOK}}$ \\
\hline $15-\mathrm{M} a \mathrm{y}-97$ & E3 & MO863 & OFFCELDUNCHROOM TRAILER $@ 225 \mathrm{~B}$ & BWHC & WESF & 1982 & 2017 & 147 & PC1 & 8 & MARGINAL & POOR \\
\hline 15-May-97 & E3 & MO89? & MOBILE OFFCE W/O 27TT & RFSH & T-PIANT & 1977 & 2007 & 125 & PC1 & 31 & MĀRGINAL & ACCEPTABLE \\
\hline 15-May-97 & E3 & MO907 & OFFCE TRAILER @ 1722K MOBILE OFFCE TRAILER $1722 \mathrm{~K}$ & DESH & SNF & & & 170 & PCI & 50 & GOOD & POOR \\
\hline 15-May-97 & E3 & M0922 & OFFCECHANGE TRALLER Q 2703E & DYN & GPF & 1977 & 2002 & 125 & PC1 & 10 & MARGINAL & POOR \\
\hline $15 \cdot-4 \mathrm{ny}-97$ & E3 & MO923 & MOBILE OFHCE (1126N) & DESH & SNF & & & 170 & RI & 50 & GOOD & POOK \\
\hline 15-May-97 & E3 & $\overline{M 0924}$ & MOBILE OFFICE $202 \mathrm{~S}$ & DYN & GPF & 1977 & 2007 & 125 & PCI & 10 & MARGINAL & POOR \\
\hline 15.Mny-97 & $\mathrm{E}_{3}$ & MO928 & MOBILE OFFCE @ 1723K (FKA II23N) & DESH & SNF & & & 170 & PC1 & 50 & GŌDD & FOOR \\
\hline $15-$ May-97 & Ej & MO931 & CHANGEJLUNCHEROOOM $(284$ E) & $\overline{D Y N}$ & $\overline{\mathrm{GPF}}$ & $\overline{9795}$ & 2002 & 133 & $\overline{P C 1}$ & 9 & MARGINAL & $\overline{P O O} \overline{\mathrm{R}}$ \\
\hline
\end{tabular}




\begin{tabular}{|c|c|c|c|c|c|c|c|c|c|c|c|c|}
\hline $\begin{array}{l}\text { WHEN } \\
\text { COMTLY }\end{array}$ & $\begin{array}{l}\text { Exempt } \\
\text { Staturs }\end{array}$ & IDENTIFIER & TDESCRIPIION & CONTRA & Project & $\begin{array}{l}\text { YEAR } \\
\text { BUILT }\end{array}$ & $\begin{array}{l}\text { PEAR } \\
\text { SURPLUS }\end{array}$ & \begin{tabular}{|l} 
AREA \\
SQ \\
METERS
\end{tabular} & $\begin{array}{l}\text { Perf. } \\
\text { Cat. }\end{array}$ & TOLAL & $\begin{array}{l}\text { FACILITY } \\
\text { CONDITION } \\
\text { RATING }\end{array}$ & $\begin{array}{l}\text { EXISTING } \\
\text { ANALYSIS } \\
\text { RATING }\end{array}$ \\
\hline 15-May-97 & $\overline{E \text { E3 }}$ & MO936 & MOBILE OFFICE (222S) & DYN & $\overline{\mathrm{GPF}}$ & 1977 & 2007 & 147 & $\overline{\mathrm{PC1}}$ & 5 & MARGINAL & FOOR \\
\hline 15-Mny-97| & E3 & MO939 & MOBILE OFFICE (2345Z) & DYN & GPF & 197 & 2007 & 62 & PCI & 4 & MARGINAL & POOK \\
\hline 15-May-97 & E3 & MO947 & MOBILE OFFICE @ 2704S & $\overline{\text { DYN }}$ & GPF & 1976 & 2007 & 112 & PC1 & 0 & MARGINAL & POOR \\
\hline 15-May-97 & E3 & MO948 & MOBILE OFFICE - $202 \mathrm{~A}$ & DYN & GPF & 1975 & 2002 & 120 & PC1 & 6 & FOOR & POOK \\
\hline 15-Mky-97 & E3 & MO969 & MOBILE OFFICE MOBILE OFFICE & DESH & SNF & & & 170 & $\overline{\mathrm{PCl}}$ & 50 & GOOD & POOR \\
\hline $15-1,6 y-97$ & E3 & MO991 & MOBILE OFFCE - TRAILER CORE AREA & DYN & GPF & 1975 & 2002 & 172 & PCI & 12 & MAIRGINAL & POOR \\
\hline 15.Mey-97 & E3 & MO994 & MOBLLE OFHCE AT MO227/560 & DYN & GPF & 1975 & 2002 & 125 & $\overline{\text { PC1 }}$ & 6 & MARGINAL & POOR \\
\hline 15-Mny-97 & 83 & MŌ95 & MOBLE OFFICE & BWFC & B-PLANT & 1986 & & 125 & PCI & 80 & GOOD & POOR \\
\hline 15-Mey-97 & $E 3$ & MO996 & MOBLLE OFFCE (272AW) & DYN & GPF & 1975 & 2002 & 125 & FCI & 14 & MARGINAL & POOR \\
\hline \begin{tabular}{|c|}
$15-$ May-97 \\
\end{tabular} & F3 & MOS97 & MOBILE OFFICE @ 2A3G (GROUT) & DYN & GPF & 1975 & 2002 & 125 & PCI & 7 & TMARGINAL & POOR \\
\hline 15-Mny-97 & ES & 1979SNYDER & 1979 SNYDER & DYN & GPF & 1994 & 2017 & 1632 & PC1 & 56 & LKE NEW & POOR \\
\hline 15-Mny-97 & E5 & $2025 \mathrm{E}$ & PUREX LQUID EFFLUENT TREATMENT FACILTYY & RFSH & LEF & 1994 & 2023 & 3717 & Fi & 30 & LIKE NEW & POOR \\
\hline 15-May-97 & ES & 2025EA & EFFLUENT TREATMENT OPERATON SUPPORT FACILTY & RFSH & LEF & 1994 & 2025 & 1208 & PC1 & 60 & LIRE NEW & POOR \\
\hline 15-May-97 & E5 & 222SA & CHEMMLAL STANDATWSTRROCESSDEV LABORATORY & RFSH & $222-5$ & T981 & 2027 & 410 & $\mathrm{PCT}$ & 15 & MAKGINAL & ROOK \\
\hline 15-M^y.97 & ES & 2261STEVENS & OFFCE BUILDING & DYN & GPF & 1994 & $20 ! 7$ & 4132 & $\overline{\mathrm{PC} 1}$ & 220 & LUKE NEW & POOR \\
\hline 15-May-97 & ES & $2336 \mathrm{~W}$ & $\begin{array}{l}\text { WASTE RECEVING AND PROCESSINGFACIIITYWASTE } \\
\text { RECEVING AND PROCESSING }\end{array}$ & RFSH & WRAP & 1996 & 2027 & 3000 & $\overline{\mathrm{FCl}}$ & 36 & LKE NEW & ACCEPTABIIE \\
\hline \begin{tabular}{|l|}
$15-$ May-97 \\
\end{tabular} & ES & $234.52 \mathrm{~A}$ & PFP CHANGE ROOM ADDITION & BWHC & PFP & 1992 & 2007 & 430 & PCI & 30 & GOOD & POOK \\
\hline 15.Mny-97| & $\mathrm{ES}$ & $2420 \mathrm{STVCN}$ & 2420 STEVENS & DYN & GPF & 1995 & 2022 & 8709 & $\mathrm{PCl}$ & 445 & LIKE NEW & POOR \\
\hline 15-May-97 & ES & 2425STVCN & 2425 STEVENS CENTER PLACE & DYN & GPF & 1990 & 2022 & 4132 & $\overline{\mathrm{PCl}}$ & 220 & GODD & FOOK \\
\hline 15-May-97 & ES & 2430STVCN & 2430 STEVENS CENTER PLACE & DYN & GPF & 1995 & 2022 & 416 & $\overline{\mathrm{PCl}}$ & 185 & LKE NEW & POOOR \\
\hline 15-May-97 & 75 & 2440STVCN & 2440 STEVENS CENTER PLACE & DYN & GPF & 1990 & 2022 & 9182 & PCI & 475 & GOOD & POOR \\
\hline 15-May-97 & ES & 2702 & PFP OPERATIONS AND SUPPORT FACIUTY & BWHC & PFP & 1988 & & 1200 & $\mathrm{PCl}$ & 101 & GOOD & POOR \\
\hline \begin{tabular}{|l|}
$15-M a y-97$ \\
\end{tabular} & E5 & $2727 \mathrm{E}$ & SAFEGUARD AND SECURITY OFFICE & DYN & $\mathrm{GPF}$ & 1985 & 2017 & 1766 & $\overline{\mathrm{PCI}}$ & 34 & MARGINAL & $\overline{P O O \bar{R}}$ \\
\hline \begin{tabular}{|c|}
$15-$ May-97 \\
\end{tabular} & ES & $2728 \mathrm{E}$ & FUMP STORAGË & DYN & GPF & 1985 & & 1766 & $\mathrm{PCI}$ & 34 & \multicolumn{2}{|c|}{ MARGINAL } \\
\hline 15.May-97 & E5 & $274 A W$ & OFFCE BUIDDING & DY'N & GPF & 1994 & 2017 & 836 & FCI & 37 & DKENEW & FOKR \\
\hline $15-$ May-97 & E5 & $2750 \mathrm{E}$ & WASTE MANAGEMENT SURV. AND OPERATING FACILITY & DYN & GPF & 1980 & 2017 & 8922 & $\mathrm{PCl}$ & 532 & GOOD & POOR \\
\hline \begin{tabular}{|c|}
$15-$ May-97 \\
\end{tabular} & ES & $2751 \mathrm{E}$ & OFFCE FACIUTY & DYN & GPF & 1985 & 2007 & 1394 & PCI & 83 & MARGINAL & POOR \\
\hline \begin{tabular}{|c|}
$15-$ May-97 \\
\end{tabular} & ES & 2752E & 200 EAST OFFCE & DYN & GPF & 1985 & 2007 & 1394 & $\overline{\mathrm{PCI}}$ & 69 & MARGINAL & POŌ̄ \\
\hline 15.May-97| & $\mathbf{E 5}$ & $2753 \mathrm{E}$ & OAFCE BUIDDING W/O 2750E & DYN & GPF & 1986 & 2007 & 1394 & PCT & 61 & GOOD & POOR \\
\hline \begin{tabular}{|l|}
$15-\mathrm{M} a y-97$ \\
\end{tabular} & ES & $3707 \mathrm{D}$ & INFORMATION SERVICES BUILDING & DYN & GPF & 1959 & 2007 & 797 & $\overline{\mathrm{PCI}}$ & 25 & POOR & POOR \\
\hline 15-May-97 & ES & 3790 & SECURITY OFFCE BUILDING & DYN & GPF & 1981 & 2027 & 1747 & PCI & 73 & MARGINAL & POOR \\
\hline 15-May-97 & $\overline{E S}$ & 4706 & OFFCE BUI DING & $\overline{D Y N}$ & GPF & 1983 & 2017 & 1735 & PCI & 92 & MARGINAL & POOR \\
\hline $15-\mathrm{M} z \mathrm{y}+97$ & ES & 4710 & FFIFOFFICE BUILDING & BWHC & FPFF & 1980 & & 3434 & KI & 210 & MARGINAL & FOK \\
\hline $15-\mathrm{M} a y-97$ & E5 & 4790 & PATROL HEADQUARTERS BUILDING & DYN & GPF & 1930 & 2017 & 391 & $\overline{\mathrm{PCI}}$ & 13 & MARGINAL & POOR \\
\hline \begin{tabular}{|l|} 
15-May-97 \\
\end{tabular} & E5 & 609D & TRAINING TOWER FACILTY & DYN & GPF & 1985 & 2007 & 451 & FC1 & 40 & MARGINAL & POOR \\
\hline $15 \cdot$ May-97 & E5 & 6266 & WASTE SAMPUNG AND CHARACTERLATION FACIUTY & RFSH & HAS & 1993 & 2020 & 966 & $\overline{\mathrm{P}} \overline{\mathrm{Cl}}$ & 45 & LIKE NEW & VERIFIED \\
\hline 15-May-97 & E5 & 6250 & CRANE \& RIGGING BUIDING & DYN & GPF & 1990 & 2017 & $7 \pi$ & PCI & 10 & GODD & POOR \\
\hline 15-May-97 & ES & 662 & PATROL TRAINING BUILDING \& PTA & DYN & GPF & 1982 & 2017 & 335 & PCl & 10 & MARGINAL & POOKR \\
\hline \begin{tabular}{|c|}
$5-M 9 y-97$ \\
\end{tabular} & E5 & MO037 & MÖLL & DYN & GPF & 1979 & 2007 & 315 & PCI & 33 & MARGINAL & POOR \\
\hline 15-May-97 & E5 & MO043 & MOBILE OFFCE (DOSIMETRY) @ 2750E & DYN & GPF & 1979 & 2007 & 343 & PCI & 16 & MARGINAL & FOOR \\
\hline
\end{tabular}




\begin{tabular}{|c|c|c|c|c|c|c|c|c|c|c|c|c|}
\hline $\begin{array}{l}\text { WHEN } \\
\text { COMPLY }\end{array}$ & $\begin{array}{l}\text { Exempt } \\
\text { Status }\end{array}$ & IDENTIFIER & DESCRIPTON & CONTRA & Project & $\begin{array}{l}\text { YEAR } \\
\text { BUILT }\end{array}$ & $\begin{array}{l}\text { YEAR } \\
\text { SURPLUS }\end{array}$ & \begin{tabular}{|l|} 
AREA \\
SQ" \\
MEIERS
\end{tabular} & $\begin{array}{l}\text { Perf. } \\
\text { Cat. }\end{array}$ & $\begin{array}{l}\text { TOTAL } \\
\text { OCCUP }\end{array}$ & $\begin{array}{l}\text { FACIITY } \\
\text { CONDITION } \\
\text { RATING }\end{array}$ & $\begin{array}{l}\text { EXISHING } \\
\text { ANALYYSIS } \\
\text { RATING }\end{array}$ \\
\hline 15-May-97 & $\overline{\mathrm{ES}^{5}}$ & M0047 & MOBILE OFFICE Q 2101M & $\overline{\text { DYN }}$ & GPF & 1981 & 2007 & 1116 & $\overline{\overline{P C I}}$ & 65 & MARGINAL & POOR \\
\hline \begin{tabular}{|l|}
$15-$ May-97 \\
\end{tabular} & ES & MO234 & MOBILE OFFCE @ 2750E & DYN & GPF & 1986 & 2007 & 859 & PC1 & 46 & GOOD & POOR \\
\hline \begin{tabular}{|l|}
$15-$ May-97 \\
\end{tabular} & $\overline{\mathrm{ES}}$ & MO276 & MOBILE OFFICE $\bigcirc 2753 \mathrm{E}$ & $\overline{D Y N}$ & GPF & 1992 & 2007 & 1374 & PC1 & 59 & LIKE NEW & POOR \\
\hline \begin{tabular}{|l|}
15. May.97 \\
\end{tabular} & ES & MO277 & MOBILE OFFCE $92753 \mathrm{E}$ & DYN & GPF & 1992 & 2007 & 1374 & PCI & 93 & DIKENEW & POOR \\
\hline 15-May-97 & E5 & MO278 & MOBILE OFFICE W/O $275 W$ MOBILE OFFTCE & RFSH & SWM & 1995 & & 859 & $\mathrm{PCl}$ & 60 & LIKE NEW & MARGINAL \\
\hline 15-May-97 & $\mathbf{E 5}$ & MO279 & MOBILE OFFICE W/O 275W & RFSH & SWM & 1995 & & 859 & PCl & 6 & LIKENEW & MARGINAL \\
\hline 15-May-97| & E5 & MO280 & MOBILE OFFCE @ WSCF SITE & DYN & GPF & 1992 & 2007 & 859 & $\mathrm{PCl}$ & 50 & GOOD & POOR \\
\hline 15-May-97 & E5 & MÖ281 & MOBILEOFFCE @ 272WA & DYN & GPF & 1992 & 2022 & 1374 & $\overline{\mathrm{PCl}}$ & 88 & LIRE NEW & POOR \\
\hline 15-May-97| & E5 & MO285 & MOBILE OFFCE @ 2753E & DYN & GPF & 1994 & 2007 & 1030 & PCI & 82 & LKE NEW & POOR \\
\hline 15.May.97| & E5 & MO287 & MOHIL OFPCE Q 275W & DYN & GPF & 1993 & 2007 & 1030 & TC1 & 45 & DIKENEW & FOOR \\
\hline 15-May-97 & E5 & MO291 & MOBILE OFFICE NEAR $2704 \mathrm{~S}$ & DYN & GPF & 1994 & 2007 & 859 & PCl & 65 & LIKE NEW & POOR \\
\hline 15-May-97| & $\overline{E 5}$ & MO292 & MOBILE OFFCE & DYN & GPF & 1994 & 1999 & 859 & PCI & 50 & LIKE NEW & POOR \\
\hline 15-May-97 & E5 & MO406 & CHANGEILNCHROOM TRAILER @2724WB & DYN & GPF & 1982 & $200 \overline{7}$ & 258 & $\overline{\mathrm{PCI}}$ & 5 & MARGINAL & POOR \\
\hline 15-May-97 & E5 & MO407 & MOBLLE OFFLE @ 2101M & DYN & GPF & 1983 & 2007 & 429 & $\mathrm{PCI}$ & 23 & MARGINAL & POOR \\
\hline 15-May-97 & $\overline{E S}$ & MO413 & MOBILE OFFCE $@ 2750 \mathrm{E}$ & DYN & GPF & 1984 & 2007 & 687 & PCI & 14 & MARGINAL & FOOR \\
\hline 15-May-97| & E5 & MO720 & MOBILE OFFCE $Q$ 272WA & RFSH & sWM & 1994 & & 1347 & $\mathrm{PCI}$ & 113 & LKE NEW & MARGINAL \\
\hline 15-May-97 & ES & MO721 & MOBILE CHANGE FACILTY $\Theta 272 \mathrm{WA}_{\mathrm{A}}$ & RFSH & SWM & 1994 & & 343 & PCl & 30 & UKE NEW & MARGINAL \\
\hline 15-May-97 & E5 & MO743 & MOBIDE SHOFOFFICE @ 272WA & RFSH & $S W M$ & 1994 & 2014 & S15 & PCI & 25 & UKE NEW & KARGINAL \\
\hline 15-May-97| & E\& & 1167 & STORAGE BUILDING & DYN & GPF & 1953 & 2002 & 3346 & $\mathrm{PCI}$ & 56 & POOR & POOR \\
\hline 15-May-97 & E8 & 1168 & CYLINDER STORAGE BLDG & DYN & GPF & 1953 & 2002 & 520 & $\mathrm{PCl}$ & 3 & POOKR & POOR \\
\hline 15-May-97 & $E 8$ & $1706 \mathrm{KE}$ & WATER STUDIES SEMIWORKS FACILITY & DESH & SNF & 1955 & 2002 & 1005 & PCI & & MARGINAL & POOR \\
\hline 15-May-97| & E8 & $1717 \mathrm{~K}$ & TMAINIENANCETRANSPORTATIONSHOP & DESH & SNF & 1955 & 2002 & 992 & PCI & 50 & GOOD & POOR \\
\hline 15-May-97 & E8 & $190 \mathrm{KE}$ & HGG BAY STORAGE AREA & DESH & SNF & 1955 & 2002 & 2400 & PCI & $\mathbf{I}$ & MARGINAL & POOR \\
\hline 15-May-97 & ES & $2704 \mathrm{~W}$ & OFFICE BUILDING & DYN & GPF & 1944 & 1998 & 600 & PCI & 22 & POOR & POOR \\
\hline 15-May-97 & $E 8$ & $2723 \mathrm{~W}$ & MASK LAUNDRY AND OFFCE BUILDING & DYN & GPF & 1944 & 1999 & 420 & PCI & 9 & POOK & POOR \\
\hline 15-May-97 & Es & $27 S U R$ & CHEMICAL STORAGE WAREHOUSE & DYN & GPF & 1952 & 2002 & 393 & PCI & 2 & POOR & POOR \\
\hline 15-May-97 & E8 & $313 \mathrm{~N}$ & $313-\mathbf{N}$ & BWHC & FFF & 1960 & 1999 & 4258 & PCI & 25 & POOR & POOR \\
\hline 15-May-97 & E8 & 333 & N FUELS BUILOING Chromium Trentrnent Tanks (2 Tanke) & BWHC & FFF & $i \overline{6}$ & 1999 & 5854 & $\overline{\mathrm{PC}}$ & 25 & POOR & POOR \\
\hline 15-May-97 & E8 & 340 & RETENTION AND NEUTRAIIZAITON & RFSH & LEF & 1952 & 1999 & 416 & PCI & 2 & POOR & POOR \\
\hline 15.May-97 & E8 & 4702 & OFFCE BUILDINGOFFCE BUILDING & DYN & GPF & 1948 & 2002 & 1952 & PC1 & 2 & MARGINAL & POOR \\
\hline \begin{tabular}{|c|}
$15-$ May-97 \\
\end{tabular} & E8 & $622 \mathrm{G}$ & ATMOSPHERE SCIENCE ANNEX & $\overline{D Y N}$ & GPF & 1944 & 1999 & 348 & PCI & 14 & POOR & POOR \\
\hline
\end{tabular}


HNF-SD-GN-ER-507, REV 0

\section{APPENDIX C \\ COMPLIANCE BASELINE AND PRIORITIZATION SCHEDULE FOR EXISTING BUILDINGS AND STRUCTURES REQUIRING \\ NATURAL PHENOMENA HAZARDS MITIGATION}

C-1 
HNF-SD-GN-ER-507, REV 0

This page intentionally left blank. 
HNF-SD-GN-ER-507 REV 0

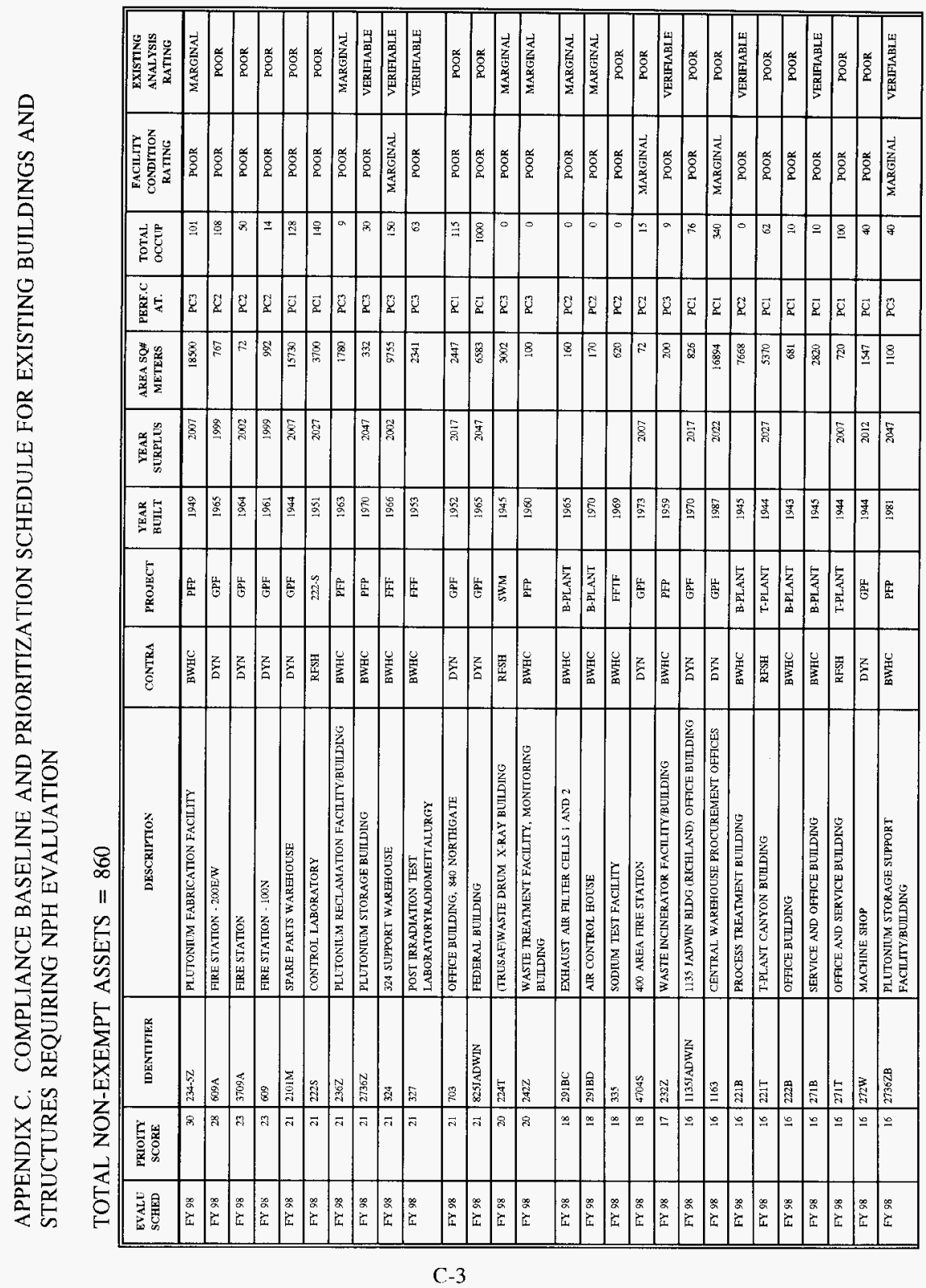




\begin{tabular}{|c|c|c|c|c|c|c|c|c|c|c|c|c|c|c|c|c|c|c|c|c|c|c|c|c|c|c|c|c|c|}
\hline 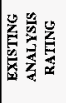 & 咅 & 岁 & 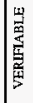 & 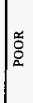 & 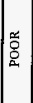 & 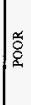 & 哭 & 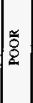 & 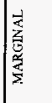 & 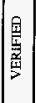 & 然 & 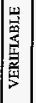 & 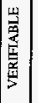 & 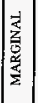 & 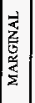 & 总 & 営 & 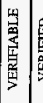 & 恶 & 莫 & $\begin{array}{ll}\approx \\
\varnothing\end{array}$ & 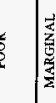 & 党 & 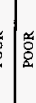 & 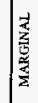 & 唄 & 徎 & 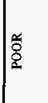 & 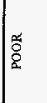 \\
\hline 至象 & 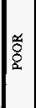 & 苂 & 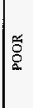 & : & 然 & 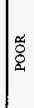 & 蒿 & 产 & 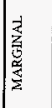 & 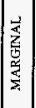 & 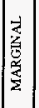 & 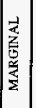 & 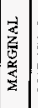 & $\mid \begin{array}{l}7 \\
\frac{7}{2} \\
\frac{2}{0} \\
\frac{2}{2} \\
\frac{2}{2}\end{array}$ & 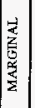 & $\begin{array}{l}3 \\
\frac{3}{3} \\
\frac{2}{2} \\
\frac{2}{2} \\
\end{array}$ & 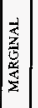 & 亭 & 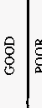 & & \% & శ్ & : & 2. & 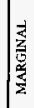 & 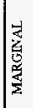 & 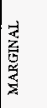 & 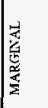 & $\begin{array}{l}\vec{z} \\
\frac{z}{2} \\
\frac{2}{2} \\
\frac{2}{2}\end{array}$ \\
\hline 离害 & 8 & 0 & 0 & 2 & 8 & 8 & $\Rightarrow$ & $\Rightarrow$ & 0. & \pm & $\therefore$ & $\Rightarrow$ & 8 & 0 & 0 & $\Rightarrow$ & 0 & 2 & 8 & \begin{tabular}{l|l}
$\infty$ & $\infty$
\end{tabular} & \begin{tabular}{l|l}
$\infty$ & $a$
\end{tabular} & 은 & 9 in & $\begin{array}{l}0 \\
\end{array}$ & $m$ & st & $\because$ & $\approx$ & : \\
\hline 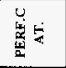 & $\vec{\Sigma}$ & $\tilde{D}^{\circ}$ & $\tilde{E}$ & $\bar{\Xi}$ & $\bar{g}$ & $\bar{\Omega}$ & $\bar{\Xi}$ & $\overline{\mathrm{C}}$ & 0 & $\tilde{\Sigma}$ & 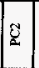 & 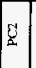 & $\Xi$ & $\Xi$ & 0 & $\Xi$ & 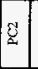 & 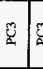 & $\Xi$ & $\bar{\Sigma}$ & $\overrightarrow{\mathrm{Q}} \overrightarrow{\mathrm{g}}$ & $\vec{g}$ & $\overrightarrow{\mathrm{g}}$ & $\vec{J}$ & $g$ & $\bar{\Sigma}$ & $\bar{Q}$ & $\bar{\Sigma}$ & $\bar{\varepsilon}$ \\
\hline 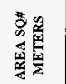 & 웟 & $\vec{m}$ & 票 & $\stackrel{9}{\Xi}$ & 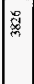 & 总 & 5 & 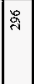 & $\stackrel{\circ}{\pi}$ & $E$ & 等 & 密 & \begin{tabular}{|l|l|}
$g$ \\
g
\end{tabular} & $\because$ & 至 & 5 & 落 & 京 & 8 & 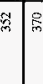 & \begin{tabular}{l|l}
$R$ & R \\
\end{tabular} & 桑 & \begin{tabular}{l|l} 
\\
$g$
\end{tabular} & 5 & 會 & $\frac{\$}{5}$ & $\frac{8}{8}$ & 桑 & 8 \\
\hline 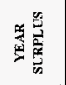 & 둘 & & & & & 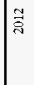 & & 兒 & & 氛 & & & & & & & & 今్ & 害 & \begin{tabular}{l|l} 
\\
$\bar{z}$
\end{tabular} & 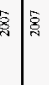 & 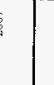 & 究 & s: & & 8 & 멸 & 韧 & वै \\
\hline 管 & \# & 兽 & g & 青 & 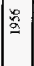 & 2 & $\overline{2}$ & 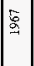 & 愛 & 点 & $\underset{2}{2}$ & 畄 & \begin{tabular}{l|l}
$\infty$ \\
$\vdots$ \\
$\Xi$
\end{tabular} & \begin{tabular}{|l|l}
2 \\
2
\end{tabular} & 䚄 & $\mathscr{E}$ & $\begin{array}{l}\infty \\
\underline{\infty} \\
\underline{g}\end{array}$ & $\begin{array}{l}2 \\
g\end{array}$ & $\begin{array}{l}0 \\
0\end{array}$ & \begin{tabular}{l|l}
$\mathrm{E}$ & $\mathrm{Z}$ \\
\end{tabular} & $\begin{array}{l} \\
\end{array}$ & 8 & \begin{tabular}{l|l}
8 & $\vec{g}$ \\
\end{tabular} & $\mathrm{~g}$ & 骂 & 桴 & 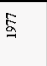 & 空 & 点 \\
\hline 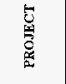 & 竞 & 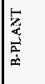 & 蓖 & 莸 & 范 & 岕 & 岕 & 䓌 & 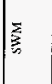 & 勇 & 崖 & 挋 & 在 & 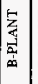 & 﨎 & 妾 & 崖 & \begin{tabular}{l|l}
$\frac{1}{2}$ \\
$\frac{1}{2}$
\end{tabular} & 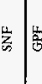 & 莸 㞾 & 岕 怠 & 竧 & 兵 & 焉 & 宏 & 岕 & 忘 & 营 & 岕 \\
\hline $\overrightarrow{\underline{z}}$ & 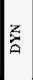 & 产 & 营 & 晃 & Z & 曾 & 兑 & 音 & 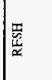 & $\mid \begin{array}{l}\text { 密 } \\
\text { 离 }\end{array}$ & 崖 & 密 & 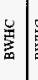 & 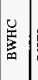 & 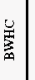 & 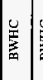 & 产 & 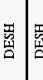 & 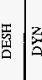 & z & z: & 崖 & 跣 & 点 & 宦 & 放 & Z्र & 晉 & 党 \\
\hline 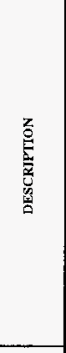 & 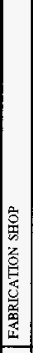 & 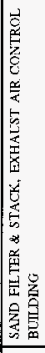 & 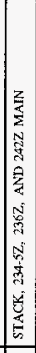 & 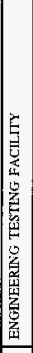 & 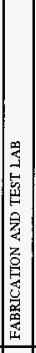 & 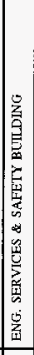 & 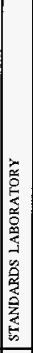 & 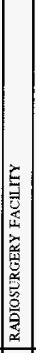 & 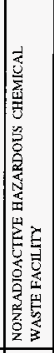 & 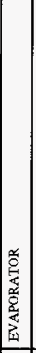 & 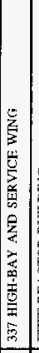 & 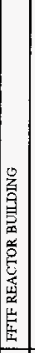 & 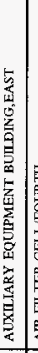 & 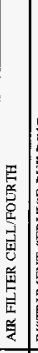 & 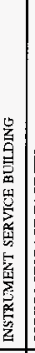 & 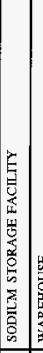 & 里 & 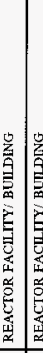 & 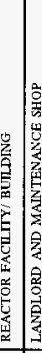 & 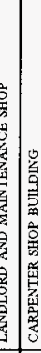 & 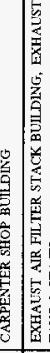 & 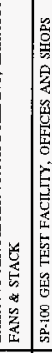 & 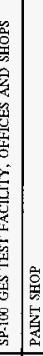 & 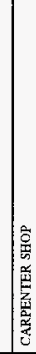 & 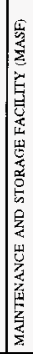 & 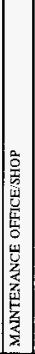 & 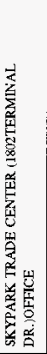 & 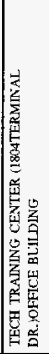 & 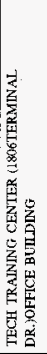 \\
\hline 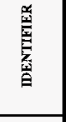 & ह & 䇾 & $\bar{s}$ & 啹 & 总 & 沓 & $\frac{a}{E}$ & $\frac{90}{2}$ & $\frac{0}{0}$ & $\begin{array}{l}\widehat{\int} \\
\text { gुd } \\
\end{array}$ & \begin{tabular}{|l}
0 \\
0 \\
0
\end{tabular} & 8 & 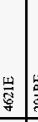 & 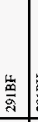 & 䟧 & 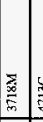 & 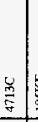 & 虽 & 童 & 恶 & 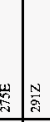 & 离 & 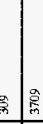 & $\sum_{m}^{m}$ & 8 & $\exists$ & 总 & 箘 & 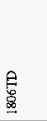 \\
\hline 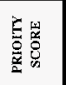 & 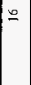 & $\simeq$ & 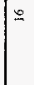 & 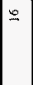 & 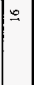 & 5 & 10 & $\%$ & 9 & \pm & $\exists$ & \pm & $\begin{array}{ll} \pm & \end{array}$ & 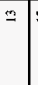 & $m$ & 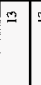 & $=$ & $\therefore=$ & $\therefore 1$ & 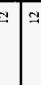 & 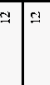 & & 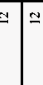 & \pm & I & $\Rightarrow$ & $\exists$ & $=$ & $=$ \\
\hline 胥言 & $\stackrel{2}{\circ}$ & $\stackrel{\infty}{x}$ & ${ }_{\infty}^{\infty}$ & 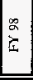 & 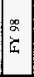 & $\stackrel{x}{x}$ & 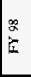 & 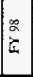 & 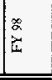 & $\omega_{i}^{\infty}$ & 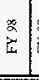 & $\begin{array}{l}\infty \\
\vdots \\
4 \\
4 \\
4\end{array}$ & 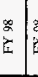 & 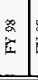 & $\stackrel{8}{\sim}$ & 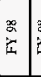 & 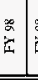 & \begin{tabular}{l|l|l}
$\infty$ & $\infty$ \\
$\vdots$ & \multicolumn{1}{c}{} \\
\end{tabular} & 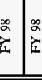 & $\frac{8}{2}$ & 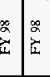 & 要 & \begin{tabular}{l|l}
$\infty$ \\
$\vdots$ \\
$z$
\end{tabular} & $g$ & ż & $\mid \begin{array}{l}m \\
z \\
r\end{array}$ & 㫧 & $\begin{array}{l}\% \\
2 \\
z\end{array}$ & 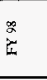 \\
\hline
\end{tabular}




\begin{tabular}{|c|c|c|c|c|c|c|c|c|c|c|c|c|c|c|c|c|c|c|c|c|c|c|c|c|c|c|c|c|c|c|c|}
\hline 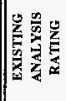 & 哭 & 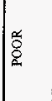 & 总 & 总 & 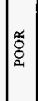 & 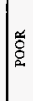 & $\stackrel{9}{\circ}$ & 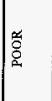 & 产 & 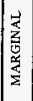 & 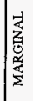 & 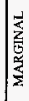 & $\mid \begin{array}{l}3 \\
2 \\
0 \\
0 \\
\frac{2}{2} \\
2\end{array}$ & 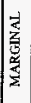 & 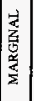 & 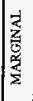 & 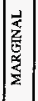 & $\begin{array}{l}\vec{z} \\
\frac{1}{5} \\
\frac{2}{5} \\
\frac{\alpha}{2} \\
\frac{2}{2}\end{array}$ & 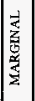 & 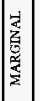 & 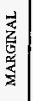 & $\mid \begin{array}{l}\frac{1}{2} \\
\frac{z}{c} \\
\frac{\alpha}{2} \\
\frac{\alpha}{2}\end{array}$ & 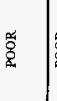 & 苂 & $\mid$ & 然 & 崫 & 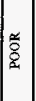 & 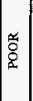 & 范 & 3 \\
\hline 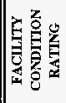 & 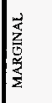 & 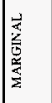 & 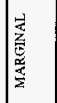 & 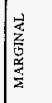 & 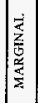 & 竞 & 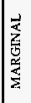 & 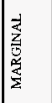 & 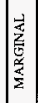 & 产 & हे & हे & 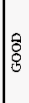 & 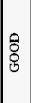 & 总 & ठ̊. & 离 & 宮 & 产 & 产 & 总 & 表 & 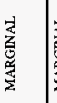 & 言 & 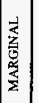 & \begin{tabular}{|l|} 
\\
$\frac{\pi}{2}$ \\
$\frac{2}{2}$ \\
$\frac{2}{2}$ \\
$\frac{2}{2}$
\end{tabular} & 这 & 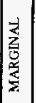 & $\begin{array}{l}3 \\
\frac{3}{2} \\
\frac{2}{2} \\
\frac{\alpha}{2} \\
\end{array}$ & & 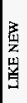 \\
\hline 武客 & : & $\%$ & $\because 8$ & $\frac{1}{10}$ & $\approx$ & 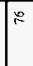 & $\div$ & $\approx$ & & 0 & $\sigma$ & \% & 0 & 0 & 0 & 0 & 0 & 0 & 0 & 0 & 0 & 0 : & 8 & \begin{tabular}{|l|} 
\\
\end{tabular} & $\therefore$ & $\infty$ & 8 & 8 & is & $P$ & $\therefore$ \\
\hline 离 & $\bar{\Xi}$ & $\bar{L}$ & $\bar{E}$ & $\bar{\Xi}$ & 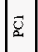 & $\overrightarrow{\mathrm{Q}}$ & $\bar{E}$ & $\bar{\Sigma}$ & 岛 & 8 & 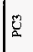 & 0 & E & 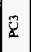 & 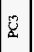 & 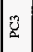 & 8 & 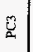 & 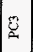 & 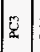 & 3 & 9 & $\overrightarrow{\mathrm{Q}}$ & $\overrightarrow{\mathrm{Q}}$ & $\overrightarrow{\underline{Q}}$ & $\overline{\mathrm{Q}}$ & $\bar{\varepsilon}$ & $\ddot{\mathrm{L}}$ & $\overrightarrow{\tilde{\omega}}$ & 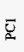 & $\xi$ \\
\hline 跣 & $\frac{8}{9}$ & 㝵 & $\frac{8}{8}$ & $\frac{8}{7}$ & 覀 & 总 & 票 & 영 & $\hat{\mathrm{s}}$ & 랄 & 善 & 总 & $\ddot{m}$ & $\ddot{e}$ & $\mathbb{E}$ & 崫 & 曽 & d & $\mathrm{F}$ & : & E & 窟 & 8 & 高 & 敢 & 8 & $E$ & है & gे & 영 & 8 \\
\hline$\approx$ & है & (5) & $\frac{1}{2}$ & בे & 苛 & 今. & 휴 & 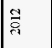 & ث⿳亠口冋口 & $\frac{8}{8}$ & 옥 & 迹 & 옥 & 产 & 음 & 产 & 总 & gू & 윰 & है & 올 & 总 & 总 & 商 & 愛 & 商 & & & 产 & 경 & $\overrightarrow{\widetilde{d}}$ \\
\hline 愬 & 喜 & 㕝 & 5 & $\Xi$ & 哥 & E & 唇 & 彦 & 鸹 & 哭 & 䠖 & 音 & 2 & 2 & 2 & 2 & 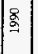 & \% & 8 & ळ & 8 & 空 & ğ & 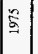 & 5 & $F$ & $\frac{2}{5}$ & 离 & $\overrightarrow{\mathrm{w}}$ & 票 & g \\
\hline 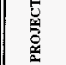 & 宸 & 峞 & $\frac{\Delta}{\hat{s}}$ & 崖 & 庇 & 崖 & 訔 & 訔 & 㗭 & $\sqrt{3}$ & 言 & $\overline{3}$ & $\bar{z}$ & $\bar{F}$ & $\bar{z}$ & $\bar{E}$ & 政 & $\overline{\tilde{z}}$ & $\bar{E}$ & $\sum$ & $\bar{z}$ & 辯蔼 & 忌 & 嵌 & 荘 & 岑 & i & te & 岕 & 焉 & 峛 \\
\hline$\widehat{\underline{z}}$ & za & z & za & z & $z$ & 公 & z & z & 点 & 䍠 & 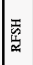 & 变 & 焉 & 范 & $\frac{1}{20}$ & 䍠 & 㘁 & 䁬 & 蔀 & 羂 & 蓉 & 舞 & 忘 & 店 & 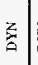 & $z$ & 离 & 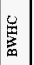 & 僁 & $\hat{a}$ & 蕟 \\
\hline 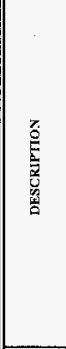 & 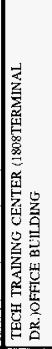 & 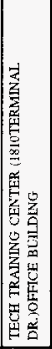 & 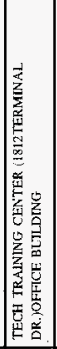 & 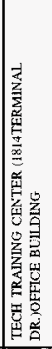 & 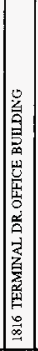 & 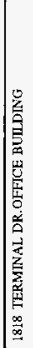 & 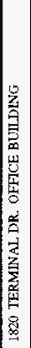 & 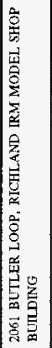 & 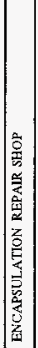 & 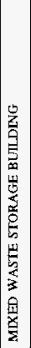 & 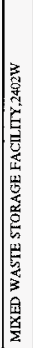 & 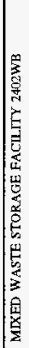 & 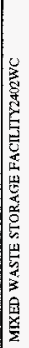 & 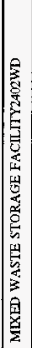 & 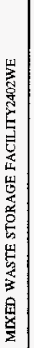 & 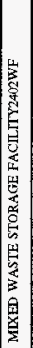 & 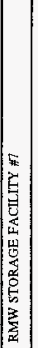 & 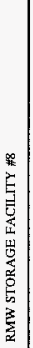 & 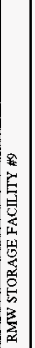 & 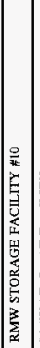 & 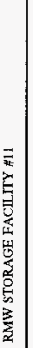 & 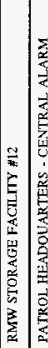 & d & 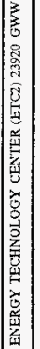 & 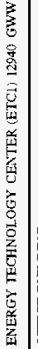 & 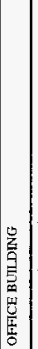 & 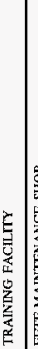 & 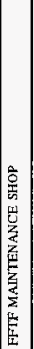 & 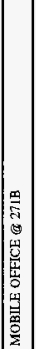 & 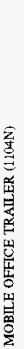 & 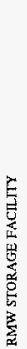 \\
\hline 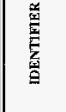 & 登 & 量 & 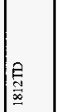 & \begin{tabular}{|l} 
P \\
紊 \\
\end{tabular} & 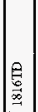 & 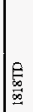 & 展 & 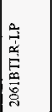 & 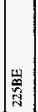 & 辛 & 量 & 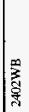 & 套 & 量 & 章 & 嵼 & 密 & 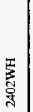 & 竭 & 点 & 总 & 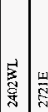 & $\underset{\vec{~}}{\vec{~}}$ & 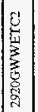 & 尊 & 8 & 等 & 骨 & $\frac{8}{2}$ & $\overline{8}$ & 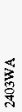 \\
\hline 등 & $=$ & $\Rightarrow$ & $=$ & $\Rightarrow$ & $\Rightarrow$ & $\exists$ & $\exists$ & $\Rightarrow$ & $\Rightarrow$ & $=$ & $\Rightarrow$ & $=$ & $=$ & $=$ & $=$ & $=$ & $=$ & $=$ & $=$ & $\Rightarrow$ & $=$ & $\Rightarrow=$ & $=$ & $=$ & $\Rightarrow$ & $\Rightarrow$ & $\Rightarrow$ & $=$ & $=$ & & $\bar{\rho}$ \\
\hline 富害 & \begin{tabular}{l}
$\infty$ \\
\hdashline \\
$x$
\end{tabular} & $\begin{array}{l}\% \\
\frac{2}{2} \\
:\end{array}$ & 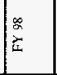 & 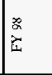 & $\left|\begin{array}{l}\% \\
z \\
z \\
z\end{array}\right|$ & 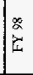 & $\stackrel{\circ}{\approx}$ & $\begin{array}{l}\infty \\
\% \\
\tilde{L}\end{array}$ & $\mid \begin{array}{l}\infty \\
\vdots \\
i \\
i\end{array}$ & $\stackrel{\infty}{\circ}$ & ※ & 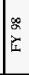 & $\overbrace{>}^{2}$ & 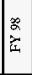 & 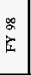 & $\approx$ & 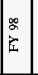 & 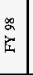 & 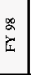 & $\begin{array}{l}\infty \\
x \\
z \\
z\end{array}$ & 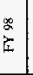 & $\begin{array}{ll}* \\
0 \\
0\end{array}$ & 象 & 象 & 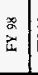 & \begin{tabular}{l}
$\infty$ \\
\multirow{L}{*}{} \\
$\tilde{L}$
\end{tabular} & 2 & 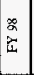 & 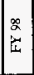 & & 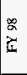 \\
\hline
\end{tabular}


HNF-SD-GN-ER-507 REV 0

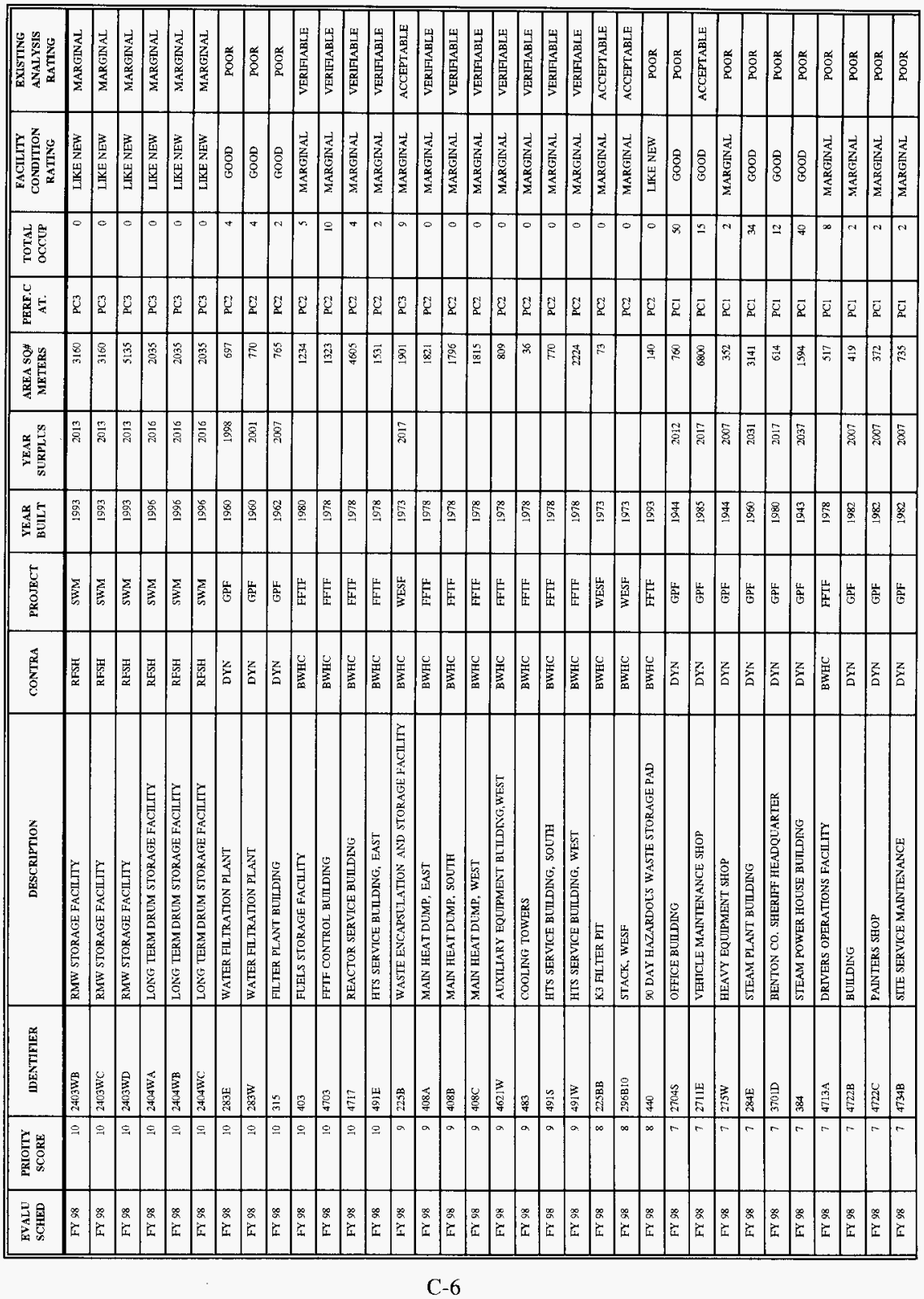




\begin{tabular}{|c|c|c|c|c|c|c|c|c|c|c|c|c|}
\hline $\begin{array}{l}\text { EVALU } \\
\text { SCHED }\end{array}$ & $\begin{array}{l}\text { PRIOITY } \\
\text { SCORE }\end{array}$ & IDENTIFIER & DESCRIYTION & CONTRA & PROJECT & $\begin{array}{l}\text { YEAR } \\
\text { BLILT }\end{array}$ & $\begin{array}{l}\text { YEAR } \\
\text { SURPLLS }\end{array}$ & $\begin{array}{l}\text { AREA } S Q / 4 \\
\text { METERS }\end{array}$ & $\begin{array}{c}\text { PERF.C } \\
\text { AT. }\end{array}$ & $\begin{array}{l}\text { TOTAL } \\
\text { OCCUP }\end{array}$ & $\begin{array}{l}\text { FACILITY } \\
\text { CONDITION } \\
\text { RATING }\end{array}$ & $\begin{array}{l}\text { EXISTING } \\
\text { ANALYSIS } \\
\text { RATING }\end{array}$ \\
\hline FY 98 & 7 & 4862 & $\begin{array}{l}\text { FMEF ENTRY WINO OFFCE FMEF OFFICE - ENTRY } \\
\text { WLNG }\end{array}$ & BWHC & FFF & 1984 & 2037 & 3721 & PC1 & 30 & GOOD & MARGINAL \\
\hline $\mathrm{FY} @ 8$ & $?$ & $662 \mathrm{~A}$ & PATROL EXERCISE \& TRAINING FACILITY \& PTA & DYN & GPF & 1983 & 2017 & 465 & PC1 & 5 & MARGNAL & POOR \\
\hline FY 98 & 7 & MO273 & MOBLE OFFICE (G) PUREX (OUTSIDE FENCE) & DYN & GPF & 1990 & 2007 & 859 & PCl & 81 & GOOD & POOR \\
\hline FY 98 & 6 & 6270 & ENVIRONMENTAL DATA REMEDIALTRACKING & RFSH & HAS & 1993 & 2024 & 435 & PC1 & 14 & LIKE NEW & MARGINAL \\
\hline FY & 6 & MO294 & MOBILE OFFICE & DYN & GPF & 1995 & 2012 & 1374 & PC1 & 90 & LIKE NEW & POOR \\
\hline FY 98 & 6 & MOs70 & MOBLLE OFFICE & DYN & GPF & 1993 & 2067 & 312 & $\mathrm{PCl}$ & 26 & LIKE NEW & POOR \\
\hline FY 98 & 6 & M0971 & MOBLE OFFICE & DYN & GPF & 1993 & 2007 & $31 ?$ & $\mathrm{PCl}$ & 26 & LIKE NEW & POOR \\
\hline $\mathrm{FY} 98$ & 0 & $1330 \mathrm{~N}$ & 100-N WASTE STORAGE FACILTTY & PHMC & & & & & $\mathrm{PCy}$ & & & \\
\hline FY 98 & 0 & $1777 \mathrm{TD}$ & 1777 TERMINAL DRNE, RICHLAND & NUMTC & & & & & PCy & & & \\
\hline $5 Y 98$ & 0 & MO481 & OPERATTONS OFFICE TRAILER (AKA 103LW) & RFSH & & & & 446 & $\mathrm{PCY}$ & & & \\
\hline FY 98 & & $1100 \mathrm{~A} A \mathrm{DWIN}$ & $\begin{array}{l}1100 \text { JADWNN BLDG (RICHLAND)HEADQUARTERS } \\
\text { OFFICE BUWDING }\end{array}$ & DYN & & & & 4423 & $\mathrm{PCY}$ & & & \\
\hline$F Y 98$ & & 1112 & FLEL STORAGE BUTI DIYGG & PHMC & & & & 6 & $\mathrm{PCx}$ & & & \\
\hline$F Y 98$ & & $1112 \mathrm{NA}$ & MICROWAVE TOWER ANNEX & PHMC & & & & 13 & $\mathrm{PCx}$ & & & \\
\hline FY 98 & & 11201 & TOOL SHED & PHMC & & & & & PCX & & & \\
\hline FY 98 & & $1171 \mathrm{~A}$ & BUS WASH FACILTY & PHMC & & & & 24 & $\mathrm{PCx}$ & & & \\
\hline FY 98 & & $1171 \mathrm{C}$ & SPARE PARTS STORAGE SHED & PHMC & & & & 93 & $\mathrm{PCx}$ & & & \\
\hline FY 98 & & 1178 & CHOLORING STORAGE STRUCTURE & PHмC & & & & & $\mathrm{PCx}$ & & & \\
\hline$F Y 98$ & & 1180 & PESTICIDE STORAGE BUILDING & PHMC & & & & 6 & $\mathrm{PCx}$ & & & \\
\hline FY 98 & & 1200JADWIN & $\begin{array}{l}\text { TCPC (1200 JADWIN, RICHLAND) TCPC OFEICE } \\
\text { BUIDING }\end{array}$ & DYN & & & & 8616 & $\mathrm{PCy}$ & & & \\
\hline FY 98 & & $1515 \mathrm{~N}$ & MULTICRAFT SHOP & PHMC & & & & 446 & $\mathrm{PCX}$ & & & \\
\hline FY 98 & & $1516 \mathrm{~N}$ & CARPENIER SHOP & PHMC & & & & 45 & $\mathbf{P C x}$ & & & \\
\hline FY 98 & & $1517 \mathrm{~N}$ & PAINT SHOP & PHMC & & & & 74 & $\mathrm{PCx}$ & & & \\
\hline FY 98 & & $1518 \mathrm{~N}$ & FIXED METAL ELECTRICAL SHOP & PHMC & & & & 74 & $\mathrm{PCx}$ & & & \\
\hline FY 98 & & $1519 \mathrm{~N}$ & FIXED METAL FTTTERS SHOP & PHMC & & & & 74 & $\mathrm{PCx}$ & & & \\
\hline FY $\$ 8$ & & $151 \mathrm{~B}$ & PRIMARY SUBSTATION & PHMC & & & & 610 & $P C x$ & & & \\
\hline FY 98 & & 1510 & PRIMARY SLBSTATION & PHMC & & & & 550 & PCX & & & \\
\hline FY 98 & & $1614 \mathrm{~K} 3$ & ENVTRONMENTAL MONTORING STATION & PHMC & & & & 0 & $\mathbf{P C x}$ & & & \\
\hline FY 98 & & 1701KA & GUARD OFFLCE & PHMC & & & & & $\mathrm{PCx}$ & & & \\
\hline FY 98 & & 1717AKE & FAN HOUSE BY 1717 & PHMC & & & & & $\mathrm{PC} x$ & & & \\
\hline FY 98 & & 1717KE & MAINTENANCE TRANSPORTATION SHOP & FHMC & $100 \mathrm{~K}$ & & & & $\mathrm{PCx}$ & & & \\
\hline FY 98 & & $1724 \mathrm{~N}$ & $\begin{array}{l}\text { NITROGEN ELECTRICAI. CONTROL BLDG. } \\
\text { PROPOSED }\end{array}$ & PHMC & & & & & $\mathrm{PCX}$ & & & \\
\hline FY 98 & & $183.6 \mathrm{KW}$ & LIME FEEDER BUILDINGLIME FEEDER BUILDNG & PHMC & & & & 86 & PCx & & & \\
\hline
\end{tabular}




\begin{tabular}{|c|c|c|c|c|c|c|c|c|c|c|c|c|}
\hline $\begin{array}{l}\text { EVALU } \\
\text { SCHED }\end{array}$ & $\begin{array}{l}\text { PRIOITY } \\
\text { SCORE }\end{array}$ & IDENTIFIER & DESCRIPTION & CONTRA & PROIECT & $\begin{array}{l}\text { YEAR } \\
\text { BUIET }\end{array}$ & $\begin{array}{c}\text { YEAR } \\
\text { SURPLUS }\end{array}$ & $\begin{array}{l}\text { AREA SQH } \\
\text { METERS }\end{array}$ & $\begin{array}{l}\text { PERF.C } \\
\text { AT. }\end{array}$ & $\begin{array}{l}\text { TOTAL } \\
\text { OCCCPP }\end{array}$ & $\begin{array}{l}\text { FACILITY } \\
\text { CONDITION } \\
\text { RATING }\end{array}$ & $\begin{array}{l}\text { EXISTING } \\
\text { AYALYSIS } \\
\text { RATING }\end{array}$ \\
\hline $\mathrm{FY} 98$ & & $1835 \mathrm{TD}$ & $\begin{array}{l}\text { VITRO BLIDG (1835 TERMINAL } \\
\text { DR.,RICHLANDIVITRO BUILDING }\end{array}$ & MLLT & & & & & $\mathrm{PCy}$ & & & \\
\hline FY 98 & & $183 \mathrm{D}$ & $\begin{array}{l}\text { FILTER PLANT POWER OPERATION } \\
\text { FACILITYFILTER PLANT BLILDING }\end{array}$ & PHMC & & & & 6055 & $\mathrm{PCx}$ & & & \\
\hline FY 98 & & $183 \mathrm{KE}$ & $\begin{array}{l}\text { FILTER PLANT HEAD HOLSE, CHLORINEWHCHEAD } \\
\text { HOISE,CHI.ORLNE }\end{array}$ & PHMC & & & & 1741 & $\mathrm{PCx}$ & & & \\
\hline FY 98 & & 1920HLSBRO & 1920 E. HLLSBORO (CENEX WARHOLSE) & PHMC & & & & 743 & $\mathrm{PCx}$ & & & \\
\hline FY 98 & & 1981SNYDER & $\begin{array}{l}\text { 1981 SNYDER, SUITE 1, RICHLANDOFFICE } \\
\text { BUWWLG }\end{array}$ & DYN & & & & & PCy & & & \\
\hline FY 98 & & $201 \mathrm{~W}$ & $\begin{array}{l}\text { INSTRLMENT BUILDING SE CRN ZWOWINSTRUMENI } \\
\text { BUTLDING }\end{array}$ & PHMC & & & & 36 & $\mathrm{PCx}$ & & & \\
\hline FY 98 & & $204 \mathrm{AR}$ & WASTE LNLOADLVG FACILITY/STATHON/BULUING & LMHC & & & & 238 & $\mathrm{PCx}$ & & & \\
\hline FY 98 & & $207 \mathrm{~T}$ & WATER REJENTION BASIN & LMHC: & T-PLANT & & & & $\mathrm{PCX}$ & & & \\
\hline FY 98 & & $209 \mathrm{~A}$ & $\begin{array}{l}\text { INSTRUMENT ALR COMPRESSOR BUILING } \\
\text { (2-84)OFFICE BUILDNG }\end{array}$ & LMHC & & & & 8 & PCx & & & \\
\hline FY 98 & & $209 \mathrm{E}$ & ENVIRONMENTAL WASTE OPERATIONS & LMHC & & & & 742 & $\mathrm{PCx}$ & & & \\
\hline FY 98 & & $209 \mathrm{EA}$ & 9. DAY STORAGE PAD & LMHC: & & & & & $\mathrm{PCx}$ & & & \\
\hline FY 98 & & 2 LOHV & MWTFHWVP CONSTRUCTION WAREHOUSE & PHMC & $222-\mathrm{s}$ & & & & $\mathrm{PCx}$ & & & \\
\hline FY 98 & & $210 \mathrm{~A}$ & $\begin{array}{l}\text { OLL DRUM STORAGE. PUREXOIL DRUM STORAGE } \\
\text { BULDING }\end{array}$ & PHMC & & & & 67 & $\mathrm{PCx}$ & & & \\
\hline FY 98 & & $213 \mathrm{~K}$ & MAGAZINE WASTE STORAGE BUILDING & PHMC & & & & 49 & $\mathrm{PCX}$ & & & \\
\hline FY 98 & & $213 \mathrm{~W}$ & WASTE COMPACTOR BLHLDLNG & LMHC & & & & 80 & $\mathrm{PCx}$ & & & \\
\hline FY 98 & & 2:3WTK1 & CATCH TANK & LMHC & & & & & $\mathrm{PCx}$ & & & \\
\hline FY 98 & & $214 \mathbf{B}$ & HANFORD WASTE VITRIFICATIONPLANT & PHMC & PUREX & & & & $P C x$ & & & \\
\hline FY 98 & & $215 \mathrm{~A}$ & VALVE CONTROL FACILITY, CRUBS WELLS ECT & EMHC & & & & 9 & $\mathbf{P C x}$ & & & \\
\hline FY 98 & & $216 \mathrm{~A} 271$ & VALVE CONTROL HOLSE & LMHC & & & & 9 & $\mathrm{PCx}$ & & & \\
\hline FY 98 & & $216 \mathrm{~A} 30$ & CRIB A-30 & LMHC & PLREX & & & & $\mathrm{PCx}$ & & & \\
\hline FY 98 & & $216 \mathrm{~A} 301$ & DIVERSION BOX & LMHC & & & & & $\mathrm{PCx}$ & & & \\
\hline FY 98 & & $216 \mathrm{~A} 302$ & DNVERSION BOX & LMHC & & & & & $\mathrm{PCx}$ & & & \\
\hline FY 98 & & $216 \mathrm{~A} 372$ & CRIB A37-2 & LMHC & & & & & $P C x$ & & & \\
\hline FY 98 & & $216 \mathrm{~A} 40$ & $\begin{array}{l}\text { PUREX PROCESS CONDENSATE REPLACEMENT } \\
\text { CRIB }\end{array}$ & LMEC & & & & & PCx & & & \\
\hline FY 98 & & $215 \AA 45$ & CRIB A45 & LMHC & PUREX & & & & $\mathrm{PCx}$ & & & \\
\hline $\mathrm{FY} 98$ & & $215 A 908$ & CRIB CONTROL STRUCTURE & LMHC & & & & & $\mathrm{PCx}$ & & & \\
\hline FY 98 & & $216 \mathrm{~A} 8$ & CRIB A8 & I.MHC & A-FARM & & & & $\mathbf{P C x}$ & & & \\
\hline FY 98 & & $216 \mathbf{R} 23 \mathrm{~A}$ & DIVERSLN BOX ON 216B2 3 PIPELINE & LMHC & & & & & PCX & & & \\
\hline FY 98 & & $216 \mathbf{B} 3 \mathbf{A}$ & PONDS & LMHC & & & & & PCX & & & \\
\hline FY 98 & & $216 \mathrm{~B} 3 \mathrm{AI}$ & 2 216B3A (FRST LOBE) CONTROL STRUICTURE & LMHC & & & & & $\mathrm{PCX}$ & & & \\
\hline
\end{tabular}




\begin{tabular}{|c|c|c|c|c|c|c|c|c|c|c|c|c|}
\hline $\begin{array}{l}\text { EVALE } \\
\text { SCHED }\end{array}$ & $\begin{array}{l}\text { PRIOITY } \\
\text { SCORE }\end{array}$ & IDENTIFIER & DESCRIFTION & CONTRA & PROJECT & $\begin{array}{l}\text { YEAR } \\
\text { BULT T }\end{array}$ & $\begin{array}{l}\text { YEAR } \\
\text { SURPLIS }\end{array}$ & $\begin{array}{l}\text { AREA SQH } \\
\text { METERS }\end{array}$ & $\begin{array}{l}\text { PERF.C } \\
\text { AT. }\end{array}$ & $\begin{array}{l}\text { TOTAL } \\
\text { OCCLP }\end{array}$ & $\begin{array}{c}\text { FACILITY } \\
\text { CONDIFION } \\
\text { RATING }\end{array}$ & $\begin{array}{c}\text { EXISTING } \\
\text { ANALYSIS } \\
\text { RATING }\end{array}$ \\
\hline FY 98 & & $216 \mathrm{~B} 3 \mathrm{~B}$ & PONDS & EMHC & & & & & $\mathrm{PCx}$ & & & \\
\hline FY 98 & & $216 \mathrm{~B} 3 \mathrm{C}$ & PONDS & LMHC & & & & & $\mathrm{PCx}$ & & & \\
\hline FY 98 & & $216 \mathbf{B} 55$ & CRIB B55 RETENTION & LMHC & & & & & $\overline{\mathrm{PCx}}$ & & & \\
\hline FY $\$ 8$ & & $216 \mathrm{~B} 62$ & CRIB B62 RETENTTON & LMHC & & & & & $\mathrm{PCx}$ & & & \\
\hline FY 98 & & $216 \mathrm{~B} 63$ & RETENTION DITCH B 63 & LMHC & & & & & $\mathrm{PCX}$ & & & \\
\hline FY 98 & & $2.16 e+3 !$ & $\begin{array}{l}\text { 200 EAST AREA CONTINGENCY POLT RETENTTON } \\
\text { BASN }\end{array}$ & LMHC & & & & & $\mathrm{PCX}$ & & & \\
\hline FY 98 & & $216 \mathrm{E} 28 \mathrm{~A}$ & CONTRNGENCY POND CONTROLSTRUCTURE & LMHC & & & & & PCK & & & \\
\hline FY 98 & & $216 \mathrm{E} 28 \mathrm{~B}$ & $\begin{array}{l}\text { CONTINGENCY POND BYPASS CONTROL } \\
\text { STRUCTURE }\end{array}$ & LMHC & & & & & $\mathrm{PCX}$ & & & \\
\hline FY98 & & $216 \$ 25$ & CRIB S25 & LMHC & S-FARM & & & & PCx & & & \\
\hline FY 98 & & 216526 & CRUB \$26 & LMHC & S-FARM & & & & $\mathrm{PCK}$ & & & \\
\hline FY 98 & & $216 \mathrm{T1}$ & TRENCHES DICH DITCH Tl & LMHC & & & & & $\mathrm{PCx}$ & & & \\
\hline FY98 & & $216 \mathrm{~T} 42$ & DITCH & LMHC & & & & & $P C x$ & & & \\
\hline FY 98 & & $216 \mathrm{TY201}$ & TANKFLUSH & LMHC & & & & & $\mathrm{PCx}$ & & & \\
\hline FY 98 & & $216\lfloor 14$ & DTTCH L14/CATCH TANK & LMHC & \multicolumn{2}{|c|}{ U03/U-FARM } & & & PGx & & & \\
\hline$F Y 98$ & & $216 \mathrm{U16}$ & CRIB & LMHC & & & & & $\mathrm{PCx}$ & & & \\
\hline FY 98 & & $216 \mathrm{LI} 17$ & $\begin{array}{l}\text { REPLACEMENT CRIB VADOSE ZONE MONITORING } \\
\text { WELLS }\end{array}$ & LMHC & 103 & & & & $\mathrm{PCx}$ & & & \\
\hline FY 98 & & $216 \mathrm{WLC}$ & EFFLUENT CRIB & LMHC & & & & & $\mathrm{PCx}$ & & & \\
\hline FY 98 & & $216 \mathrm{Z20}$ & CRIB 720 & LMHC & & & & & $\mathrm{PCx}$ & & & \\
\hline FY 98 & & $216: 21$ & BASDNS POND Z21 & LMHC & & & & & $\mathrm{PCX}$ & & & \\
\hline FY 98 & & $218 \mathrm{~B}$ & EMERGENCY FQUTPMENT STORAGE SHED & PHMC & B-PLANT & & & 2 & $P C x$ & & & \\
\hline FY 98 & & $218 \mathrm{E} 16101$ & GROUT DISPOSAL VAULT \#1 & LMHC & & & & & $\mathrm{PCx}$ & & & \\
\hline FY 98 & & $218 W 5-252$ & BUILDING ELECTRICAL CONTROL & PHMC & & & & & $\mathrm{PCX}$ & & & \\
\hline FY 98 & & 218W5-252A & BIMLDING ELECTRICAL, CONTROL & PHMC & & & & & $\mathrm{PCX}$ & & & \\
\hline FY 98 & & $2200 \mathrm{~B}$ & CONSTRUCTION PORTAL MONITORING BUILDING & PHMC & & & & 6 & $\mathbf{P C x}$ & & & \\
\hline FY 98 & & $2201 \mathrm{~B}$ & CONSTRUCTION ICE HOUSE & PHMC & & & & 27 & $\mathrm{PCx}$ & & & \\
\hline FY 98 & & $220 \mathrm{~A}$ & STEAM CONDENSATE SAMPLER BLDG & LMHC & & & & 8 & PCx & & & \\
\hline FY 98 & & $221 \mathrm{~A}$ & KEH PIPEFTTTER SHOP & PHMC & & & & 80 & $\mathrm{PCx}$ & & & \\
\hline FY 98 & & $222 S G$ & GAS BOTTLE STAGNG BUTLDNG & PHMC & $222-s$ & & & 41 & $\mathrm{PCx}$ & & & \\
\hline FY 98 & & $224 ! \mathrm{B}$ & LABORER'S STORAGE & PHMC & & & & & $\mathrm{PCx}$ & & & \\
\hline FY 98 & & $2242 \mathrm{~B}$ & CARPENTER SHOP & PHMC & & & & & $P C x$ & & & \\
\hline FY 98 & & $2244 \mathrm{~B}$ & MULTT CRAFT FABRICATION SHOP & PHMC & & & & 335 & $\mathrm{PCX}$ & & & \\
\hline FY 98 & & $2245 \mathrm{~B}$ & ELECTRICIAN'S LUNCHROOM/OFFICE & PHMC & & & & 74 & $\mathrm{PCx}$ & & & \\
\hline FY 98 & & $2247 \mathrm{~B}$ & IRONWORKER'S SHOP & PHMC & & & & 204 & $\mathrm{PCx}$ & & & \\
\hline
\end{tabular}




\begin{tabular}{|c|c|c|c|c|c|c|c|c|c|c|c|c|}
\hline $\begin{array}{l}\text { EVALU } \\
\text { SCHED }\end{array}$ & $\begin{array}{l}\text { PRIOITY } \\
\text { SCORE }\end{array}$ & IDENTIFIER & DESCRIPTION & CONTRA & PRO.JECT & $\begin{array}{l}\text { YEAK } \\
\text { BUILT }\end{array}$ & $\begin{array}{l}\text { YEAR } \\
\text { St'RPLUS }\end{array}$ & $\begin{array}{l}\text { AREA SQ" } \\
\text { METERS }\end{array}$ & $\begin{array}{l}\text { PERF.C } \\
\text { AT. }\end{array}$ & $\begin{array}{l}\text { TOTAL } \\
\text { OCCUP }\end{array}$ & $\begin{array}{l}\text { FACILITY } \\
\text { CONDITION } \\
\text { RATING }\end{array}$ & $\begin{array}{l}\text { EXISTING } \\
\text { ANALYSIS } \\
\text { RATING }\end{array}$ \\
\hline FY 98 & & $2249 \mathrm{~B}$ & BOTTLE STORAGE & PHMC & & & & & $\mathrm{PCx}$ & & & \\
\hline FY $\$ 8$ & & $225 \mathrm{EC}$ & LOCAL CONTROL UNIT S5C-13 & PHMC & & & & 11 & $\mathrm{PCx}$ & & & \\
\hline FY 98 & & $225 \mathrm{WA}$ & LOCAL CNTRL UNIT $55 \mathrm{C}-21$ & PHMC & & & & 11 & $\mathrm{PCx}$ & & & \\
\hline FY 98 & & $2309 \mathrm{~W}$ & SHEETMETAL SHOP & DYN & & & & 372 & PCy & & & \\
\hline FY 98 & & $2310 \mathrm{~W}$ & MATERIAL SIORAGE & PHMC & & & & 279 & $\mathrm{PCX}$ & & & \\
\hline FY 98 & & $2318 \mathrm{~W}$ & PAENTER SHOP & PHMC: & & & & & $\mathrm{PCx}$ & & & \\
\hline $\mathrm{FY} 98$ & & $231 \mathrm{~W} 15 !$ & VAULT & LMHC & & & & & $\mathrm{PCX}$ & & & \\
\hline FY $₫ 8$ & & 2400 E GROLT & DRY MATERIAL FACIITY CONTROL ROOM & LMHC & & & & & $\mathrm{PCx}$ & & & \\
\hline FY 98 & & 2402EA GROUT & DRY MATERIAL FACILITY UN'LOADING FACILITY & LMHC & & & & & $\mathrm{PCx}$ & & & \\
\hline FY 98 & & $2402 \mathrm{~EB}$ GROLT & DRY MATERLAL FACILTYY CEMENT SILO & LMHC & & & & & $P C x$ & & & \\
\hline FY98 & & 2402EC GROUT & DRY MATERLAL FACILITY FLY ASH SLLO & LMHC & & & & & $\mathrm{PCX}$ & & & \\
\hline FY98 & & 2402ED GROLT & DRY MATERIAL FACILITY POTTERY CLAY SUOO & LMHC & & & & & $\mathrm{PCX}$ & & & \\
\hline $\mathrm{FY} 98$ & & 2402EF GROUT & DRY MATERIAL FACILITY ATTAPULGITE CLAY & LMHC & & & & & $\mathrm{PCX}$ & & & \\
\hline FY 98 & & 2402EG GROUT & DRY MATERLAL FACILITY TRANSFER PUMP PII & LMHC & & & & & $\mathrm{PCx}$ & & & \\
\hline FY 98 & & $2402 \mathrm{WA}$ & MIXED WASTE STORAGE & PHMC & & & & & $\mathrm{PC} x$ & & & \\
\hline FY 98 & & $2403 E$ & $\begin{array}{l}\text { DMRHF DRY BLENDED STORAGE TRUCK } \\
\text { LOADOUT FACILITY }\end{array}$ & LMHC & & & & 19 & $\mathrm{PCx}$ & & & \\
\hline FY 98 & & $2403 \mathrm{EA}$ & COMPRESSOR LEAN-TO & LMHC & & & & 24 & $\mathrm{PCx}$ & & & \\
\hline FY 98 & & $2404 \mathrm{E}$ & COMPRESSOR/RAIL RECENVING & LMHC & & & & 21 & $\mathrm{PC} x$ & & & \\
\hline FY 98 & & $240 \mathrm{SI} 51$ & DIVERSION BOX & LMHC & S.FARM & & & & $\mathrm{PCX}$ & & & \\
\hline FY 98 & & $240 \$ 152$ & DIVERSTON BOX & LMHC & S-FARM & & & & $\mathrm{PCx}$ & & & \\
\hline $\mathrm{FY} 98$ & & $240 \$ 302 \mathrm{C}$ & ACD STORAGE TANK & LMHC & & & & & $\mathrm{PCx}$ & & & \\
\hline FY 98 & & $241 \mathrm{~A}$ & $\begin{array}{l}\text { WASTE DISPOSAL TANK FARM, } 6 \text { TANKSI MILLION } \\
\text { GAL EATANK FARMS } 6 \text { TANKS, } 2 \\
\text { DIVERSIONBOXESITANK FARM }\end{array}$ & LMHC & TKF & & & & $\mathrm{PCx}$ & & & \\
\hline FY 98 & & $241 \mathrm{~A} 01 \mathrm{~B}$ & Central Parto Pits, Shuice Pil & LMHC & A.Fam & & & & $\mathrm{PCX}$ & & & \\
\hline FY 98 & & $241 \mathrm{~A} 01 \mathrm{C}$ & Shice Pit & LMHC & A-Farn & & & & $\mathrm{PCx}$ & & & \\
\hline FY 98 & & 2A1A0ID & Shice Pit & LMHC & A-Farm & & & & $\mathrm{PCx}$ & & & \\
\hline FY 98 & & $241 \mathrm{AO1E}$ & Sluice Pit & LMHC & A-Farm & & & & $\mathrm{PCx}$ & & & \\
\hline FY 98 & & $241 \mathrm{AO} \mid \mathrm{H}$ & Distributcr Pil & LMHC & A-Farm & & & & $\mathrm{PCx}$ & & & \\
\hline FY 98 & & $241 \mathrm{~A} 02 \mathrm{~B}$ & Shluice Pit & LMHC & A-Farm & & & & $\mathrm{PCx}$ & & & \\
\hline FY 98 & & $241 \mathrm{~A} 02 \mathrm{C}$ & Shice Pit & LMHC & A-Fam & & & & $\mathrm{PCX}$ & & & \\
\hline FY 98 & & $241 \mathrm{~A} 02 \mathrm{D}$ & Shijee Pit & LMHC & A-Farm & & & & $\mathrm{PCX}$ & & & \\
\hline FY 98 & & $241 \mathrm{~A} 02 \mathrm{E}$ & Shuice Pit & LMAC & A Fam & & & & $\mathrm{PCX}$ & & & \\
\hline FY 98 & & $241 \mathrm{~A} 143$ & DFERSION BOXES & LMHC & & & & & $\mathrm{PCx}$ & & & \\
\hline FY 98 & & $241 \mathrm{~A} 14 \mathrm{4}$ & DNERSION BOXES & LMHC & & & & & $\mathrm{PCx}$ & & & \\
\hline
\end{tabular}




\begin{tabular}{|c|c|c|c|c|c|c|c|c|c|c|c|c|c|c|c|c|c|c|c|c|c|c|c|c|c|c|c|c|c|}
\hline 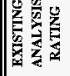 & & & & & & & & & & & & & & & & & & & & & & & & & & & & & \\
\hline 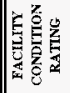 & & & & & & & & & & & & & & & & & & & & & & & & & & & & & \\
\hline | & & & & & & & & & & & & & & & & & & & & & & & & & & & & & \\
\hline 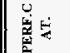 & 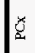 & 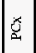 & $\mathscr{\Sigma}$ & 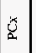 & 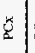 & 8 & 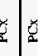 & 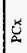 & 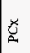 & 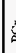 & 8 & 压 & 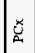 & $\check{\check{Z}}$ & $\check{\check{Z}}$ & $\frac{x}{2}$ & 818 & s. & $\frac{\gamma}{2}$ & 5 & 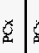 & 18 & 8 & $\breve{~}$ & $\underline{a}$ & 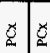 & 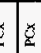 & & g \\
\hline 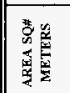 & & & & & & & & & 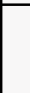 & & F & & & $\underline{\underline{z}}$ & & & $\infty$ & 8 & & & & & & & & & & & $\pi$ \\
\hline 㮍 & & & & & & & & & & & & & & & & & & & & & & & & & & & & & \\
\hline 盖畐 & & & & & & & & & & & & & & & & & & & & & & & & & & & & & \\
\hline 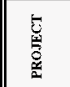 & & & & & & 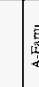 & 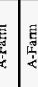 & 悬 & 浐 & 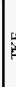 & & 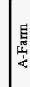 & 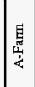 & | & 䨨 & & 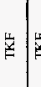 & c) & & 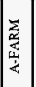 & & & & 舜 & 罜 & \begin{tabular}{l|l} 
\\
\end{tabular} & 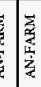 & & \\
\hline 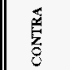 & 紊 & 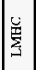 & $\frac{U}{\frac{U}{2}}$ & 峷 & 紊 & 摆 & 惫 & $\frac{y}{3}$ & $\frac{\underline{y}}{3}$ & 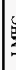 & 品 & 橧 & 量 & $\frac{y}{2}$ & 总 & 荺 & $\underline{z}$ & $\begin{array}{l}\frac{4}{2} \\
\frac{1}{3}\end{array}$ & $\underline{E}$ & $\frac{u}{\underline{x}}$ & 㩊 & $y$ & ty & 謩 & 慈 & $\frac{\mathrm{x}}{\mathrm{T}}$ & 兽 & & 㷼 \\
\hline 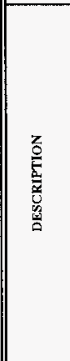 & 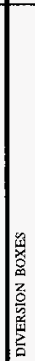 & 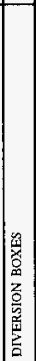 & 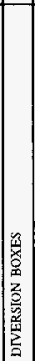 & 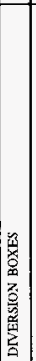 & 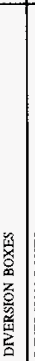 & 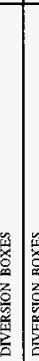 & 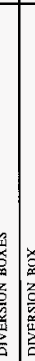 & 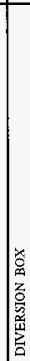 & 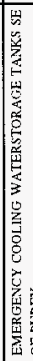 & & & 总 & 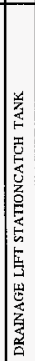 & 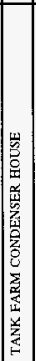 & 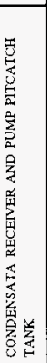 & 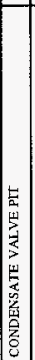 & 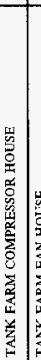 & 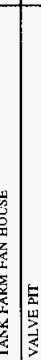 & $\mid \begin{array}{l}5 \\
\\
\end{array}$ & 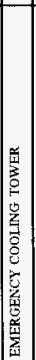 & 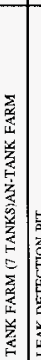 & 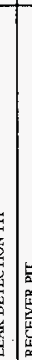 & 点 & 焉 & 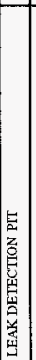 & 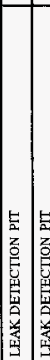 & 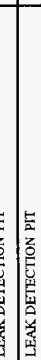 & 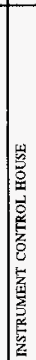 & 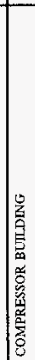 \\
\hline 总 & $\frac{3}{3}$ & 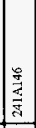 & \begin{tabular}{|l}
5 \\
$\frac{a}{3}$ \\
$\frac{a}{3}$ \\
\end{tabular} & 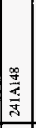 & \begin{tabular}{|l|l|} 
\\
\\
$\frac{3}{3}$ \\
\end{tabular} & 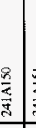 & 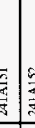 & $\frac{8}{8}$ & 高 & 3 & 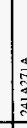 & 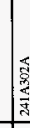 & \begin{tabular}{|l}
8 \\
$\frac{2}{2}$ \\
3 \\
\end{tabular} & \begin{tabular}{|l|}
$\vec{a}$ \\
\multirow{2}{*}{} \\
\end{tabular} & $\frac{3}{3}$ & \begin{tabular}{|l|} 
\\
$\frac{\bar{z}}{2}$ \\
$\bar{z}$ \\
\end{tabular} & 产 & 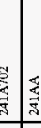 & 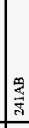 & 要 & 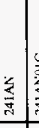 & 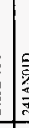 & & 蒿 & 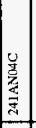 & 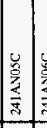 & & & 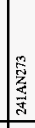 \\
\hline 罗 & & & & & & & & & & & & & & & & & & & & & & & & & & & & & \\
\hline 鸢㟟 & 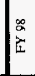 & $\approx$ & 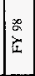 & \begin{tabular}{|l|}
2 \\
5 \\
5
\end{tabular} & $\frac{2}{2}$ & Z & F & & & & & & 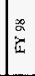 & \begin{tabular}{|l|}
$\infty$ \\
0 \\
$E$ \\
$E$
\end{tabular} & & 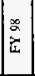 & $\frac{\alpha}{E}$ & tr & & 器 & 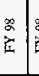 & & & F & 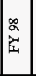 & 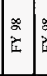 & 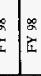 & $\approx$ & F \\
\hline
\end{tabular}

C-11 


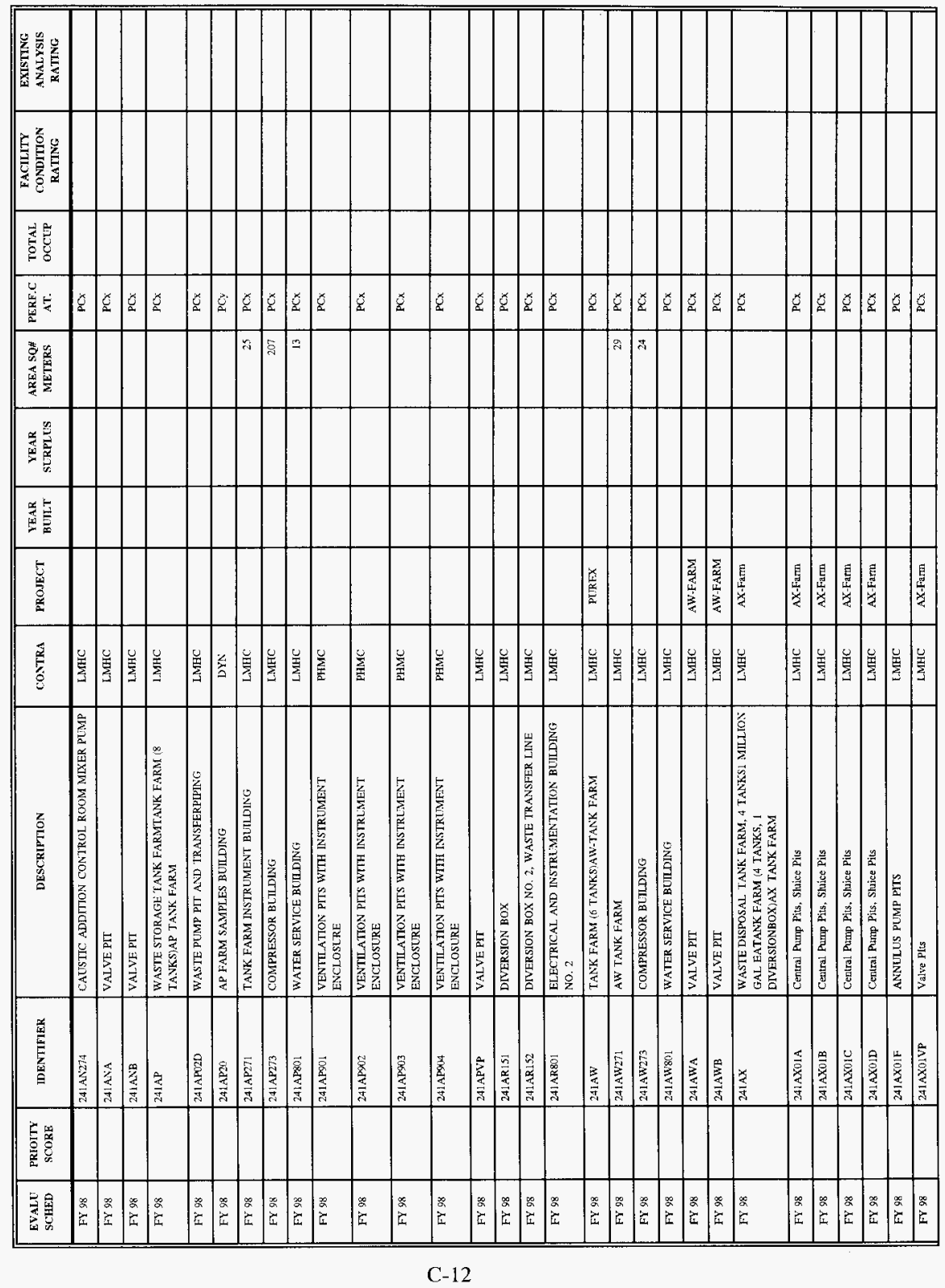




\begin{tabular}{|c|c|c|c|c|c|c|c|c|c|c|c|c|}
\hline $\begin{array}{l}\text { EVALU } \\
\text { SCHED }\end{array}$ & $\begin{array}{l}\text { PRIOITY } \\
\text { SCORE }\end{array}$ & IDENTIFJER & DESCRIPTION & CONTRA & PROJECT & $\begin{array}{l}\text { YEAR } \\
\text { BETLT }\end{array}$ & $\begin{array}{l}\text { YEAR } \\
\text { SURPLLS }\end{array}$ & $\begin{array}{l}\text { AREA SQ; } \\
\text { METERS }\end{array}$ & $\begin{array}{l}\text { PERF.C } \\
\text { AT. }\end{array}$ & $\begin{array}{l}\text { TOTAL } \\
\text { OCCUP }\end{array}$ & $\begin{array}{l}\text { FACILITY } \\
\text { CONDITION } \\
\text { RATING }\end{array}$ & $\begin{array}{l}\text { EXISTING } \\
\text { ANALYSIS } \\
\text { RATING }\end{array}$ \\
\hline FY 98 & & $241 \mathrm{AX} 02 \mathrm{~B}$ & Shice Pit & LMHC & $\mathrm{AX}$-Fam & & & & $\mathrm{PCx}$ & & & \\
\hline FY 98 & & $241 \mathrm{AXO2C}$ & Shice Pit & LMHC & AX-Farm & & & & $\mathrm{PCx}$ & & & \\
\hline FY 98 & & $241 \mathrm{~A} \times 02 \mathrm{D}$ & Shice Pil & LMHC & AX-Fam & & & & $\mathrm{PCx}$ & & & \\
\hline FY 98 & & $241 \mathrm{AX} 02 \mathrm{~F}$ & ANNULUS PUMP PITS & LMHC & & & & & $\mathrm{PCK}$ & & & \\
\hline FY 98 & & $241 \mathrm{AX} 02 \mathrm{VP}$ & Valve PL:s & LMHC & AX-Famm & & & & $\mathrm{PCK}$ & & & \\
\hline FY 98 & & $241 \mathrm{AX} 03 \mathrm{~B}$ & Shice Pit & LMHC & AX-Famn & & & & $\mathrm{PCX}$ & & & \\
\hline FY 98 & & $241 \mathrm{AXO3C}$ & Shice Pit & LMHC & AX-Farm & & & & $\mathrm{PCx}$ & & & \\
\hline FY $₫ 8$ & & $241 \mathrm{AX} 03 \mathrm{D}$ & Shice Pit & LMHC & AX-Fann & & & & $\mathrm{PCx}$ & & & \\
\hline FY 98 & & $241 \mathrm{AX0} 03 \mathrm{VP}$ & Valve PIts & LMHC & AX-Famn & & & & $\mathrm{PCx}$ & & & \\
\hline FY 98 & & $241 \mathrm{~A} \times 04 \mathrm{~B}$ & Sluice Pit & LMHC & AX-Farn & & & & $\mathrm{PCx}$ & & & \\
\hline FY 98 & & $241 \mathrm{AX} 04 \mathrm{C}$ & Shrice Pit & LMHC & AX-Fam & & & & $\mathrm{PCX}$ & & & \\
\hline FY 98 & & $241 \mathrm{~A} \times 04 \mathrm{D}$ & Strice Pît & LMHC & AX.Farn & & & & $\mathrm{PCX}$ & & & \\
\hline FY 98 & & $241 \mathrm{AX} 04 \mathrm{VP}$ & Valve Plts & LMHC & AX-Farm & & & & $\mathrm{PCX}$ & & & \\
\hline FY 98 & & $241 \mathrm{AX}[5]$ & DIVERTER STATIONS & LMHC & AX-Farm & & & & $\mathrm{PC} x$ & & & \\
\hline FY 98 & & $241 \mathrm{AX} 152$ & DVNERTER STATION & LMHC & & & & & $\mathrm{PC} x$ & & & \\
\hline FY 98 & & $241 \mathrm{AX} 153$ & DWERSION BOXES & LMHC & AX-Fann & & & & $\mathrm{PCx}$ & & & \\
\hline FY 98 & & $241 \mathrm{AX} 155$ & DFERSION BOK & LMHC & & & & & $\mathrm{PCx}$ & & & \\
\hline FY 98 & & $241 \mathrm{AX} \times 401$ & DNERTER PIT & LMHC & & & & & $\overline{P C x}$ & & & \\
\hline FY 98 & & $241 \mathrm{AX} 501$ & CONDENSATE VALVE PIT & LMHC & & & & 514 & $\mathrm{PCx}$ & & & \\
\hline FY 98 & & $241 \mathrm{~A} \times 80$ & A COMPLEX SERVICE BUTIDING & ЕМНС & & & & & $\mathrm{PC} x$ & & & \\
\hline FY 98 & & $241 \mathrm{AX} 801 \mathrm{~A}$ & TANK FARM CONTROL HOUSE, NORTH & LMHC & AX-Farmi & & & 27 & $\mathrm{PCx}$ & & & \\
\hline FY 98 & & $241 \mathrm{~A} \times 801 \mathrm{~B}$ & TANK FARM CONTROL HOUSE, SOLTH & LMHC & AX-FamI & & & 27 & $\mathrm{PCx}$ & & & \\
\hline FY 98 & & $241 \mathrm{AX} 801 \mathrm{C}$ & $\begin{array}{l}\text { TAAK FARM DTVERSION HOUSE, SOUTH OF A } \\
\text { FARM }\end{array}$ & LMHC & & & & 7 & $\mathrm{PCX}$ & & & \\
\hline FY 98 & & 241AXA PTा & VALVE PIT & LMHC & & & & & $\mathbf{P C x}$ & & & \\
\hline FY 98 & & 241AXB PIT & VALVE PIT & LMHC & & & & & $\mathrm{PCx}$ & & & \\
\hline FY 98 & & $241 \mathrm{AY}$ & $\begin{array}{l}\text { WASIE DISPOSAL IANK FARM. } 2 \text { TANKS1 MGAL } \\
\text { EACHTANK FARM (2 TANKS, } 2 \\
\text { DIVERSIONBOXESIAY TANK FARM }\end{array}$ & LMHC & PUREX & & & & $\mathrm{PCX}$ & & & \\
\hline FY 98 & & 24:AY01B & SLUUICE PTT & LMHC & AY-FARM & & & & $\mathrm{PCx}$ & & & \\
\hline FY 98 & & 24IAYOIC & SLUICE PTT & LMHC & AY-FARM & & & & $\mathbf{P C x}$ & & & \\
\hline FY 98 & & 24IAYOLD & SLUICE PTT & LMHC & AY-FARM & & & & $\mathbf{P C x}$ & & & \\
\hline FY 98 & & 241AY0IE & SLUICE PIT & LMHC & AY-FARM & & & & $\mathrm{PCX}$ & & & \\
\hline FY 98 & & $241 \mathrm{AYOIF}$ & ANNULUS PUMP PIT & LMHC & AY-FARM & & & & $\mathrm{PCX}$ & & & \\
\hline FY 98 & & $241 \mathrm{AY} 02 \mathrm{~B}$ & SLUTCE PIT & LMHC & AY-FARM & & & & $\mathrm{PCx}$ & & & \\
\hline FY 98 & & $241 \mathrm{AY} 02 \mathrm{C}$ & SLUTCE PTT & L.MHC & AY-FARM & & & & $\mathrm{PCx}$ & & & \\
\hline
\end{tabular}




\begin{tabular}{|c|c|c|c|c|c|c|c|c|c|c|c|c|}
\hline $\begin{array}{l}\text { EVALU } \\
\text { SCHED }\end{array}$ & $\begin{array}{l}\text { PRIOITY } \\
\text { SCOKE }\end{array}$ & IDENTIFIER & DESCRIPTION & CONTRA & PROJECT & $\begin{array}{l}\text { YEAR } \\
\text { BUILT }\end{array}$ & $\begin{array}{c}\text { YEAR } \\
\text { SURPLUS }\end{array}$ & $\begin{array}{l}\text { AREA SQF } \\
\text { METERS }\end{array}$ & $\begin{array}{l}\text { PERF.C } \\
\text { AT. }\end{array}$ & $\begin{array}{l}\text { TOTAL } \\
\text { OCCTP }\end{array}$ & $\begin{array}{l}\text { FACILITY } \\
\text { CONDITION } \\
\text { RATING }\end{array}$ & $\begin{array}{c}\text { EXISTING } \\
\text { ANALYSIS } \\
\text { RATING }\end{array}$ \\
\hline FY 98 & & 241AY02D & SLUTCE PT & ¿MHC & AY-FARM & & & & $\mathrm{PCx}$ & & & \\
\hline FY 98 & & 241AYO2E & SLUICE PIT & LMHC & AY-FARM & & & & $\mathrm{PCx}$ & & & \\
\hline FY 98 & & 241AYO2F & A.NNULUS PUMP PTT & EMHC & AY-FARM & & & & $\mathrm{PCx}$ & & & \\
\hline FY 98 & & $241 \mathrm{AY} 101 \mathrm{~A}$ & ENCASEMENT DUAL LEAK DETECTION PIT & LMHC & AY-FARM & & & & $\mathrm{PC} x$ & & & \\
\hline FY98 & & $241 \mathrm{AY} 101 \mathrm{~B}$ & ENCASEMENT DUAL LEAK DETEC TION PIT & LMHC & AY-FARM & & & & PCx & & & \\
\hline FY 98 & & $241 \mathrm{AY} 102 \mathrm{~A}$ & LEAK DEIECTION PIT & LMHC & AY-FARM & & & & $\mathrm{PCx}$ & & & \\
\hline FY 98 & & $241 \mathrm{AY} \mid 51$ & PUMP OUT PETS & LMHC & & & & & PCx & & & \\
\hline $\mathrm{FY} 98$ & & 241AY152 & SLLICE TRANSFER BOXES & LMHC & & & & & PCx & & & \\
\hline$F Y 98$ & & $241 \mathrm{AY} 401$ & $\begin{array}{l}\text { VENT RECIRCULATTON EQLTPMENTVAULTVENT } \\
\text { RECIRCULATION EQUPMENTVAULT }\end{array}$ & LMHC & & & & 37 & $\mathrm{PC} x$ & & & \\
\hline $\mathrm{FY} 98$ & & 241 AY402 & VENT RECIRCULATION EQUTPMENT & LMHC & & & & 37 & $\mathrm{PCx}$ & & & \\
\hline FY 98 & & $241 \mathrm{AY} 501$ & VALVE PTT & LMHC & & & & & PCx & & & \\
\hline FY 98 & & $241 \mathrm{AY} 51$ & EI.ECTRICAL EQUIPMENT BLILDING 24 2L-C.106 & LMHC & & & & & $\mathrm{PC} x$ & & & \\
\hline FY 98 & & $241 \mathrm{AY} 51 \mathrm{~A}$ & SEISYIC SHUTDOWN SYSTEM 1A \& 241-C-106 & LMHC & & & & & $\mathrm{PCx}$ & & & \\
\hline FY 98 & & $241 \mathrm{AY} 51 \mathrm{~B}$ & SEISMIC SHUTDOWN SYSTEM IB 4 G 24-C-106 & LMHC & & & & & $\mathrm{PCx}$ & & & \\
\hline FY 98 & & $241 \mathrm{AY} 801$ & TANK FARM INSTRUMENT HOUSE & PHMC & & & & 37 & $\mathrm{PCx}$ & & & \\
\hline FY 98 & & $241 \mathrm{AYYD} 1 \mathrm{~A}$ & AY TANK FARM & LMHC & & & & & $\mathrm{PCx}$ & & & \\
\hline FY 98 & & $241 \mathrm{AZ}$ & $\begin{array}{l}\text { WASTE DISPOSAL TANK FARM, } 2 \text { TANKS1 MGAL } \\
\text { EACHTANK FARM (2 TANKS)AZ TANK FARM }\end{array}$ & LMHC & PCREX & & & & $\mathrm{PCx}$ & & & \\
\hline FY 98 & & $241 \mathrm{AZO1A}$ & VALVE PIT & LMHC & & & & & $\mathrm{PCx}$ & & & \\
\hline FY 98 & & $241 \mathrm{AZO1B}$ & VALVE PIT & LMHC & & & & & $\mathrm{PCX}$ & & & \\
\hline FY 98 & & 241AZOLC & VALVE PIT & LMHC & & & & & $\mathrm{PCx}$ & & & \\
\hline FY 98 & & $241 \mathrm{AZZ151}$ & DIVERTER STATION & LMHC & & & & & PCX & & & \\
\hline FY 98 & & $24 ! \mathrm{AZ} \mid S 2$ & SLUICE IRANSFER BOX & LMHC & & & & & $\mathrm{PCx}$ & & & \\
\hline FY 98 & & 24!AZ153 & CONDENSATE VALVE PIT & LMHC & & & & & $\mathrm{PCx}$ & & & \\
\hline FY 98 & & $241 \mathrm{AZ} 154$ & CONDENSATE PUMP PIT & LMHC & & & & & $\mathrm{PCx}$ & & & \\
\hline FY 98 & & $24 ! A Z 155$ & CONTAMINATED STORAGE PIT & LMHC & & & & & $\mathrm{PCx}$ & & & \\
\hline FY 98 & & $241 \mathrm{AZ} 156$ & MIXER PIMP SPEED CONTROLLER & LMHC & & & & & $\mathrm{PCx}$ & & & \\
\hline FY 98 & & $241 \mathrm{AZ271}$ & CHANGE HOUSE/CONTROL BUILING & LMHC & & & & 459 & $\mathrm{PCx}$ & & & \\
\hline FY 98 & & $241 \mathrm{AZ} 401$ & VENT RECIRCLLATION EQUIPMENT VAULT & LMHC & & & & 37 & $\mathrm{PCx}$ & & & \\
\hline FY 98 & & $241 \mathrm{AZ402}$ & VENT RECLRCULATION EQUIPMENT VAULT & LMHC & & & & 37 & $\mathrm{PCK}$ & & & \\
\hline FY 98 & & $241 \mathrm{AZ701}$ & $\begin{array}{l}\text { STANDBYGENERATOR/COMPRESSOR/SERVICEBLD } \\
\text { GSTANDBY GEN/COMP/SERVICE }\end{array}$ & LMHC & & & & & $\mathrm{PCx}$ & & & \\
\hline FY 98 & & $241 \mathrm{AZ7} 702$ & WASTE TANK VENTILATION BUILDING & LMHC & & & & 929 & $\mathrm{PCx}$ & & & \\
\hline FY 98 & & $241 \mathrm{AZ} 801$ & EP BLDG & LMHC & & & & 36 & $\mathrm{PCx}$ & & & \\
\hline FY 98 & & $241 \mathrm{AZZON} 1 \mathrm{~A}$ & AZ TANK FARM & LMHC & & & & 55 & $\mathrm{PCx}$ & & & \\
\hline
\end{tabular}




\begin{tabular}{|c|c|c|c|c|c|c|c|c|c|c|c|c|}
\hline $\begin{array}{l}\text { EVALU } \\
\text { SCHED }\end{array}$ & $\begin{array}{l}\text { PRloITY } \\
\text { sCORE }\end{array}$ & IDENTIFIER & DESCRIPTION & CONTRA & PROJECT & $\begin{array}{l}\text { YEAR } \\
\text { BLILT }\end{array}$ & $\begin{array}{l}\text { YEAR } \\
\text { SURPLCS }\end{array}$ & $\begin{array}{l}\text { AREA SQ\# } \\
\text { METERS }\end{array}$ & $\begin{array}{c}\text { PERT.C } \\
\text { AT. }\end{array}$ & $\begin{array}{l}\text { Total } \\
\text { OCCUP }\end{array}$ & $\begin{array}{l}\text { FACILITY } \\
\text { CONDITION } \\
\text { RATING }\end{array}$ & $\begin{array}{l}\text { EXISTING } \\
\text { ANALYSIS } \\
\text { RATING }\end{array}$ \\
\hline FY 98 & & $24: \mathrm{B}$ & 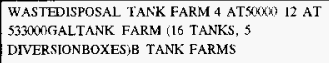 & LMHC & & & & & $\mathrm{PCx}$ & & & \\
\hline FY 98 & & $241 \mathrm{~B} 151$ & DNERSION ROXES & LMHC & В.Fалп & & & & $\mathbf{P C x}$ & & & \\
\hline FY 98 & & $241 \mathrm{~B} 152$ & DIVERSION BOXES & LMEC & B-Farm & & & & $\mathrm{PCx}$ & & & \\
\hline FY 98 & & $241 \mathrm{~B} 153$ & DTVERSTON BOXES & LMHIC & B-Farm & & & & $\mathrm{PCx}$ & & & \\
\hline FY 98 & & $241 \mathrm{~B} 154$ & DIVERSION BOXES & LMHC & B-Fam & & & & $\mathrm{PCx}$ & & & \\
\hline FY 98 & & $241 \mathrm{~B} 252$ & DIVERSION BOX & LMHC & B-Famm & & & & $\mathrm{PCX}$ & & & \\
\hline FY 98 & & $241 \mathrm{~B} 701$ & INSTRLMENT AIR COMPRESSORBUILDING & LMHC & B.Farm & & & 5 & $\mathrm{PCK}$ & & & \\
\hline FY 98 & & $24 \mathrm{IBR}$ & $\begin{array}{l}\text { WASTE METAL RECOVERY FACILITIES } \\
\text { BFARMCBSII SUBSTATION }\end{array}$ & L.MHC & & & & & $\mathrm{PCx}$ & & & \\
\hline FY 98 & & $241 \mathrm{BR} 152$ & DIVERSION BOXES & LMHC & & & & & $\mathrm{PCx}$ & & & \\
\hline FY 98 & & $24 ! \mathrm{BK}$ & $\begin{array}{l}\text { 12TANKS AT } 533000 \text { GAL EATANK FARM (12 } \\
\text { TANKS, } 6 \text { DNERSTONBOXES)BX TANK FARM }\end{array}$ & LMHC: & & & & & PCK & & & \\
\hline FY 98 & & $24 \mathrm{lBX} 04$ & Central Pump Pits & LMHC & BX-Fann & & & & $\mathrm{PCx}$ & & & \\
\hline FY 98 & & $24 \mathrm{LBX} 07$ & Central Punp Pits & LMHC & BX-Farn & & & & $\mathrm{PCX}$ & & & \\
\hline FY 98 & & $24 \mathrm{iBX} 08$ & Central Pump Pits & LMHC & BX-Famn & & & & $\mathrm{PCX}$ & & & \\
\hline FY 98 & & $24 \operatorname{lB} \times 09$ & Ceniral Pump Pits & LMEC: & BX-Farm & & & & $\mathrm{PCK}$ & & & \\
\hline FY 98 & & $241 \mathrm{~B} \times 10$ & Central Pump Pits & LMHC & BX-Farm & & & & PCx & & & \\
\hline FY 98 & & $241 \mathrm{~B} \times 11$ & Central Rimp Pits & LМHC & BX-Farn & & & & $\mathrm{PCx}$ & & & \\
\hline FY 98 & & $24 \mathrm{IRX} 12$ & Cenitral Fump Pits & LMHC & BX-Farm & & & & $\mathrm{PCx}$ & & & \\
\hline FY 98 & & $241 \mathrm{BX} 153$ & DTVERSON BOXES & LMHC & & & & & $\mathrm{PCx}$ & & & \\
\hline FY 98 & & $241 \mathrm{BX} 154$ & DNVERSION BOXES & LMHC & & & & & $\mathrm{PCx}$ & & & \\
\hline FY 98 & & $241 \mathrm{BX} 155$ & DTVERSION BOXES & LMHC & & & & & $\mathrm{PCx}$ & & & \\
\hline FY 98 & & $241 \mathrm{~B} \times 2.4$ & INSTRUMENT BUILDEG & LMHC & & & & & $\mathrm{PCx}$ & & & \\
\hline FY 98 & & 241BXR151 & DIVERSION BOXESDIVERSION BOX & LMHC & BX-Farn & & & & $\mathrm{PCX}$ & & & \\
\hline FY 98 & & $241 \mathrm{BXR152}$ & DIVERSION BOXESDIVERSION BOX & LMHC & BX-Farm & & & & $\mathrm{PCx}$ & & & \\
\hline FY 98 & & $241 \mathrm{BXR153}$ & $\begin{array}{l}\text { WST MIL RECOVERY FACILITIES, DIVERSION } \\
\text { BOXESONVERSION BOX }\end{array}$ & LMHC & BX-Fann & & & & $P C x$ & & & \\
\hline FY 98 & & $241 \mathrm{BY}$ & $\begin{array}{l}\text { WASTE DISPOSAL TANK FARM }(12 \text { TANKS, } 3 \\
\text { DNERSION BOXES)BY TANK FARM }\end{array}$ & LMHC & & & & 96 & $\mathrm{PCX}$ & & & \\
\hline FY 98 & & 241BY01A & Central Purg Pils & LMHC & BY-Farm & & & & $\mathrm{PCx}$ & & & \\
\hline FY 98 & & $241 \mathrm{BYO} 1 \mathrm{~B}$ & Shice Pits & LMHC & BY-Fam & & & & $\mathrm{PCX}$ & & ' & \\
\hline FY 98 & & $24 \mathrm{IBY} 01 \mathrm{C}$ & Shice Pits & LMHC & BY-Farm & & & & $\mathrm{PCX}$ & & & \\
\hline FY 98 & & $241 \mathrm{BY} 01 \mathrm{D}$ & Shice Pits & LMHC & BY-Famn & & & & $\mathrm{PCX}$ & & & \\
\hline FY 98 & & $241 \mathrm{BY} 02 \mathrm{~A}$ & Central Pump Pits & LMHC & BY-Famn & & & & $\mathrm{PCX}$ & & & \\
\hline FY 98 & & $241 \mathrm{BY} \cup 2 \mathrm{~B}$ & Shice Pits & LMHC & BY-Fanin & & & & $\mathrm{PCx}$ & & & \\
\hline
\end{tabular}




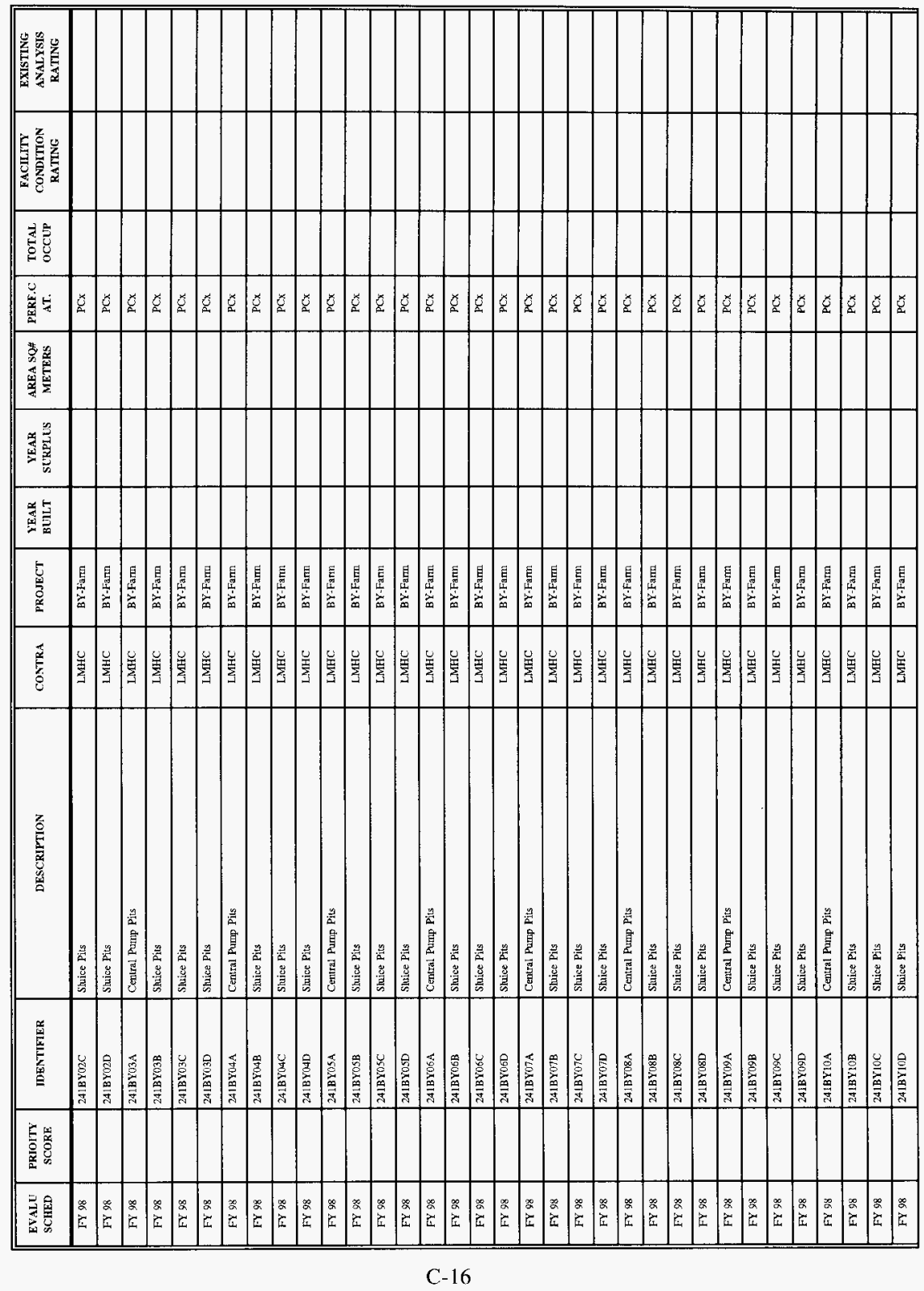




\begin{tabular}{|c|c|c|c|c|c|c|c|c|c|c|c|c|}
\hline $\begin{array}{l}\text { EVALU } \\
\text { SCHED }\end{array}$ & $\begin{array}{l}\text { PRIOITY } \\
\text { SCORE }\end{array}$ & IDENTIFIER & DESCRIPTION & CONTRA & PROJECT & $\begin{array}{l}\text { YEAK } \\
\text { BUILT }\end{array}$ & $\begin{array}{l}\text { YEAR } \\
\text { SURPLUS }\end{array}$ & $\begin{array}{l}\text { AKEA SQ\# } \\
\text { METERS }\end{array}$ & $\begin{array}{l}\text { FERF.C } \\
\text { AT. }\end{array}$ & $\begin{array}{l}\text { TOTAL } \\
\text { OCCUP }\end{array}$ & $\begin{array}{l}\text { FACILITY } \\
\text { CONDITION } \\
\text { RATING }\end{array}$ & $\begin{array}{l}\text { EXISTING } \\
\text { ANALYSIS } \\
\text { RATING }\end{array}$ \\
\hline FY 98 & & $241 \mathrm{BY} 11 \mathrm{~A}$ & Central. Pump Pils & LMHC & BY-Fann & & & & $\mathrm{PCx}$ & & & \\
\hline FY 98 & & $24\lfloor$ BY11B & Shuice Pits & LMHC & BY-Farm & & & & $\mathrm{PCx}$ & & & \\
\hline FY 98 & & 24 BYIIC & Stuice Pits & LMHC & BY.Farmi & & & & $\mathrm{PCx}$ & & & \\
\hline FY 98 & & $241 \mathrm{BY} I I D$ & Stuice Pits & LMHC & BY-Fami & & & & $\mathrm{PCx}$ & & & \\
\hline FY 98 & & $241 \mathrm{BY} 12 \mathrm{~A}$ & Central Punt Pits & LMHC & BY-Faml & & & & $\mathrm{PCx}$ & & & \\
\hline FY 98 & & 241BY12B & Sluies Pits & LMHC & BY-Farm & & & & $\mathrm{PCX}$ & & & \\
\hline FY 98 & & 241BY12C & Shuicc Pits & LMHC & BY-Fann & & & & $\mathrm{PCx}$ & & & \\
\hline FY 98 & & $241 \mathrm{BY} 12 \mathrm{D}$ & Stuice Pits & LMHC & BY-Farm & & & & $\mathrm{PCx}$ & & & \\
\hline FY 98 & & $241 \mathrm{BY} 254$ & CONTROL. HOUSE AVD COMPRESSOR,ITS2 & LMHC & BY-Fatm & & & 68 & $\mathrm{PCx}$ & & & \\
\hline FY 98 & & $241 \mathrm{BY} 301$ & $\begin{array}{l}\text { CONTROL HOUSE IN TANKSOL.IDIFICATTONS } \\
\text { ITSIINSTRUMENT CONTROL }\end{array}$ & LMHC & BY-Fann & & & 10 & $\mathrm{PCx}$ & & & \\
\hline$\overline{F Y} 98$ & & $241 \mathrm{BY} 302$ & COMPRESSOR HOLSE, ITSI & LMHC & BY-Farm & & & 94 & $\mathrm{PCx}$ & & & \\
\hline FY 98 & & $241 \mathrm{BY} 302 \mathrm{~A}$ & $\begin{array}{l}\text { COMPRESSED AIR. STATONCOMPRESSED AIR } \\
\text { STATION }\end{array}$ & LMHC & & & & & $\mathrm{PCx}$ & & & \\
\hline FY 98 & & $241 \mathrm{BYR}$ & WASTE METAL RECOVERY FACILITIES,BY FARM & LMHC & & & & & $\mathrm{PCx}$ & & & \\
\hline FY 98 & & $241 \mathrm{BYR} 152$ & DIVERSION BOXES & LMHC & BY-Farm & & & & $\mathrm{PCx}$ & & & \\
\hline$\overline{\text { FY } 98}$ & & $241 \mathrm{BYR} 153$ & DIVERSION BOXES & LMHC & BY.Fann & & & & $\mathrm{PCx}$ & & & \\
\hline$\overline{F Y} 98$ & & 241 BYRI $\$ 4$ & DIVERSION BOXES & LMHC & BY-Fann & & & & $\mathrm{PCx}$ & & & \\
\hline$\overline{F Y} 98$ & & 241BYTK2 & SOAK/RLNSE TANK & LMHC & & & & & $\mathrm{PCx}$ & & & \\
\hline FY 98 & & $241 \mathrm{BYTK} 302 \mathrm{C}$ & ACD NEUTRALIZATION TANK & LMHC & & & & & $\mathrm{PCx}$ & & & \\
\hline FY 58 & & $241 \mathrm{C}$ & $\begin{array}{l}\text { TANK FARMS } \text { i16 TANKS, } 6 \\
\text { DIVERSIONBOXES;C-TANK FARM }\end{array}$ & LMHC & PLREX & & & & $\mathbf{P C x}$ & & & \\
\hline FY $\$ 8$ & & $241 \mathrm{CO} 2 \mathrm{~A}$ & CENTRAL PUMP PITS & LMHC & C.Farm & & & & $\mathrm{PCx}$ & & & \\
\hline FY 58 & & $241003 \mathrm{~A}$ & CENTRAL PUMP PLTS & LMHC & C.Fan & & & & $\mathrm{PCx}$ & & & \\
\hline FY 98 & & $241 \mathrm{CO4A}$ & CENTRAL PUMP PITS & LMHC & C-Fanm & & & & $\mathrm{PCx}$ & & & \\
\hline$\overline{F Y} 98$ & & $241 \cos \mathrm{A}$ & CENIRAL PUMP PITS & LMHC & C.Fam & & & & $\mathrm{PCx}$ & & & \\
\hline FY 98 & & $241 \mathrm{C} 06 \mathrm{~A}$ & CENTRAL PUMP PITS & LMHC & C-Farm & & & & $\mathrm{PCX}$ & & & \\
\hline FY 98 & & $241 C 151$ & DNERSION BOXES & LMHC & C-Fann & & & & $\mathrm{PCx}$ & & & \\
\hline FY 98 & & $241 \mathrm{Cl} 52$ & DIVERSION BOXES & LMHC & C.Fam & & & & $\mathrm{PCx}$ & & & \\
\hline FY 98 & & $241 \mathrm{Cl} 53$ & DNERSION BOXES & LMHC & C-Farm & & & & $\mathrm{PCx}$ & & & \\
\hline FY 98 & & $241 \mathrm{C} 154$ & DIVERSION BOXES & LMHC & C-Farm & & & & $P C x$ & & & \\
\hline$\overline{F Y} 98$ & & $241 \mathrm{C} 252$ & DIVERSION BOXES & LMHC & C-Farm & & & & $\mathbf{P C X}$ & & & \\
\hline FY 98 & & $241 \mathrm{CS} 1$ & ELECTRICAL EQUIPMENT \& 241-C-106 & LMAC & & & & & $\mathrm{PCX}$ & & & \\
\hline FY 98 & & $241 \mathrm{C} 51 \mathrm{~A}$ & SEISMIC SHLTDOWN SYSTEM IA \& 241-C-106 & LMAC & & & & & PCX & & & \\
\hline FY 98 & & $241 C 51 B$ & SEISMIC SHUTDOWN SYSTEM IB @ $241 \cdot \mathrm{C}-105$ & LMHC & & & & & $\mathrm{PCx}$ & & & \\
\hline
\end{tabular}




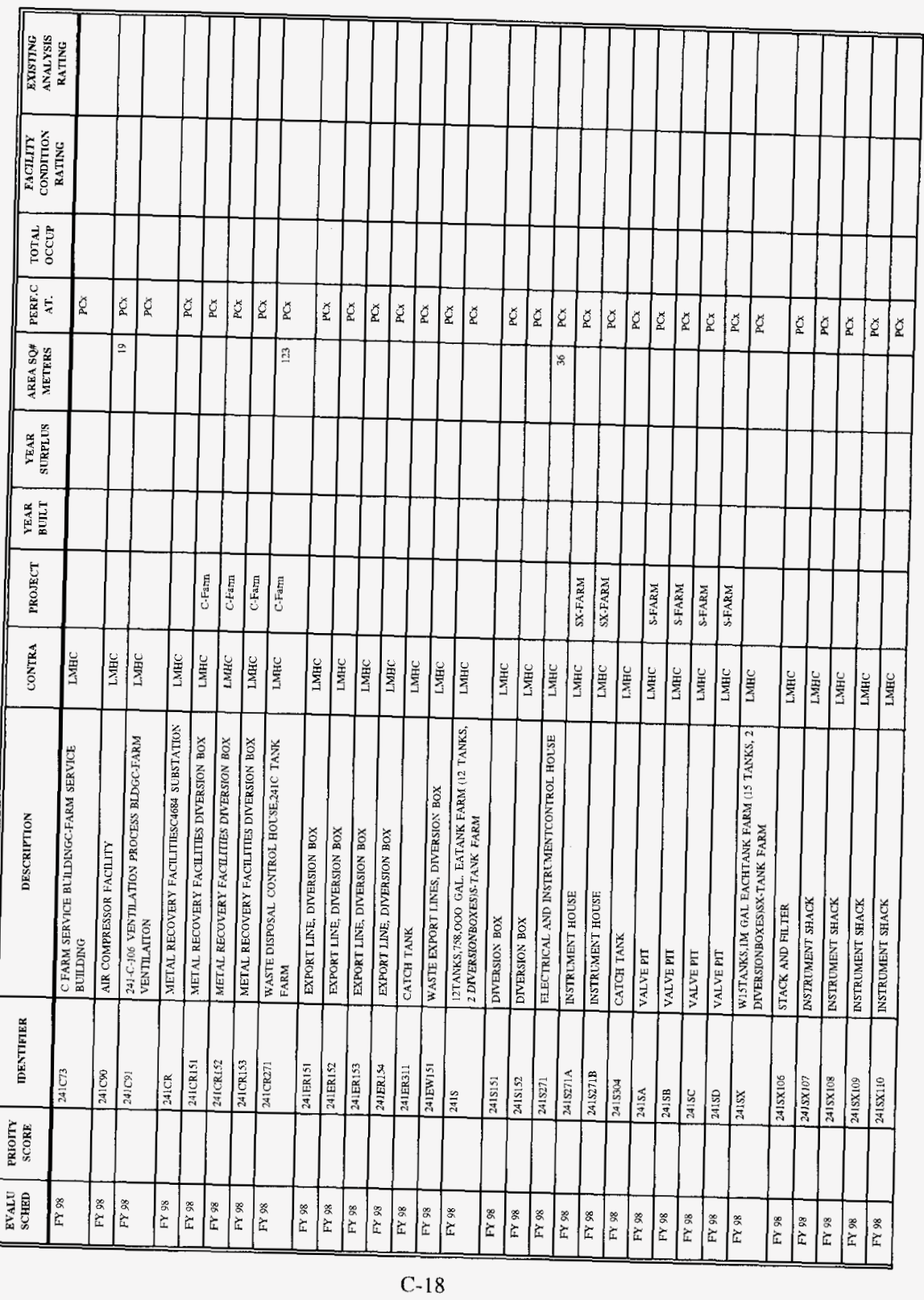




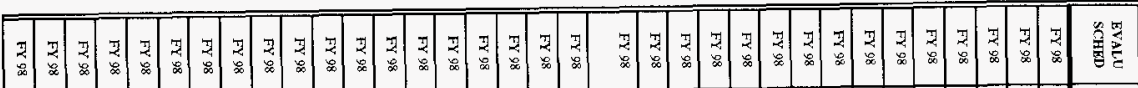

设

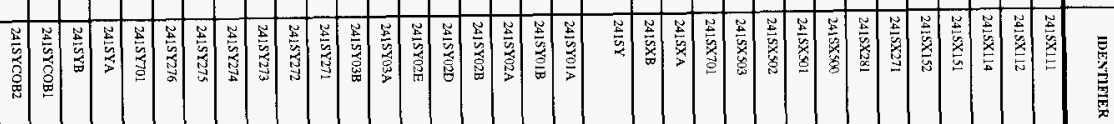

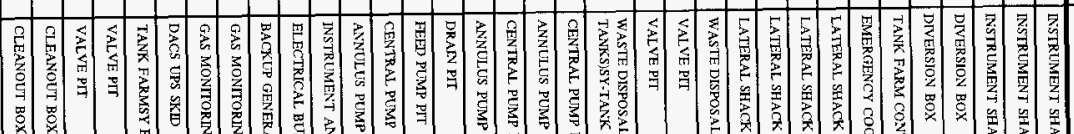

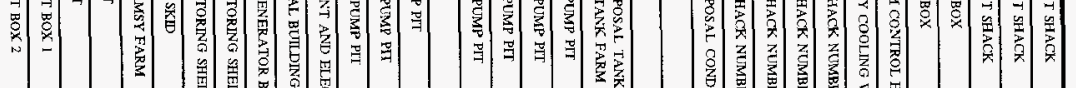

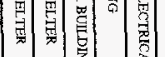

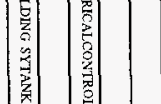

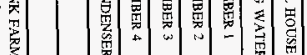

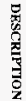

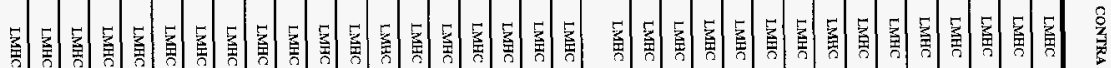

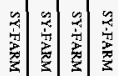

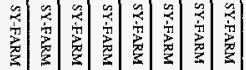

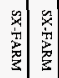

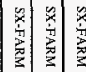

衷

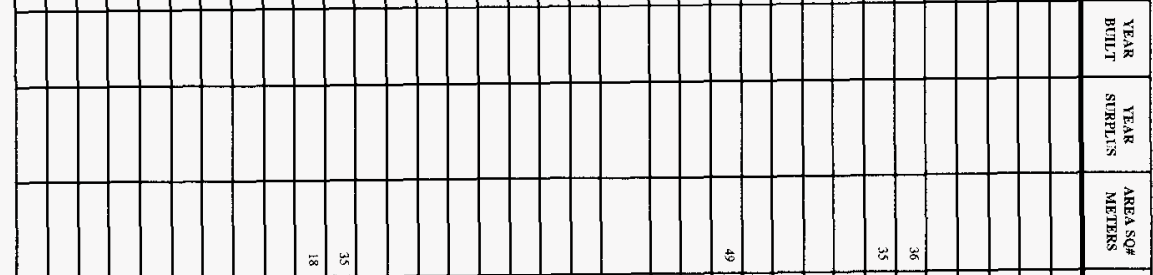

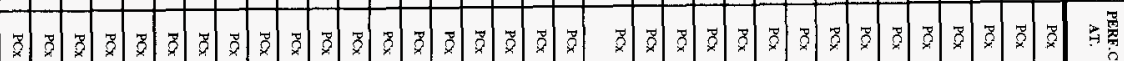

\begin{tabular}{|ll}
\hline & \\
\hline
\end{tabular}




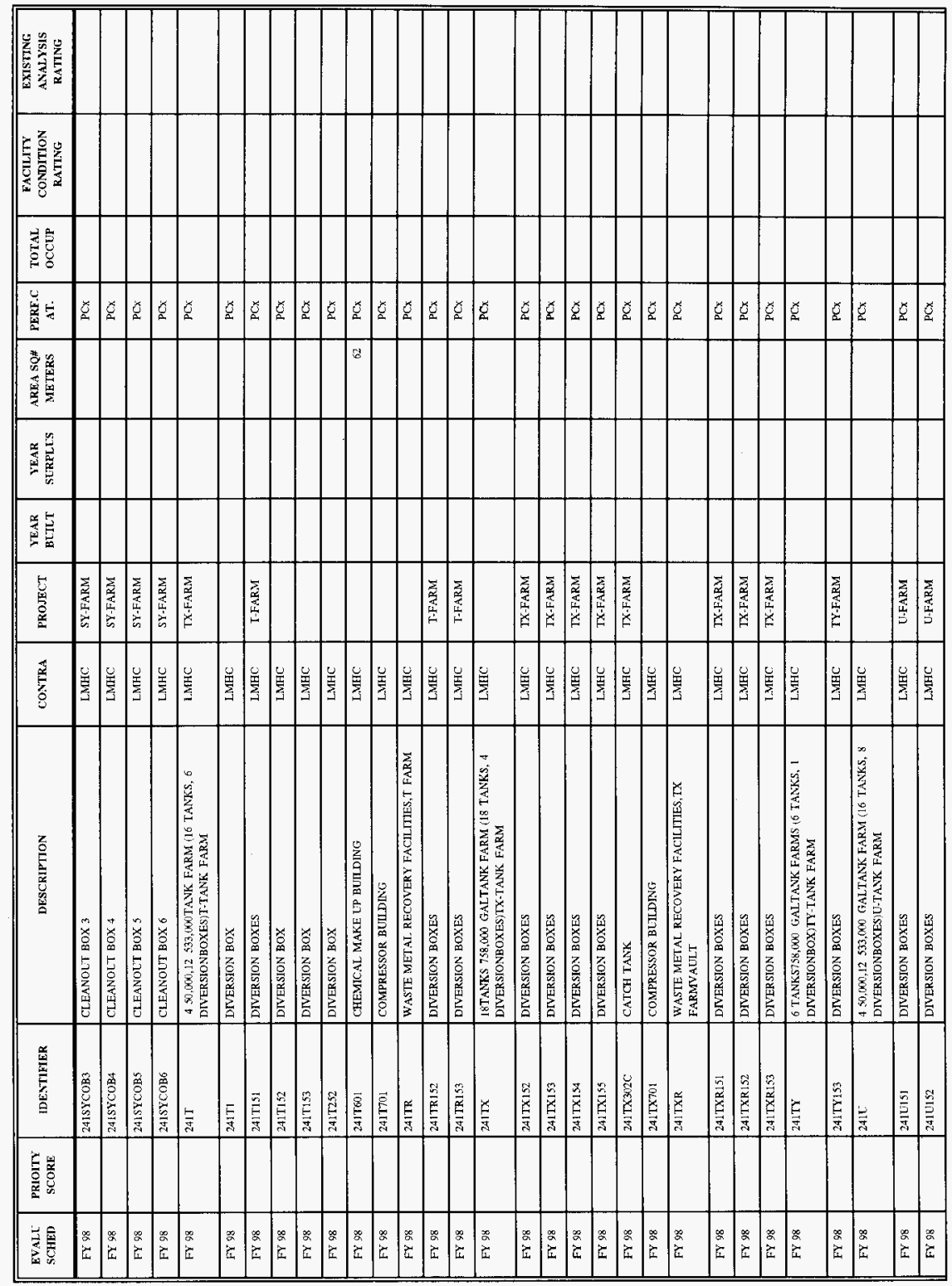




\begin{tabular}{|c|c|c|c|c|c|c|c|c|c|c|c|c|}
\hline $\begin{array}{l}\text { EYALU } \\
\text { SCHED }\end{array}$ & $\begin{array}{l}\text { PRIOITY } \\
\text { SCORE }\end{array}$ & IDENTIFER & DESCRIPTION & CONTRA & PKOJECT & $\begin{array}{l}\text { YEAR } \\
\text { BUILT }\end{array}$ & $\begin{array}{c}\text { YEAR } \\
\text { SLERLLS }\end{array}$ & $\begin{array}{l}\text { AREA SQ\# } \\
\text { METERS }\end{array}$ & $\begin{array}{c}\text { PERF.C } \\
\text { AT. }\end{array}$ & $\begin{array}{l}\text { TOTAL } \\
\text { OCCUP }\end{array}$ & $\begin{array}{l}\text { FACILITY } \\
\text { CONDITION } \\
\text { RATING }\end{array}$ & $\begin{array}{l}\text { EXISTING } \\
\text { ANALYSIS } \\
\text { RATING }\end{array}$ \\
\hline $\mathrm{FY} 98$ & & $241 \mathrm{C} 153$ & DIVERSION BOXES & LMHC & U-FARM & & & & $\mathrm{PCK}$ & & & \\
\hline FY 98 & & 2411252 & DIVERSION BOXES & LMHC & U-FARM & & & & $\mathrm{PC} x$ & & & \\
\hline FY 98 & & $241 \mathrm{~L} 271$ & U FARM CONTROL HOUSE & LMHC & & & & 22 & $\mathrm{PC} x$ & & & \\
\hline FY 98 & & $241 \mathrm{C} 301$ & CATCH TANK & LMHC & & & & & $\mathrm{PCx}$ & & & \\
\hline FY 98 & & 2410791 & INSTR UMENT AIR COMPRESSOR HOLSE & LMHC & & & & 4 & $\mathrm{PCx}$ & & & \\
\hline FY 98 & & $2410 \mathrm{~A}$ & VALVE PIT & LMHC & U-FARM & & & & $\mathrm{PCx}$ & & & \\
\hline FY 98 & & $241 \mathrm{UB}$ & VALVE PIT & LMHC & L-FARM & & & & $\mathrm{PCK}$ & & & \\
\hline FY 98 & & $241 \mathrm{UC}$ & VALVE PIT & LMHC: & U-FARM & & & & $\mathrm{PCK}$ & & & \\
\hline FY 98 & & $24 \mathrm{110}$ & VALVE PIT & LMHC & L-FARM & & & & $\mathrm{PCX}$ & & & \\
\hline FY 98 & & 241LR151 & DNERSION BOXES & LMHC & L.FARM & & & & $\mathrm{PCx}$ & & & \\
\hline $\mathrm{FY} 98$ & & $241 \mathrm{LR} 152$ & DNERSION BOXES & LMHC & L:FARM & & & & $\mathrm{PC}$ & & & \\
\hline FY 98 & & 241 R153 & DNERSION BOXES & LMHC & U-FARM & & & & $\mathrm{PCx}$ & & & \\
\hline FY 98 & & 241 UR154 & DIVERSION BOXES & LMHC & U-FARM & & & & $\mathrm{PCx}$ & & & \\
\hline FY 98 & & $2410 \times 154$ & DIVERSION BOX & LMHC & U-FARM & & & & $\mathrm{PCx}$ & & & \\
\hline .FY 98 & & $241 \mathrm{CX} 302 \mathrm{~A}$ & CATCH TANK & LMHC & U-FARM & & & & $\mathrm{PCx}$ & & & \\
\hline $\mathrm{FY} 98$ & & $242 \mathrm{~A} 204$ & NIFRC ACD UNLOADING STATION & PHMC & & & & & $\mathrm{PCx}$ & & & \\
\hline FY 98 & & $242 \mathrm{~A} 3 \mathrm{X}$ & CATCH TANK & LMHC & & & & & $\mathrm{PCX}$ & & & \\
\hline $\mathrm{FY} 98$ & & $242 \mathrm{AB}$ & EVAPORATOR CONTROL BUILDNG & RFSH & & & & & $\mathrm{PCy}$ & & & \\
\hline FY 98 & & $242 \mathrm{AC}$ & PIPEFITIERS SHOP - A FARM & PHMC & & & & 74 & $\mathrm{PCx}$ & & & \\
\hline FY 98 & & 2425 & EVAPORATOR FACILITY & LMHC & & & & 766 & $\mathrm{PCx}$ & & & \\
\hline FY 98 & & $242.5302 C$ & ACD STORAGE TANK & LMHC & & & & & $\mathrm{PCx}$ & & & \\
\hline FY 98 & & 2425702 & TURRBNE BUIIDING, VENTILATION & LMHC & & & & 13 & $\mathrm{PCx}$ & & & \\
\hline FY 98 & & $242 \mathrm{~T}$ & WASTE DISPOSAI. EVAPORATOR BUUDLNG & LMHC & IX-FARM & & & 252 & $\mathrm{PCx}$ & & & \\
\hline FY 98 & & $242 \mathrm{~T} 151$ & DIVERSFON BOX & LMHC & & & & & $\mathrm{PCx}$ & & & \\
\hline FY 98 & & $242 \mathrm{T271}$ & EVAPORATOR CONTROL BIILDNG & LMHC & & & & & $\mathrm{PCx}$ & & & \\
\hline FY 98 & & $242 \mathrm{~T} 601$ & CONTROL FACILITY & LMHC & & & & 62 & $\mathrm{PCX}$ & & & \\
\hline FY 98 & & $242 \mathrm{TA}$ & VACLTWASTE RECERVING VAULT & LMHC & & & & & $\mathrm{PCx}$ & & & \\
\hline FY 98 & & $242 \mathrm{~TB}$ & VENT HOLSE & LMHC & & & & 18 & $\mathrm{PCx}$ & & & \\
\hline FY 98 & & $242 \mathrm{TC}$ & TANK FARM MICROCOMPLTER EQUIPMENT BLDG & LMHC & & & & 13 & $\mathrm{PCx}$ & & & \\
\hline FY 98 & & $243 G$ & GROUT FACILITY & LMHC & & & & 11 & $\mathrm{PCx}$ & & & \\
\hline FY 98 & & $243 \mathrm{Gl1}$ & TGE CONTROL ROOM MODULE & LMHC & & & & & $\mathrm{PCx}$ & & & \\
\hline FY 98 & & $243 \mathrm{Gl} 2$ & TGE CONTROL ROOM MODULE & LMHC & & & & & $P \subset x$ & & & \\
\hline FY 98 & & $243 \mathrm{GLA}$ & GROUT PROCESSING FACILITY MOTOR PTT & LMHC & & & & 33 & $\mathrm{PCx}$ & & & \\
\hline FY 98 & & $243 \mathrm{G} 2$ & GPF DRY BLEND HANDLING \& FEEDMODULE & LMHC & & & & 514 & $\mathbf{P C x}$ & & & \\
\hline
\end{tabular}




\begin{tabular}{|c|c|c|c|c|c|c|c|c|c|c|c|c|}
\hline $\begin{array}{l}\text { EVALU } \\
\text { SCHED }\end{array}$ & $\begin{array}{l}\text { PRIOITY } \\
\text { SCORE }\end{array}$ & IDESTIFIEK & DESCRIPTION & CONTRA & PROJECT & $\begin{array}{l}\text { YEAR } \\
\text { BLIJIT }\end{array}$ & $\begin{array}{l}\text { YEAR } \\
\text { SLRPLUS }\end{array}$ & $\begin{array}{l}\text { AREA SQ\# } \\
\text { METERS }\end{array}$ & $\begin{array}{l}\text { PERF.C. } \\
\text { AT. }\end{array}$ & $\begin{array}{l}\text { TOTAL } \\
\text { OCCUP }\end{array}$ & $\begin{array}{l}\text { FACILITY } \\
\text { CONDITION } \\
\text { RATING }\end{array}$ & $\begin{array}{l}\text { EXISTING } \\
\text { ANALYSIS } \\
\text { RATING }\end{array}$ \\
\hline FY 98 & & 24363 & GPF ADDTTIVES MODUEE & LMHC & & & & 514 & PCx & & & \\
\hline FY 98 & & 24364 & GPF CONTROL ROOM MODLLE & LMHC & & & & 19 & $\mathrm{PCx}$ & & & \\
\hline FY 98 & & 24365 & GPF STANDBY GENERATOR & LMHC & & & & 514 & $\mathrm{PC} x$ & & & \\
\hline FY 98 & & 24366 & GPF ELECTRICAL EQUIPMENT ROOM & LMHC & & & & & $\mathrm{PC} x$ & & & \\
\hline FY 98 & & 243650 & GROLT SEISMIC TRIP BULDING & I.MHC & & & & & $\mathrm{PCK}$ & & & \\
\hline FY 98 & & 24368 & GPF FILTRATION MODULE EXHALSTER MODLLE & LMHC & & & & & $\mathrm{PCK}$ & & & \\
\hline FY 98 & & 243681 & GPF CONTROL ROOMWATER SERVICE BUILDING & LMTC & & & & 13 & $\mathrm{POX}$ & & & \\
\hline FY 98 & & 243682 & $\begin{array}{l}\text { GROUT PROCESSING FAC. PRESSURE REDUCING } \\
\text { VALVE PIT }\end{array}$ & LMHC & & & & $s$ & $\mathrm{PCx}$ & & & \\
\hline FY 98 & & 24369 & GPF ELECTRICAL SUBSTATION & LMHC & & & & 514 & $\mathrm{PCK}$ & & & \\
\hline FY 98 & & 243STK: & CATCH TANK & LMHC & & & & & $\mathrm{PCx}$ & & & \\
\hline FY 98 & & $244 \mathrm{~A}$ & $\begin{array}{l}\text { WASTE VAULT AND INSTRLMENT HOUSE } \\
\text { RECENER TANK }\end{array}$ & LMHC & A-Farm & & & 11 & PCX & & & \\
\hline $\mathrm{FY} 98$ & & 244AR & $\begin{array}{l}\text { SLLDGE VALLT STORAGE AND PROCESSING } \\
\text { VAULT }\end{array}$ & LMHC & PUREX & 1965 & & 347 & $\mathrm{PC}$ & & & \\
\hline FY 98 & & 244AR1S1 & DIVERSION BOX NO. 2, WASTETRANSFER LINE & LMHC & & & & & $\mathrm{PCx}$ & & & \\
\hline $\mathrm{FY} 98$ & & 244AR701 & EMERGENCY GENERATOR BUHLDNG & LMHC & & & & 7 & $\mathrm{PCx}$ & & & \\
\hline $\mathrm{FY} 98$ & & 244AR702 & $500 \mathrm{KW}$ STANDBY GENERATORENCLOSURE & LMHC & & & & & $\mathrm{PCx}$ & & & \\
\hline $\mathrm{FY} 98$ & & 244AR712 & VALITT AIR LOCK \& LOAD OUTBUILDING & LMHC & & & & & $\mathrm{PCx}$ & & & \\
\hline $\mathrm{FY} 98$ & & 244AR715 & COMPRESSOR BUILDING & LMHC & & & & 47 & $\mathrm{PCx}$ & & & \\
\hline $\mathrm{FY} 98$ & & $244 \mathrm{BX}$ & SALI WELL RECEIVER VAULT & LMHC & BX-Farn & & & 13 & $\mathrm{PCx}$ & & & \\
\hline FY 98 & & $24 \mathrm{BXX}$ & $\begin{array}{l}\text { WASTE DISPOSAL } \\
\text { VATILT,UNDERGROUNDRECENTNG VAULT }\end{array}$ & LMHC & BX-Fanm & & & & $\mathrm{PO}$ & & & \\
\hline$F Y s 8$ & & $244 \mathrm{CR}$ & $\begin{array}{l}\text { WASTE DISPOSAL VAULT,UNDERGROLND } 241 \mathrm{C} \\
\text { TANK FARMVAULTVALLT }\end{array}$ & LMHC & C-Farrul & & & 220 & $\mathrm{PCx}$ & & & \\
\hline FY 98 & & 244CRWSI & 244.CR FRENCH DRAIN & LMHC & & & & & $\mathrm{PCx}$ & & & \\
\hline FY 58 & & 2445 & WASTE LFT STATION (VAL'LT) RECENER TANK & LMHC & S-FARM & & & 514 & $\mathbf{P C x}$ & & & \\
\hline$F Y 98$ & & $244 \$ 271^{\circ}$ & INSTRLMENT CONTROL HOUSE & LMHC & & & & 18 & $\mathrm{PCx}$ & & & \\
\hline FY 98 & & $244 \mathrm{TX}$ & $\begin{array}{l}\text { SALT WELL RECEIVER VALL TRECEIVFR } \\
\text { TANKSALT WELL RECEIVER VALLTT }\end{array}$ & LMHC & TX-FARM & & & 88 & $\mathrm{PCx}$ & & & \\
\hline FY 98 & & $244 \mathrm{TXR}$ & WASTE DISPOSAL VALLT, L'NDERGROLNDVALLT & LMHC & TX-FARM & & & & $\mathrm{PCx}$ & & & \\
\hline FY 98 & & 2440 & $\begin{array}{l}\text { SALT WELL RECENVER VAULTRECEIVER } \\
\text { TANKU-FARM DCRT }\end{array}$ & LMHC & U-FARM & & & 16 & $\mathrm{PCx}$ & & & \\
\hline FY 98 & & $244 \mathrm{U1}$ & INSTRLMENT BLTINING & LMHC & & & & . & $\mathrm{PCx}$ & & & \\
\hline FY 98 & & $245 \mathrm{~A}$ & PDD $/ A S D$ LIFT STATION NO. 1 & PHMC & & & & & $\mathrm{PCx}$ & & & \\
\hline FY 98 & & $251 \mathrm{AX} 801$ & WATER SERVICE BULDING & LMHC & & & & & $\mathbf{P C x}$ & & & \\
\hline FY 98 & & $251 \mathrm{~W}$ & $\begin{array}{l}\text { PRIMARY } 230 \text { VV SWITCHING STATION,NO. OF } \\
\text { 20OWELECTRICAL SLBSTATION }\end{array}$ & PHMC & & & & 388 & $\mathrm{PCx}$ & & & \\
\hline
\end{tabular}




\begin{tabular}{|c|c|c|c|c|c|c|c|c|c|c|c|c|}
\hline $\begin{array}{l}\text { EvALU } \\
\text { SCHED }\end{array}$ & $\begin{array}{l}\text { PKLIOITY } \\
\text { SCORE }\end{array}$ & IDEXTIFIER & DESCRIPTION & CONTRA & PROJECT & $\begin{array}{l}\text { YEAR } \\
\text { BLTITT }\end{array}$ & $\begin{array}{l}\text { YEAR } \\
\text { SURPLES }\end{array}$ & $\begin{array}{l}\text { AREA SQ* } \\
\text { METERS }\end{array}$ & $\begin{array}{l}\text { PERF.C } \\
\text { AT. }\end{array}$ & $\begin{array}{l}\text { TOTAL } \\
\text { OCCLP }\end{array}$ & $\begin{array}{l}\text { FACILITY } \\
\text { CONDITION } \\
\text { RATING }\end{array}$ & $\begin{array}{l}\text { EXISTING } \\
\text { ANALYSIS } \\
\text { RATING }\end{array}$ \\
\hline FY 98 & & $252 \mathrm{BY}$ & SLBSTATION $13.8 \mathrm{kV}$ & LMHC & & & & & PCx & & & \\
\hline FY 98 & & $252 \mathrm{E}$ & $\begin{array}{l}\text { ELECTRICAL. SWITCHING } \\
\text { STATION13.\&KVELECTRICAL SLBSTATION }\end{array}$ & PHMC & & & & 34 & $\mathrm{PCx}$ & & & \\
\hline FY 98 & & $252 \mathrm{U}$ & $\begin{array}{l}\text { 13.8 KV SLEBSTATTON, ON CONCRETESLAB } \\
\text { ELECTRICAL CBSS SUESTATION }\end{array}$ & PHMC & & & & & $\mathrm{PCx}$ & & & \\
\hline FY 58 & & $252 \mathrm{~W}$ & $\begin{array}{l}\text { ELECTRICAL SWITCHING STATION } \\
\text { 13.8KVELECTRFCAL SUBSTATION }\end{array}$ & PHMC & T-PLANT & & & 34 & $\mathrm{PCX}$ & & & \\
\hline FY 58 & & 261409 & $\begin{array}{l}\text { ENVIRONMENTAL } \\
\text { MONITORINGSTATIONENVIRONMENTAL } \\
\text { MONITORINGSTATION }\end{array}$ & PHMC & & & & 5 & $\mathrm{PCx}$ & & & \\
\hline FY 98 & & $2614 \mathrm{WD}$ & MONITORING STATION & PHMC & & & & & $\mathrm{PCx}$ & & & \\
\hline$F Y 98$ & & $267 \mathrm{Z}$ & RISER \# VALVE HOUSE & PHMC & PFP & & & 4 & $\mathrm{PCx}$ & & & \\
\hline $\mathrm{FY} 98$ & & $2701 \mathrm{AC}$ & PUREX PATROL GUARD SHACKPUREX PARTROL & PHMC & PLREX & & & 2 & $\mathrm{PCx}$ & & & \\
\hline FY 98 & & $2703 \mathrm{E}$ & CHEMICAL ENGINEERRIG LABORATORY & LMHC & & & & 354 & $\mathrm{PCX}$ & & & \\
\hline $\mathrm{FY} 98$ & & $2707 \mathrm{AR}$ & SLUDGE VAULT CHANGE HOUSE & LMHC & & & & 40 & $\mathrm{PCX}$ & & & \\
\hline FY 98 & & $2707 \mathrm{AX}$ & BUILDING CHANGE & LMHC & & & & 30 & $\mathrm{PCx}$ & & & \\
\hline $\mathrm{FY} 98$ & & $27075 \mathrm{X}$ & CHANGE HOUSE & LMHC & & & & 132 & $\mathrm{PCx}$ & & & \\
\hline FY 98 & & 2708AR & SOILED LAUSDRY STORAGE BUILDING & LMHC & PLREX & & & 7 & $\mathrm{PCx}$ & & & \\
\hline FY 98 & & $270 \mathrm{~W}$ & $\begin{array}{l}\text { UNDERRGROUND CONDENSATENELTRALZATION } \\
\text { TANKNEUTRALIZATION TANK }\end{array}$ & LMHC & & & & & $\mathrm{PCx}$ & & & \\
\hline FY 98 & & $2712 \mathrm{~B}$ & $\begin{array}{l}\text { ELECTRLAL } \\
\text { LNSTRUMENTATIONBULDINGMONITORING } \\
\text { BUILDING }\end{array}$ & PHMC & B-PLANT & & & 2 & $\mathrm{PCX}$ & & & \\
\hline$F Y 98$ & & $2712 \mathrm{C}^{\circ}$ & ELECTRICAL INSTRUMENTATTON BUILDING & RFSH & & & & & PCy & & & \\
\hline FY 98 & & $2733 \mathrm{~W}$ & GARAGE OFFICE \& SERVICE & PHMC & & & & 387 & $\mathrm{PCx}$ & & & \\
\hline FY 98 & & $2713 \mathrm{WB}$ & $\begin{array}{l}\text { REGULATED GARAGE AND HEAVY EQUTPMENT } \\
\text { REPAIR }\end{array}$ & LMAC & & & & 572 & $\mathrm{PCx}$ & & & \\
\hline FY 98 & & 2713wC & PESTICIDE WASHWATER RECOVERYFACILITY & PHMC & & & & 163 & $\mathrm{PCx}$ & & & \\
\hline FY 98 & & 2714AR & SLUDGE VAULT GENERAL STORAGE BUILDING & LMHC & & & & 7 & $\mathrm{PCX}$ & & & \\
\hline FY 98 & & $2714 \mathrm{U}$ & WAREHOUSE U0 3 STORAGE BUILDING & PHMC & Lo3 & & & 401 & $\mathbf{P C x}$ & & & \\
\hline FY 98 & & 2715AW & STORAGE AND STAGLG BUILDLNG & LMHC & & & & & $\mathrm{PCx}$ & & & \\
\hline $\mathrm{FY} 9 \mathrm{~B}$ & & 2715EA & DRUM STORAGE SHEDDRUM STORAGE SHED & PHMC & & & & 18 & $\mathrm{PCX}$ & & & \\
\hline FY 98 & & $2716 \mathrm{~A}$ & RM CHECK OUT \$TATION, NEARTUNNEL & PHMC & PLREX & & & . & $\mathrm{PCx}$ & & & \\
\hline FY 98 & & $2716 \mathrm{E}$ & $\begin{array}{l}\text { POWER MAINTENANCE STORAGEBUIDLDNGPOWER } \\
\text { MAINTENANACE STORAGEBULDING }\end{array}$ & PHMC & & & & 73 & $\mathrm{PCx}$ & & & \\
\hline FY 98 & & $271 \mathrm{~A}$ & INSTRLMENT BLDG & PHMC & & & & & $\mathrm{PCx}$ & & & \\
\hline FY 98 & & $271 \mathrm{AN}$ & INSTRUMENT RLDG & PHMC & & & & & $\mathrm{PCx}$ & & & \\
\hline FY 98 & & 271AP & INSTRLMENT CONTROL BLDG & PHMC & & & & & $\mathrm{PCx}$ & & & \\
\hline
\end{tabular}




\begin{tabular}{|c|c|c|c|c|c|c|c|c|c|c|c|c|}
\hline $\begin{array}{l}\text { EVALU } \\
\text { SCHED }\end{array}$ & $\begin{array}{l}\text { PRIOITY } \\
\text { SCORE }\end{array}$ & IDENTTFIER & DESCRIYTION & CONTRA & PROJECT & $\begin{array}{l}\text { YEAR } \\
\text { BEILT }\end{array}$ & $\begin{array}{l}\text { YEAR } \\
\text { SURPLUS }\end{array}$ & $\begin{array}{l}\text { AKEA SQ\# } \\
\text { METERS }\end{array}$ & $\begin{array}{c}\text { PERF.C } \\
\text { AT. }\end{array}$ & $\begin{array}{l}\text { TOTAI } \\
\text { OCCLP }\end{array}$ & $\begin{array}{l}\text { FACILITY } \\
\text { CONDITION } \\
\text { RATING }\end{array}$ & $\begin{array}{l}\text { EXISTING } \\
\text { ANALYSIS } \\
\text { RATING }\end{array}$ \\
\hline FY 98 & & $271 \mathrm{AW}$ & LNSTRUMENT BLDG & PHMC & & & & & $\mathrm{PCx}$ & & & \\
\hline FY 98 & & $27 \mathrm{CR}$ & SER VICE AND OFFICE BUILIDNG & LMHC & PUREX & & & 125 & $\mathrm{PCx}$ & & & \\
\hline FY 98 & & $2724 \mathrm{~A}$ & $\begin{array}{l}\text { RADIATION MONTTORING AND PROTECTIVE } \\
\text { CLOTHING FAC. }\end{array}$ & LMHC & & & & & $\mathrm{PCx}$ & & & \\
\hline $\mathrm{FY} 98$ & & $2724 \mathrm{AY}$ & $\begin{array}{l}\text { RADIATION MONITORING AND PROTECTIVE } \\
\text { CLOTHING FAC }\end{array}$ & LMHC & & & & 6 & $\mathrm{PCx}$ & & & \\
\hline FY 98 & & $2724 A Z$ & CHANGE HOUSE, TANK FARMS & LMHC & & & & 6 & $\mathrm{PCx}$ & & & \\
\hline FY 98 & & $2724 \mathrm{~B}$ & $\begin{array}{l}\text { RAD. MONITORTEG AND PROTECTIVE CLOTHING } \\
\text { FACILITY }\end{array}$ & T.MHC & TWRS & & & 6 & $\mathrm{PCx}$ & & & \\
\hline FY 98 & & $2724 \mathrm{BX}$ & $\begin{array}{l}\text { RAD. MONITORING AND PROTECTVE CLOTHING } \\
\text { FACILITY }\end{array}$ & LMHC & & & & 6 & $P C x$ & & & \\
\hline FY 98 & & $2724 B Y$ & BUILDLNG CHECKOLT & I.MHC & & & & 6 & PCx & & & \\
\hline FY 98 & & $2724 \mathrm{C}$ & $\begin{array}{l}\text { RADIATION MONITORING AND PROTFCTTVE } \\
\text { CLOTHING FAC. }\end{array}$ & LMHC & C.Fami & & & 6 & $\mathrm{PCX}$ & & & \\
\hline FY 98 & & $2724 C A$ & $\begin{array}{l}\text { RADIATION MONITORING AND PROTECTIVE } \\
\text { CLOTHING FAC. }\end{array}$ & LMHC & C.FattrL & & & 6 & $\mathrm{PC}$ & & & \\
\hline FY 98 & & $2724 \mathrm{SX}$ & $\begin{array}{l}\text { RADLATION MONTTORLNG AND PROTECIIVE } \\
\text { CLOTHING FAC. }\end{array}$ & L.MHC & & & & 6 & $\mathrm{PCX}$ & & & \\
\hline FY 98 & & $27245 Y$ & $\begin{array}{l}\text { RADIATION MONTTORING AND PROTECTIVE } \\
\text { CLOTHING FAC. }\end{array}$ & LMHC & & & & 6 & $\mathrm{PCx}$ & & & \\
\hline FY 98 & & $2724 \mathrm{~T}$ & $\begin{array}{l}\text { RADIATION MONITORLEG AND PROTECTTVE } \\
\text { CLOTHING FAC. }\end{array}$ & LMHC & & & & 6 & $\mathrm{PCx}$ & & & \\
\hline FY 98 & & $2724 \mathrm{KX}$ & $\begin{array}{l}\text { RADLATION MONITORING AND PROTECTIVE } \\
\text { CLOTHING FAC. }\end{array}$ & LMHC & & & & 6 & $\mathrm{PCx}$ & & & \\
\hline FY 98 & & $2724 \mathrm{TXA}$ & $\begin{array}{l}\text { RADIATION MONITORNGG AND PROTECTIVE } \\
\text { CLOTHING FAC. }\end{array}$ & LMHC & & & & 6 & $\mathrm{PCx}$ & & & \\
\hline FY 98 & & $2724 \mathrm{TXB}$ & $\begin{array}{l}\text { RADIATION MONTTORING AND PROTECTNE } \\
\text { CLOTHLNG FAC. }\end{array}$ & L.MHC & & & & 6 & $\mathrm{PCx}$ & & & \\
\hline $\mathrm{HY} 98$ & & $2724 \mathrm{U}$ & CHECKOUT BULDING & LMHC & & & & 6 & $\mathbf{P C x}$ & & & \\
\hline FY 98 & & $2724 \mathrm{LA}$ & $\begin{array}{l}\text { RADLATON MONITORING AVDPROTECTIVE } \\
\text { CLOTHNG FAC }\end{array}$ & LMHC & & & & & $\mathrm{PCX}$ & & & \\
\hline FY 98 & & $272 \mathrm{TWA}$ & SODIUM STORAGE BUTLDING & LMHC & & & & 248 & $\mathrm{PCX}$ & & & \\
\hline$F Y \$$ & & $272 \mathrm{~A}$ & MALVTENANCE STORAGE & LMHC & PLREX & & & 44 & $\mathrm{PCX}$ & & & \\
\hline FY 98 & & $272 A W$ & TANK FARM OPERATIONS SLIPPORT FACILTYY & LMHC & & & & 1356 & $\mathrm{PCx}$ & & & \\
\hline FY 98 & & $272 \mathrm{AW} 10$ & $\begin{array}{l}\text { CALIBRATION SOURCE } \\
\text { STORAGEBLILDNGCALIBRATION SOLRCE } \\
\text { STORAGEBUILDING }\end{array}$ & LMHC & & & & & $\mathrm{PCx}$ & & & \\
\hline FY 98 & & $272 \mathrm{BC}$ & $\begin{array}{l}\text { CONSTRUCTION MULTICRAFT } \\
\text { STORAGECONSTRLCTION STORAGE BUILDENG }\end{array}$ & PHMC & & & & 232 & $\mathrm{PCx}$ & & & \\
\hline FY 98 & & $272 \mathrm{EA}$ & SWP CHANGE SHELTER & PHMC & & & & 4 & $\mathbf{P C x}$ & & & \\
\hline FY 98 & & 2725 & MANTENANCE SHOP MACHINE SHOP & LMHC & & & & 421 & $\mathbf{P C X}$ & & & \\
\hline
\end{tabular}




\begin{tabular}{|c|c|c|c|c|c|c|c|c|c|c|c|c|}
\hline $\begin{array}{l}\text { EVALU } \\
\text { SCHED }\end{array}$ & $\begin{array}{l}\text { PRIOITY } \\
\text { SCORE }\end{array}$ & IDENTIFIER & DESCRIPTION & CONTRA & pROJECT & $\begin{array}{l}\text { YEAR } \\
\text { BUILT }\end{array}$ & $\begin{array}{l}\text { YEAR } \\
\text { SCERPLUS }\end{array}$ & $\begin{array}{l}\text { AREA SQH } \\
\text { METERS }\end{array}$ & $\begin{array}{c}\text { PERF.C } \\
\text { AT. }\end{array}$ & $\begin{array}{l}\text { TOTAL, } \\
\text { OCCUP }\end{array}$ & $\begin{array}{l}\text { FACILITY } \\
\text { CONDITION } \\
\text { RATING }\end{array}$ & $\begin{array}{l}\text { EXISTING } \\
\text { ANALYSIS } \\
\text { RATING }\end{array}$ \\
\hline FY 98 & & $272 \mathrm{WA}$ & $\begin{array}{l}\text { TANK FARM SUPPORT FACLLITY TANK FARM } \\
\text { OPER } 200-W\end{array}$ & LMHC & & & & 1738 & $\mathrm{PC} x$ & & & \\
\hline FY 98 & & $2734 \mathrm{EA}$ & GAS CYLINDER STORAGE BULDING & PHMC & & & & & $\mathrm{PCx}$ & & & \\
\hline FY 98 & & $273 \mathrm{AN}$ & SERYICE BLDG & PHMC & & & & & $\mathrm{PCx}$ & & & \\
\hline FY 98 & & $273 \mathrm{AP}$ & COMPRESSOR BI.DG & PHMC & & & & & $\mathrm{PCx}$ & & & \\
\hline FY 98 & & $273 \mathrm{AW}$ & SERVTCE BLDG & PHMC & & & & & $\mathrm{PCX}$ & & & \\
\hline FY 98 & & $273 \mathrm{EA}$ & STORAGE YARD & LMHC & & & & & $\mathrm{PCX}$ & & & \\
\hline FY 98 & & $274 \mathrm{AZ}$ & $\begin{array}{l}\text { RADLATION MONITORING AND PROTECTIVE } \\
\text { CLOTHING FAC. }\end{array}$ & PHMC & & & & & $\mathrm{PCx}$ & & & \\
\hline FY 98 & & 2802AR & SLLDGE VALILT STEAM DISTRLBU TONSYSTEM & LMHC & & & & & $\mathrm{PCx}$ & & & \\
\hline FY 98 & & $2803 \mathrm{AR}$ & COMPRESSED AIR DISTRRBUTIONSYSTEM & LMHC & & & & & $\mathrm{PCX}$ & & & \\
\hline FY 98 & & $282 \cdot \mathrm{ED}$ & STANDBY GENERATOR & PHMC & & & & & $\mathrm{PCx}$ & & & \\
\hline FY 98 & & $282 \mathrm{WD}$ & STANDBY GENERATOR & PHMC & & & & & $\mathrm{PCX}$ & & & \\
\hline FY 98 & & $283 \mathrm{WB}$ & EQUALTZATTON BASIN \& PUMP STATION & PHMC & & & & & $\mathrm{PCx}$ & & & \\
\hline FY 98 & & $284 \mathrm{~EB}$ & POWER HOLISE FILTRATION BULDDING & PHMC & & & & 367 & $\mathrm{PCx}$ & & & \\
\hline FY 98 & & $285 \mathrm{w}$ & BACKFLOW PREVENTION BUILONG & PHMC & $\mathrm{CWC}$ & & & 9 & $\mathrm{PCx}$ & & & \\
\hline FY 98 & & $2901 \mathrm{AR}$ & SLLDGE VAULT WATER STORAGEPIPING & LMHC & & & & & $\mathrm{PC} x$ & & & \\
\hline FY 98 & & $2 \times 04 \mathrm{AR}$ & SLUDGE VALLT PROCESS SEWERSYSTEM & LMHC & & & & & $\mathrm{PCx}$ & & & \\
\hline FY 98 & & $291 \mathrm{AD}$ & $\begin{array}{l}\text { FILTER PIT AND STACKEXHAUST FILTER } \\
\text { BUILDNG }\end{array}$ & PHMC & PLiREX & & & 17 & $\mathrm{PCX}$ & & & \\
\hline FY 98 & & $291 \mathrm{AH}$ & AOG SAMPLE STATION & PHMC & PUREX & & & 9 & $\overline{P C x}$ & & & \\
\hline FY 98 & & $291 \mathrm{CR}$ & RECOVERY FACILITIES IN 24IC TANK FARM STACK & LMHC & & & & . & PCx & & & \\
\hline FY 98 & & $292 \mathrm{AR}$ & $\begin{array}{l}\text { FLLTER PIT AND INSTRUMENT HOUSE VESSEL } \\
\text { VENT STACK }\end{array}$ & LMHC & & & & 7 & $\mathrm{PCX}$ & & & \\
\hline FY 98 & & $295 \mathrm{~A} 1$ & CRIB SAMPLER HOUSE & PHMC & & & & & $\mathrm{PCx}$ & & & \\
\hline$F Y 98$ & & $295 \mathrm{~A} 2$ & SAMPLE STATION & PHMC & & & & 4 & PCx & & & \\
\hline FY 98 & & $296 \mathrm{Al2}$ & STACK, 244AR VAULT VESSEL VEATILATION & LMHC & & & & & $\mathrm{PCx}$ & & & \\
\hline FY 98 & & $295 \mathrm{~A} 13$ & $\begin{array}{l}\text { STACK, 244AR VAULT CANYONCELLS } \\
\text { VENTILATION }\end{array}$ & LMHC & & & & & $\mathrm{PCx}$ & & & \\
\hline$F Y 98$ & & $296 \mathrm{A17}$ & STACK, 241AY/AZ TK FM PRLM TK VENTLATION & LMFC & & & & & $\mathrm{PCx}$ & & & \\
\hline Fy go & & $296 \mathrm{Al}$ & STACK, 24IAY10I TANK ANNULLS VENTILATION & LMHC & & & & & $\mathrm{PCx}$ & & & \\
\hline FY 98 & & $295 \mathrm{~A} 19$ & STACK, 241AYI02 TANK ANNLLUS VENTILATION & LMHC & & & & & PCx & & & \\
\hline FY 98 & & $296 \mathrm{~A} 20$ & STACK, 241AZ TANK ANNULI EXHAUST & LMHC & & & & & $\mathrm{PCx}$ & & & \\
\hline $\mathrm{FY} 98$ & & $296 \mathrm{~A} 25$ & $\begin{array}{l}\text { STACK, 244A DC REC:R TK/ANNELLLS } \\
\text { VENTILATION }\end{array}$ & LMHC & & & & & $\mathrm{PCX}$ & & & \\
\hline $\mathrm{FY} 98$ & & $295 \mathrm{~A} 26$ & STACK, 204AR UNLOAD RM/SIMP TK VENT & LMHC & & & & & $\mathrm{PCx}$ & & & \\
\hline $\mathrm{FY} 98$ & & $296 \mathrm{~A} 27$ & AW TANK FARM PRIM TANK VENTILATION & LMHC & & & & & $\mathrm{PCX}$ & & & \\
\hline
\end{tabular}




\begin{tabular}{|c|c|c|c|c|c|c|c|c|c|c|c|c|}
\hline $\begin{array}{l}\text { EVALU } \\
\text { SCHED }\end{array}$ & $\begin{array}{l}\text { PRIOFTY } \\
\text { SCORE }\end{array}$ & IDENTIFIER & DESCRIPTION & $\operatorname{CONTRA}$ & PROIECT & $\begin{array}{l}\text { YEAR } \\
\text { BUILT }\end{array}$ & $\begin{array}{c}\text { YEAR } \\
\text { SURPLUS }\end{array}$ & $\begin{array}{l}\text { AREA SQ\# } \\
\text { METERS }\end{array}$ & $\begin{array}{c}\text { FERT.C } \\
\text { AT. }\end{array}$ & $\begin{array}{l}\text { TOTAL } \\
\text { OCCUP }\end{array}$ & $\begin{array}{l}\text { FACILITY } \\
\text { CONDITION } \\
\text { RATING }\end{array}$ & $\begin{array}{l}\text { EXISTING } \\
\text { ANALYSIS } \\
\text { RATING }\end{array}$ \\
\hline FY 98 & & $296 \mathrm{~A} 28$ & STACK, AW TANK FM ANNLIUS VENTILATON & LMHC & & & & & $\mathrm{PCx}$ & & & \\
\hline FY 98 & & $296 \mathrm{~A} 29$ & $\begin{array}{l}\text { STACK, AN TANK FARM PRIMARY TANK } \\
\text { VENTILATION }\end{array}$ & LMHC & & & & & $\mathbf{P C x}$ & & & \\
\hline FY 98 & & 296430 & STACK. AN TANK FARM ANNLLUS VENTILATTON & LMHC & & & & & $P C x$ & & & \\
\hline $\mathrm{FY} 98$ & & $296 \mathrm{~A} 40$ & STACK, AP TK FM PRLMARY TANK VENTILATION & LMHC & & & & & $\mathrm{PCx}$ & & & \\
\hline FY 98 & & $296 \AA 41$ & STACK, AP TANK FARM ANNLZITS VENTILAIION & LMHC & & & & & $\mathbf{P C x}$ & & & \\
\hline FY 98 & & 296442 & AGING WASTE TANK STACK BULT PROJECT W03 & LMHC & & & & & $\mathrm{PCX}$ & & & \\
\hline FY 98 & & $296 \mathrm{~A} 43$ & AGING WASTE BLILDLNG STACK (W030) & LMHC & & & & & $\mathrm{PCx}$ & & & \\
\hline FY 98 & & $206 \mathrm{~B} 28$ & STACK, 244BX DCRT A ANNULLS VENTLATION & LMHC & & & & & $\mathrm{PCx}$ & & & \\
\hline FY 98 & & $296 C 5$ & STACK, 24ACR VAULT AND CELL VENTILATION & LMFIC & & & & & $\mathrm{PCx}$ & & & \\
\hline FY 98 & & $296 \mathrm{C6}$ & 106-C STACK ENDER CONSTRUCTON (W330) & LMHC & & & & & $\mathrm{PCx}$ & & & \\
\hline FY 98 & & $296 \mathrm{C7}$ & 103-C VAPOR MIXER STACK & LMHC & & & & & $\mathrm{PCx}$ & & & \\
\hline FY 98 & & 296001 & GROUT EXHAUSTER & LMHC & & & & & $\mathrm{PCx}$ & & & \\
\hline FY 98 & & $296 \mathrm{P} 16$ & $\begin{array}{l}\text { POR TABLE EXHALISTER FOR TANK } \\
\text { S24IC104/105:106 }\end{array}$ & LMHC: & C-Farm & & & & $\mathrm{PCx}$ & & & \\
\hline FY 98 & & $296 \mathrm{P} 17$ & $\begin{array}{l}\text { PORTABLE EXHALSTER FOR TANK } \\
\text { S241A104/105/106 } \\
\end{array}$ & LMHC & A. Farm & & & & $P C x$ & & & \\
\hline FY 98 & & $296 \mathrm{P} 22$ & $\begin{array}{l}\text { STACK, 24ISY TANK FARM ANNULUS } \\
\text { VENTLATTON }\end{array}$ & LMHC & & & & & $\mathrm{PCx}$ & & & \\
\hline FY 98 & & $296 \mathrm{P} 23$ & $\begin{array}{l}\text { STACK, } 2415 Y \text { TARK FARM PRIMARY TANK } \\
\text { VENTILATION }\end{array}$ & LMHC & & & & & $\mathrm{PCX}$ & & & \\
\hline FY 98 & & $296 \mathrm{P26}$ & $\begin{array}{l}\text { STACK, 241AY AZ TF PRIM TK BACKUIP VENT, } \\
296 \text { A17 }\end{array}$ & LMHC & & & & & $\mathrm{PCx}$ & & & \\
\hline FY 98 & & $296 \mathrm{P28}$ & $\begin{array}{l}\text { STACK, 24ISY TK FM PRIM TK BACKUP VENT, } \\
\text { 296P23 }\end{array}$ & LMHC & & & & & $\mathrm{P} C x$ & & & \\
\hline FY 98 & & $296 \mathrm{P} 20$ & NEXT TO 244.S & LMHC & & & & & $\mathrm{PCx}$ & & & \\
\hline$F Y 98$ & & $296 \mathbf{P 3 1}$ & $\begin{array}{l}\text { STACK, 209E CRITICAL MASS LABORATORY } \\
\text { VENTILATON }\end{array}$ & LMHC & & & & & PCx & & & \\
\hline FY 98 & & $296 \mathbf{P} 40$ & $\begin{array}{l}\text { TANK FARM PORTABLE EXHAUSTER } \propto 209-\mathrm{E} \\
\text { GREEN HOUSE200 EAST }\end{array}$ & LMEC & & & & & $\mathrm{PCX}$ & & & \\
\hline FY 98 & & $296 \$ 15$ & STACK, $2415 \times 107-112 \& 241 \mathrm{SX} 114$ VENTLATION & LMHC & $S X-F A R M$ & & & & $\mathrm{PCx}$ & & & \\
\hline FY 98 & & $296 \$ 18$ & STACK, $242 S$ EVAPORATOR BLDG VENTILATION & LMHC & & & & & $\mathrm{PCx}$ & & & \\
\hline FY 98 & & $296 \$ 22$ & STACK, $244 S$ DCRT \& ANNULUS VENTILATION & LMHC & & & & & $\mathrm{PCx}$ & & & \\
\hline FY 98 & & 296525 & 241-SY TANK FARM REPLACEMENT EXHAUSTER & LMHC & & & & & $\mathrm{PCx}$ & & & \\
\hline FY 98 & & $296 \mathrm{T17}$ & $\begin{array}{l}\text { STACK, 242T EVAP FAC \& COLD SIDE } \\
\text { VENTILATON }\end{array}$ & LMHC & & & & & $\mathrm{PCx}$ & & & \\
\hline FY 98 & & $296 \mathrm{~T} 18$ & STACK. 244TX SALT WELL RECERVR EXHALST & LMHC & & & & & $\mathbf{P C x}$ & & & \\
\hline FY 98 & & 296011 & STACK, 244U DCRT \& ANNULLS VENTILATION & LMHC & & & & & $\mathrm{PCx}$ & & & \\
\hline
\end{tabular}




\begin{tabular}{|c|c|c|c|c|c|c|c|c|c|c|c|c|}
\hline $\begin{array}{l}\text { EVALC: } \\
\text { SCHED }\end{array}$ & $\begin{array}{l}\text { PRIOITY } \\
\text { SCORE }\end{array}$ & WENTIFIER & DESCRIPTION & CONTRA & PROJECT & $\begin{array}{l}\text { YEAR } \\
\text { BUILT }\end{array}$ & $\begin{array}{c}\text { YEAR } \\
\text { St:RPLUS }\end{array}$ & $\begin{array}{l}\text { AREA SQ } \\
\text { METERS }\end{array}$ & $\begin{array}{c}\text { PERF.C } \\
\text { AT. }\end{array}$ & $\begin{array}{l}\text { TOTAL } \\
\text { OCCUP }\end{array}$ & $\begin{array}{l}\text { FACILITY } \\
\text { CONDITION } \\
\text { RATING }\end{array}$ & $\begin{array}{l}\text { EXISTING } \\
\text { ANALYSIS } \\
\text { RATING }\end{array}$ \\
\hline FY 98 & & $296 \mathrm{~W} 03$ & 213-W WASTE COMPACTOR EXHAUIST & LMHC & & & & & $\mathrm{PCx}$ & & & \\
\hline FY 98 & & 301WELLSIAV & STORAGE BULDINGSTORAGE & PHMC & & & & 929 & $\mathrm{PCx}$ & & & \\
\hline FY 98 & & $303 \mathrm{P} 41$ & PROCESS EFFLUENT TREATMENTFACILITY & PHMC & & & & & $\mathrm{PCx}$ & & & \\
\hline FY 98 & & $305 \mathrm{~A}$ & ELECTRICIAN \& PIPEFTTER SHOP & PHMC & & 1948 & & 274 & $\mathrm{PCx}$ & 0 & & \\
\hline FY 98 & & 3060 CWWWHS3 & HANFORD SQUARE ШI OFFICE BUILDING & DYN & & & & & $\mathbf{P C Y}$ & & & \\
\hline FY 98 & & 3070 GWWHS2 & HANFORD SQUARE IIOFFICE BUILDNG & PHMC & & & & 1338 & $\mathrm{PCX}$ & & & \\
\hline FY 98 & & 3080GWWHS1 & HANFORD SQUARE I OFFICE BUILDING & DYN & & & & & $\mathrm{PCy}$ & & & \\
\hline FY 98 & & 3090GWWHS4 & HANFORD SQUARE NOFFICE BUILDING & PHMC & & & & 1070 & $\mathrm{PCx}$ & & & \\
\hline $\mathrm{FY} 98$ & & $3100 \mathrm{GWW}$ & $\begin{array}{l}\text { 3100 GEO. WASH. WAY (RICHLANDIOFFCE, } \\
\text { BLILDLNG }\end{array}$ & PHMC & & & & 879 & $\overline{\mathrm{PC} x}$ & & & \\
\hline $\mathrm{FY} 98$ & & $310 \mathrm{~S}$ & DRUM STORAGE AREA - TEDF & PHMC & & & & & $\mathrm{PCx}$ & & & \\
\hline FY 98 & & 313 & REACTOR FUEL MAVLFACTURLVG BUILOING & PHMC & N-Fuels & & & 7133 & $\mathrm{PCx}$ & & & \\
\hline FY 98 & & 317 & CALIBRATION AND STANDARDSFACILITY & PHMC & & & & & $\mathrm{POX}$ & & & \\
\hline FY 98 & & $321 \mathrm{~B}$ & $\begin{array}{l}\text { MODEL HEAT LOOP, PART OF } 32 \text { IBULDINGMODEL } \\
\text { TEST LOOP }\end{array}$ & PHMC & & & & 161 & $\mathrm{PCx}$ & 0 & & \\
\hline FY 98 & & $321 \mathrm{D}$ & SEISMIC TESTING FAC. PART OF 321RLDG & PHMC & & & & & $\mathrm{PCx}$ & & & \\
\hline FY 98 & & 331 IWCLEAR & $\begin{array}{l}\text { 3311 W. CLEARWATER, KENNEWICKOFFCE - } \\
\text { LEASED }\end{array}$ & PHMC & & & & 342 & $\mathrm{PCX}$ & & & \\
\hline FY 98 & & 3408 & FMT COOLNG TOWER & PHMC & & & & & PCx & & & \\
\hline FY 98 & & $351 \mathrm{~A}$ & $\begin{array}{l}\text { METER AND TESTING BUTLDINGELECTRICAL } \\
\text { SUBSTATION }\end{array}$ & PHMC & & 1952 & & 26 & $\mathrm{PCx}$ & 0 & & \\
\hline FY 98 & & $351 \mathrm{~B}$ & $\begin{array}{l}\text { METER TESTING AND SWITCHGEARFACILTTY, } \\
\text { B3S4ELECTRICAL SLBSTATION }\end{array}$ & PHMC & N-Fuels & 1970 & & 149 & $\mathrm{PCx}$ & 0 & & \\
\hline FY 98 & & $352 \mathrm{E}$ & $\begin{array}{l}\text { SWITCH STATFN EAST SIDEELECTRICAL } \\
\text { SUBSTATION — EAST SWE }\end{array}$ & PHMC & & 1972 & & 149 & $\mathrm{PCX}$ & 0 & & \\
\hline FY 98 & & $352 \mathrm{~F}$ & $\begin{array}{l}\text { ELECTRICAL SLBSTATION } 2,4 \text { KVELECTRICAL } \\
\text { SLRSTATION }\end{array}$ & PHMC & & 1978 & & 56 & $\mathbf{P C x}$ & 0 & & \\
\hline FY 58 & & 360 & SANITARY SEWER CONTROL BULDING & PHMC & & & & 173 & $\mathbf{P C x}$ & & & \\
\hline FY 98 & & $3607 \mathrm{~A}$ & PUMPHOUSEPUMPHOUSE & PHMC & & & & 9 & $\mathbf{P C x}$ & 0 & & \\
\hline FY 98 & & $3621 \mathrm{E}$ & INLNTERRLPTIBLE POWER SLPPLYBULUING & PHMC & & & & & PCx & & & \\
\hline FY 98 & & $3701 \mathrm{U}$ & OFFICE BULDDINGFAURD STATIN & PHMC & & 1579 & & 125 & $\mathrm{PCx}$ & 0 & & \\
\hline FY 98 & & 3704 & INSULATORS STORAGE STORAGE BUILDING & PHMC & N.Fuels & 1944 & & 74 & $\mathrm{PCx}$ & 0 & & \\
\hline FY 98 & & 3705 & $\begin{array}{l}\text { PHOTOGRAPHY BULLDINGPHOTOGRAPHY } \\
\text { BUILDING }\end{array}$ & PHMC & N-Fuels & 1944 & & 648 & $\mathrm{PCx}$ & 15 & & \\
\hline FY 98 & & $3706 \mathrm{~A}$ & $\begin{array}{l}\text { VENTHATION EQUTP RM FOR } \\
\text { 3706BUHLDING VENTILATION FOR } 3706\end{array}$ & PHMC & & 1944 & & 140 & $\mathrm{PCX}$ & 0 & & \\
\hline FY 98 & & $3707 \mathrm{E}$ & CONSTRUCTION STORAGE STORAGE BULLING & PHMC & & 1944 & & 74 & $\mathrm{PCx}$ & 0 & & \\
\hline FY 98 & & $3707 \mathrm{H}$ & CHANGE HOUSECHANGE HOUSE & PHMC & & 1979 & & 112 & $\mathrm{PCx}$ & 0 & & \\
\hline
\end{tabular}




\begin{tabular}{|c|c|c|c|c|c|c|c|c|c|c|c|c|}
\hline $\begin{array}{l}\text { EVALE: } \\
\text { SCHED }\end{array}$ & $\begin{array}{l}\text { PRIOITY } \\
\text { SCORE }\end{array}$ & IDENTIFIER & DESCRIPTION & CONTRA & PROJECT & $\begin{array}{l}\text { YEAR } \\
\text { BUILT }\end{array}$ & $\begin{array}{l}\text { YEAR } \\
\text { SURPLUS }\end{array}$ & $\begin{array}{l}\text { AREA SQ⿻ } \\
\text { METERS }\end{array}$ & $\begin{array}{l}\text { PERF.C } \\
\text { AT. }\end{array}$ & $\begin{array}{l}\text { TOTAL } \\
\text { OCCUP }\end{array}$ & $\begin{array}{l}\text { FACILITY } \\
\text { CONDITION } \\
\text { RATING }\end{array}$ & $\begin{array}{l}\text { EXISTING } \\
\text { ANALYSIS } \\
\text { RATING }\end{array}$ \\
\hline FY 98 & & 3714 & ORGANIC CHEMISTRY LABORATORY & PHMC & & 1955 & & 107 & $\mathrm{PCx}$ & 0 & & \\
\hline FY 98 & & 3717 & $\begin{array}{l}\text { SPARE PARTS WAREHOUSE WARFHOLISE } \\
\text { BIILDING }\end{array}$ & PHMC & $341-\mathrm{WHF}$ & 1944 & & 897 & $\overline{\mathrm{PCx}}$ & 0 & & \\
\hline FY 98 & & $3718 \mathrm{C}$ & STORAGE BUILUINGSTORAGE BUILDING & PHMC & & 1952 & & 416 & $\mathrm{PCX}$ & 0 & & \\
\hline FY 98 & & $3718 \mathrm{~N}$ & INSULATION SHOPELECTRICAL SHOP & PHMC & & 1971 & & 268 & $\mathrm{PCx}$ & 2 & & \\
\hline FY 98 & & 3719 & COMPUTER FACLLITYTELECOMMUNICATTONS HUB & PHMC & N-Freels & 1978 & & 280 & $\mathrm{PCX}$ & 2 & & \\
\hline FY 98 & & 3722 & FABRICATION SHOPCONSTR LCTION SHOP & PHMC & N-Fuels & 1944 & & 463 & $\mathrm{PCx}$ & 0 & & \\
\hline FY 98 & & 3728 & $\begin{array}{l}\text { GEOTECHNICAL HIGH-BAY GEOTECHNICAL } \\
\text { HIGH-BAY }\end{array}$ & PHMC & & 1980 & & 297 & $\mathrm{PCx}$ & 0 & & \\
\hline FY 93 & & $3746 \mathrm{D}$ & $\begin{array}{l}\text { TECHNICAL SERVICE ANNEXTECHNICAL, SERVTCE } \\
\text { ANNEX }\end{array}$ & PHMC & N-Fuels & 1948 & & 117 & $\mathrm{PCX}$ & 0 & & \\
\hline FY 98 & & 3747 & $\begin{array}{l}\text { FUSYON MATERIAL IRRADUTKON TESTIFMTD } \\
\text { FACRITYFUSTON MATERIAL TRRADIATON } \\
\text { TESTFACIITY }\end{array}$ & PHMC & & & & & $\mathrm{PCx}$ & & & \\
\hline FY 98 & & $376 \mathrm{t}$ & $\begin{array}{l}\text { CONFERENCE TRALING BUILDINGCONFERENCE } \\
\text { TRAINING }\end{array}$ & PHMC & & & & & $\mathrm{PCx}$ & & & \\
\hline FY 98 & & 3771 & OFFICE BUILDING & PFMC & & & & & $\mathrm{PCx}$ & & & \\
\hline FY 98 & & 3772 & OFFICE BUILDING & PHMC & & & & & $\mathrm{PCX}$ & & & \\
\hline FY 98 & & 4166 & TRANSFER STORAGE BLILDING, FMEF & PHMC & & & & & $\overline{\mathrm{PCx}}$ & & & \\
\hline FY 98 & & $420 \mathrm{GOLFCLUB}$ & 420 GOLF CLLU ROAD, LACEY WA. & PHMC & & & & 116 & $\mathrm{PCx}$ & 0 & & \\
\hline FY 98 & & $46018 \mathrm{~B}$ & $\begin{array}{l}\text { CONTROL STRUCT. \& PROCESS SEWERSAMPLING } \\
\text { STATHONSEWER SAMPLING STATION }\end{array}$ & DYN & & & & 514 & $\mathrm{PCy}$ & & & \\
\hline FY 98 & & 4622 & METEROLOGY BUILING AND TOWER & PHMC & & & & & $\mathrm{PCx}$ & & & \\
\hline FY 98 & & $4717 \mathrm{~A}$ & CASK LOADING STATION (PART OF 4717) & PHMC & & & & & $\mathrm{PCy}$ & & & \\
\hline FY 98 & & $4718 \mathrm{TC}$ & OFFICE. TEMPORARY & PHMC & & & & & $\mathrm{PCx}$ & & & \\
\hline FY 98 & & $4732 \mathrm{~A}$ & WAREHOUSE WAREHOUSE & PHMC & ?FFTF & & & 1394 & $\mathrm{PCx}$ & & & \\
\hline FY 98 & & 4760 & CONSTRUCTION SHOP ELECTRICAL SHOP & PHMC & ?FFTF & & & 372 & $\mathrm{PCx}$ & & & \\
\hline FY 98 & & $4791 \mathrm{TC}$ & WAREHOUSE GENERAL STORAGE & BWHC & FFTF & & & 134 & $\overline{\mathrm{PCX}}$ & & & \\
\hline FY 98 & & 4814 & $\begin{array}{l}\text { SPECLAL TOOLS WAREHOLSE WAREHOUSE } \\
\text { BULDING }\end{array}$ & BWHC & FFTF & & & 465 & $\mathrm{PCx}$ & & & \\
\hline FY 98 & & 4831 & $\begin{array}{l}\text { LAYDOWN HAZARDOLIS STAGING FLAMMABLE } \\
\text { STORAGE BUILDENG }\end{array}$ & BWHC & FFTF & & & 137 & $\mathrm{PCx}$ & & & \\
\hline FY 98 & & G01WILLIAMS & $\begin{array}{l}\text { HAPO BUILDNG (601 } \\
\text { WILLIAMS, RICHLAND) OFFECE BUILDING }\end{array}$ & DYN & & & & & PCy & & & \\
\hline FY 98 & & 604 & YAKIMA BARRICADE, PATROLCHECKING STATION & PHMC & & & & 28 & $P C x$ & & & \\
\hline FY 98 & & $604 \mathrm{H}$ & $\begin{array}{l}\text { PATROL UTILTTY BULDINGPATROL LTILITY } \\
\text { BLTING }\end{array}$ & PHMC & & & & 2 & $\overline{P C x}$ & & & \\
\hline FY 98 & & 607 & BATCH PLANT LAB STORAGE - LESS THAN 90 DAY & PHMC & & & & 110 & $\mathrm{PCx}$ & & & \\
\hline $\mathrm{FY} 98$ & & 617 & BWR CORE STORAGE FACILITY & PHMC & & & & 1672 & $\mathrm{PCx}$ & & & \\
\hline
\end{tabular}




\begin{tabular}{|c|c|c|c|c|c|c|c|c|c|c|c|c|}
\hline $\begin{array}{l}\text { EVALV } \\
\text { SCHED }\end{array}$ & $\begin{array}{l}\text { PRIOITY } \\
\text { SCORE }\end{array}$ & IDENTIFIER & DESCRIPTION & CONTRA & PROJECT & $\begin{array}{l}\text { YEAR } \\
\text { BUILT }\end{array}$ & $\begin{array}{l}\text { YEAR } \\
\text { SURPLIS }\end{array}$ & $\begin{array}{l}\text { AREA SQ } \\
\text { METERS }\end{array}$ & $\begin{array}{c}\text { FERF.C } \\
\text { AT. }\end{array}$ & $\begin{array}{l}\text { TOTAL } \\
\text { OCCUP }\end{array}$ & $\begin{array}{l}\text { FACILITY } \\
\text { CONDITION } \\
\text { RATING }\end{array}$ & $\begin{array}{l}\text { EXISTING } \\
\text { ANALYSIS } \\
\text { RATING }\end{array}$ \\
\hline$F Y 98$ & & $6221 \mathrm{NA}$ & 6221N TOWER BUILDING & PHMC & & & & & $\mathrm{PCy}$ & & & \\
\hline FY 98 & & $6233 \mathrm{~A}$ & 5223 TOWER BUILDING & PHMC. & & & & & $\mathrm{PCy}$ & & & \\
\hline FY 98 & & $6224 \mathrm{~A}$ & 6224 TOWER BUILDING & PHMC & & & & & PCy & & & \\
\hline FY 98 & & $622 \mathrm{E}$ & STORAGE BLILDING STORAGE BLIIDING & PHMC & & & & 94 & $\mathrm{PCx}$ & & & \\
\hline FY 98 & & 623 & STORAGE BLILDING, GABLE MOLINTAIN & PHMC & & & & 17 & $\mathrm{PCx}$ & & & \\
\hline FY 98 & & 6291 & FUELING FACILTYFLELING FACIIITY & PHMC & & & & 26 & PCx & & & \\
\hline FY 98 & & $6652 \mathrm{~L}$ & $\begin{array}{l}\text { RATTLESNAKE MTN (FORMERL YFMERGENCY } \\
\text { CONTROL CNTR) ILNDERGROUND } \\
\text { EMERGENCYRELOCATION CENTER }\end{array}$ & PHMC: & & & & 669 & $\mathrm{POX}$ & & & \\
\hline FY 98 & & $6652 \mathrm{P}$ & GENERATOR BULDINGGENERATOR BUILDING & PHMC & & & & 84 & $\mathrm{PCx}$ & & & \\
\hline FY 98 & & $6652 \mathrm{R}$ & ACID STORAGE SHED & PHMC & & & & 4 & $\mathrm{PCx}$ & & & \\
\hline FY 98 & & $6652 \mathrm{~S}$ & SENTRY SHEDSENTRY SHED & PHMC & & & & 4 & $\mathrm{PCX}$ & & & \\
\hline FY 98 & & $6653 \mathrm{~A}$ & TEDF PUMP STATION \#3 & PHMC & & & & & $\mathrm{PCX}$ & & & \\
\hline FY 98 & & 712 & $\begin{array}{l}\text { RECORDS CENTER, PRLVTLNG ANDREPRODLCTION } \\
\text { PLANTRECORDS/PRINTINGMAIL }\end{array}$ & PHMC & & & & 1826 & $\mathrm{PC}$ & & & \\
\hline FY 98 & & $712 \mathbf{B}$ & IRM LITIGATION SUPPORT FACILITY & PHMC & & & & 172 & $\mathrm{PCx}$ & & & \\
\hline FY 98 & & 747 & $\begin{array}{l}\text { ENVIRONMENTAL HEALTH } \\
\text { SCIENCESBUWDINGENVIRONMENTAL HEALTH } \\
\text { SCIENCESBUTLDNG }\end{array}$ & PHMC & & & & 724 & $\mathrm{PCx}$ & & & \\
\hline FY 98 & & $747 \mathrm{~B}$ & $\begin{array}{l}\text { ENVIRONMENTAL HEALTH SCLENCESBUILDING } \\
\text { ANNEXENVIRONMENTAL HEALTH } \\
\text { SCIENCESANNEX }\end{array}$ & PHMC & & & & 231 & $\mathrm{PCx}$ & & & \\
\hline FY 98 & & MCC241AW & MOTOR CONTROL CENTER & LMHC & & & & & $\mathrm{PCx}$ & & & \\
\hline FY 98 & & MO023 & MORIE OFFICE Q 202AMOBILE OFFICE (202A) & BWHC & & & & 519 & $\mathrm{PCy}$ & & & \\
\hline$F Y 98$ & & MO046 & 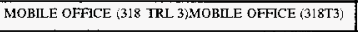 & DESH & & 1979 & & 515 & $\mathrm{PCy}$ & & & \\
\hline FY 98 & & MO114 & CHAKGE TRAILER G 24IBY & LMHC & & & & 28 & $\mathbf{P C x}$ & & & \\
\hline FY 98 & & MO286 & MOBILE OFFICE $Q 2727$ MOBLE OFFCE & DYN & & & & 1030 & $\mathrm{PCy}$ & & & \\
\hline FY 98 & & MO293 & MOBILE OFFICE $Q$ 1725K (SPENT FUEL) & DESH & $1.00 \mathrm{~K}$ & & & 859 & $\mathrm{PCy}$ & & & \\
\hline $\mathrm{FY} 98$ & & MO295 & TRAILER & LMHC & & & & 71 & $\mathrm{PCx}$ & & & \\
\hline FY 98 & & M0305 & MOBLE OFFCE 325 T. IMOBILE OFFCE (325T) & BWHC & & & & & PCy & & & \\
\hline FY 98 & & M0319 & CHANGE TRAILER (TANK FARMS) 241BX & LMHC & & & & 28 & PCx & & & \\
\hline FY 98 & & M0320 & WOMENS CHANGE ROOM TRAILER $9242 S$ & LMHC & & & & 28 & PCX & & & \\
\hline FY 98 & & M0333 & STORAGE TRARLER $\Theta 2$ W TANK FARMSU-12 & LMHC & & & & 28 & $\mathrm{PCx}$ & & & \\
\hline FY 98 & & MO363 & MOBILE OFFICE $Q 24 I S X$ TANK FARM & LMHC & & & & 18 & $\mathrm{PCx}$ & & & \\
\hline $\mathrm{FY} 98$ & & M0404 & MOBLE OFFICE $@$ I153MOBILE OFFICE $(1166)$ & DYN & & & & 687 & PCy & & & \\
\hline FY 98 & & M0405 & MOBLE OFFICE @ PUREXMOBILE OFFICE - PUREX & BWHC & & & & 1288 & PCy & & & \\
\hline FY 98 & & MO409 & $\begin{array}{l}\text { A.NALYTICAL LAB TRAILER @ PUREXMOBIIE } \\
\text { OFFICE - PUREX }\end{array}$ & BWHC & & & & 343 & PCy & & & \\
\hline
\end{tabular}


HNF-SD-GN-ER-507 REV 0

\begin{tabular}{|c|c|c|c|c|c|c|c|c|c|c|c|c|c|c|c|c|c|c|c|c|c|c|c|c|}
\hline 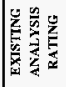 & & & & & & & & & & & & & & & & & & & & & & & & \\
\hline${ }_{0}$ & & & & & & & & & & & & & & & & & & & & & & & & \\
\hline 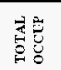 & & & & & & & & & & & & & & & & & & & & & $=$ & & & \\
\hline 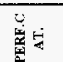 & 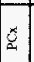 & 0 & 0 & 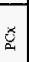 & $Q$ & 8 & 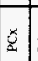 & 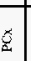 & 0 & 0 & 0 & 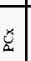 & 0 & $\varrho$ & 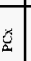 & ర్ర & 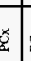 & $\hat{\varepsilon}$ & 8 & $\underline{Z}$ & 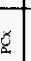 & 0 & 8 & 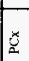 \\
\hline 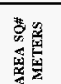 & 8 & $a$ & $a$ & $a$ & $\div$ & 号 & i & 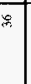 & i & 它 & 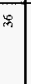 & is & 品 & 品 & 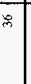 & $\bar{s}$ & 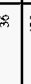 & 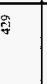 & 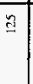 & & & & $\because$ & $=$ \\
\hline 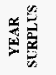 & & & & & & & & & & & & & & & & & & & & & & & & \\
\hline 要恶 & & & & & & & & & & & & & & & & & & & & & & & & \\
\hline $\begin{array}{l}\bar{U} \\
\bar{z} \\
\bar{z}\end{array}$ & & & & & & & & & & & & & 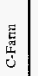 & & & & & & & & & & & \\
\hline$\frac{5}{8}$ & 牙 & 离 & 弯 & 畜 & 语 & 楒 & 遷 & 裉 & 莖 & 볼 & 員 & 总 & 容 & 䁧 & $\frac{ \pm}{3}$ & E & $\frac{\underline{x}}{\Sigma}$ & z. & 足 & $\sum_{\underline{\Sigma}}$ & 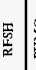 & 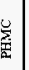 & 站 & 竞 \\
\hline 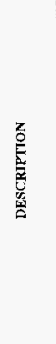 & 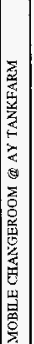 & 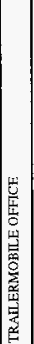 & 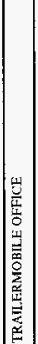 & 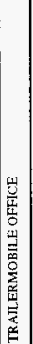 & 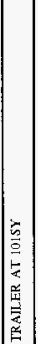 & 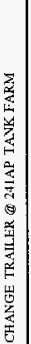 & 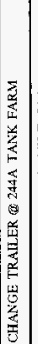 & 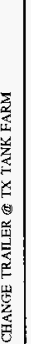 & 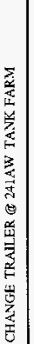 & 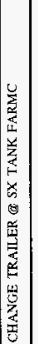 & 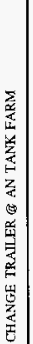 & 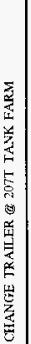 & 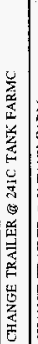 & 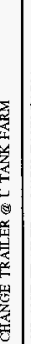 & 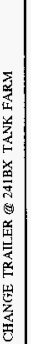 & 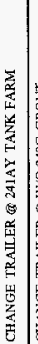 & 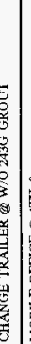 & 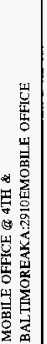 & 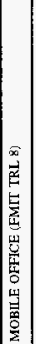 & 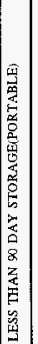 & 童 & 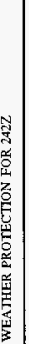 & 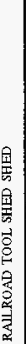 & 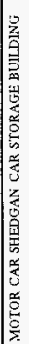 \\
\hline 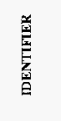 & $\begin{array}{l}\frac{a}{0} \\
\frac{a}{2} \\
\frac{0}{2}\end{array}$ & $\frac{9}{\frac{9}{2}}$ & 縍 & $\overline{\frac{3}{3}}$ & $\mid$\begin{tabular}{|l}
$\overrightarrow{\frac{\sigma}{2}}$ \\
$\frac{\partial}{2}$
\end{tabular} & 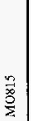 & 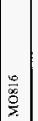 & 恿 & $\begin{array}{l}\frac{x}{2} \\
\frac{x}{2} \\
\end{array}$ & $\mid \begin{array}{l}\frac{a}{p} \\
\frac{\partial}{2} \\
\frac{2}{2}\end{array}$ & $\begin{array}{l}\frac{7}{0} \\
\frac{c}{2}\end{array}$ & $\frac{\pi}{\frac{\pi}{2}}$ & $\frac{g}{\frac{g}{2}}$ & 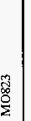 & $\begin{array}{l}\text { 茄 } \\
\frac{8}{2}\end{array}$ & $\begin{array}{l}0 \\
\frac{5}{3} \\
\frac{3}{2}\end{array}$ & 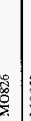 & 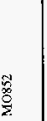 & 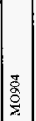 & $\frac{2}{2}$ & 管 & 品 & 菏 & $\underset{x}{\infty}$ \\
\hline 夏哭 & & & & & & & & & & & & & & & & & & & & & & & & \\
\hline 量 & $\frac{2}{2}$ & 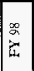 & $\begin{array}{l}\infty \\
\Sigma \\
亡\end{array}$ & $\mid \begin{array}{l}\approx \\
\Sigma \\
\Sigma\end{array}$ & $\begin{array}{l}x \\
\dot{x} \\
\dot{L}\end{array}$ & 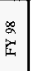 & 总 & z & $\begin{array}{l}\text { ga } \\
\text { 至 } \\
\end{array}$ & $\mid \begin{array}{l}\mathrm{g} \\
\mathrm{I}\end{array}$ & 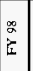 & $\begin{array}{l}z \\
z \\
z\end{array}$ & \begin{tabular}{|l|} 
\\
0 \\
0 \\
\end{tabular} & 梁 & $\begin{array}{l}8 \\
2 \\
5\end{array}$ & 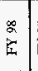 & $\begin{array}{l}\text { 年 } \\
\text { 庄 }\end{array}$ & 总 & \begin{tabular}{|l|}
$\infty$ \\
$\tilde{L}$ \\
$\tilde{L}$
\end{tabular} & $\begin{array}{l}\infty \\
2 \\
\end{array}$ & 蛋 & 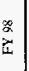 & $\vec{F}$ & $\underset{\check{I}}{\infty}$ \\
\hline
\end{tabular}

